Supporting Information

\title{
Enantioselective synthesis of pyrrole fused piperazine and piperazinone derivatives via Ir-catalyzed asymmetric allylic amination
}

Chun-Xiang Zhuo, Xiao Zhang, and Shu-Li You*

State Key Laboratory of Organometallic Chemistry

Shanghai Institute of Organic Chemistry, Chinese Academy of Sciences 345 Lingling Lu, Shanghai 200032, China

E-mail: slyou@sioc.ac.cn

\section{Table of Contents}

Optimization of the reaction conditions

S2

General methods

S3

Experimental details and characterization data

S4-19

X-Ray crystal structure of 3a

S20-21

Copies of NMR Spectra

S22-39

Copies of HPLC Chromatographs

S40-51 
Table S1: Optimization of the reaction conditions ${ }^{a}$

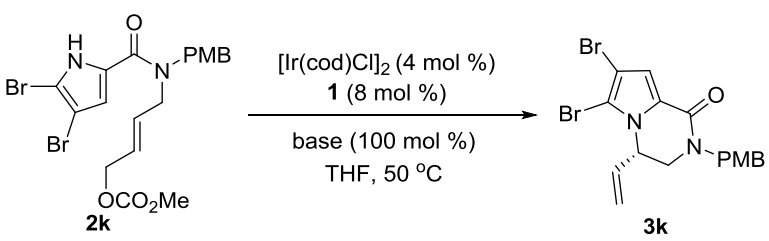

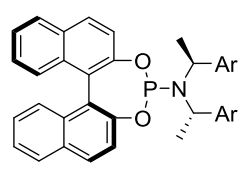

$\left(S, S, S_{a}\right)-1 \mathbf{a}: \mathrm{Ar}=\mathrm{Ph}$

$\left(S, S, S_{2}\right)-1 \mathbf{b}: \mathrm{Ar}=2-\mathrm{MeO}-\mathrm{C}_{6} \mathrm{H}_{4}$

$\left(S, S, S_{a}\right)-1 \mathrm{c}:$ Ar $=2$-naphthyl

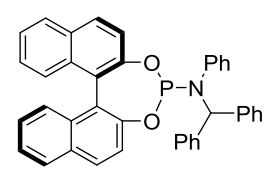

$\left(R_{\mathrm{a}}\right)-\mathbf{1 d}$

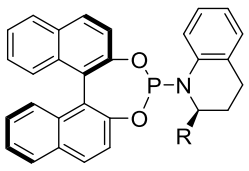

$\left(R, R_{a}\right)-1 \mathrm{e}: \mathrm{R}=\mathrm{Me}$ $\left(R, R_{a}\right)-1 \mathbf{g}: \mathrm{R}=\mathrm{Et}$ $\left(R, R_{a}\right)-1 \mathrm{~h}: \mathrm{R}=\mathrm{Ph}$

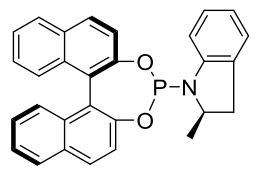

$\left(R, R_{a}\right)-1 \mathrm{i}$

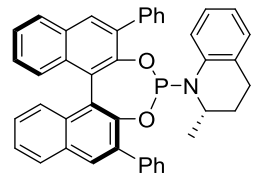

$\left(S, S_{a}\right)-\mathbf{1 f}$

\begin{tabular}{|c|c|c|c|c|c|}
\hline entry & 1 & base & time $(\mathrm{h})$ & yield $(\%)^{b}$ & ee $(\%)^{c}$ \\
\hline 1 & 1a & $\mathrm{Cs}_{2} \mathrm{CO}_{3}$ & 64 & 80 & 63 \\
\hline $2^{d}$ & $1 \mathrm{~b}$ & $\mathrm{Cs}_{2} \mathrm{CO}_{3}$ & 65 & 86 & 67 \\
\hline 3 & $1 c$ & $\mathrm{Cs}_{2} \mathrm{CO}_{3}$ & 64 & 76 & 61 \\
\hline 4 & 1d & $\mathrm{Cs}_{2} \mathrm{CO}_{3}$ & 64 & 98 & 83 \\
\hline 5 & 1e & $\mathrm{Cs}_{2} \mathrm{CO}_{3}$ & 64 & 94 & 89 \\
\hline 6 & $1 \mathrm{~g}$ & $\mathrm{Cs}_{2} \mathrm{CO}_{3}$ & 63 & 81 & 87 \\
\hline 7 & $1 \mathrm{~h}$ & $\mathrm{Cs}_{2} \mathrm{CO}_{3}$ & 63 & 80 & 80 \\
\hline 8 & $1 \mathrm{i}$ & $\mathrm{Cs}_{2} \mathrm{CO}_{3}$ & 63 & 82 & 57 \\
\hline 9 & 1f & $\mathrm{Cs}_{2} \mathrm{CO}_{3}$ & 63 & 95 & -91 \\
\hline 10 & $1 e$ & $\mathrm{~K}_{3} \mathrm{PO}_{4}$ & 64 & 84 & 92 \\
\hline 11 & $1 e$ & DBU & 64 & 99 & 95 \\
\hline 12 & $1 e$ & dabco & 60 & 85 & 92 \\
\hline $13^{e}$ & $1 \mathrm{e}$ & DBU & 70 & 96 & 96 \\
\hline
\end{tabular}

${ }^{a}$ Reaction conditions: $4 \mathrm{~mol} \%$ of $[\operatorname{Ir}(\operatorname{cod}) \mathrm{Cl}]_{2}, 8 \mathrm{~mol} \%$ of $\mathbf{L}, 0.05 \mathrm{mmol}$ of $\mathbf{2 k}$ and base in $0.5 \mathrm{~mL}$ of THF. ${ }^{b}$ Isolated yield. ${ }^{c}$ Determined by HPLC analysis. ${ }^{d}$ Reaction conditions: 2 mol \% of $[\operatorname{Ir}(\operatorname{cod}) \mathrm{Cl}]_{2}$, $4 \mathrm{~mol} \%$ of $\mathbf{1 b}, 0.2 \mathrm{mmol}$ of $\mathbf{2} \mathbf{k}$ and $\mathrm{Cs}_{2} \mathrm{CO}_{3}$ in $2.0 \mathrm{~mL}$ of THF. ${ }^{e}$ Reaction conditions: 2 mol $\%$ of $[\operatorname{Ir}(\operatorname{cod}) \mathrm{Cl}]_{2}, 4 \mathrm{~mol} \%$ of $\mathbf{1 e}, 0.2 \mathrm{mmol}$ of $\mathbf{2 k}$ and DBU in $2.0 \mathrm{~mL}$ of THF. 
General methods. Unless stated otherwise, all reactions were carried out in flame-dried glassware under a dry argon atmosphere. All solvents were purified and dried according to standard methods prior to use.

${ }^{1} \mathrm{H}$ and ${ }^{13} \mathrm{C}$ NMR spectra were recorded on a Varian instrument $(300,400 \mathrm{MHz}$ and 75, $100 \mathrm{MHz}$, respectively) or an Agilent instrument (400, $600 \mathrm{MHz}$ and 100, 150 $\mathrm{MHz}$, respectively) and internally referenced to tetramethylsilane signal or residual protio solvent signals. ${ }^{19} \mathrm{~F}$ NMR spectra were recorded on a Varian instrument (282 $\mathrm{MHz}$ and $376 \mathrm{MHz}$, respectively) and referenced relative to $\mathrm{CFCl}_{3}$. Data for ${ }^{1} \mathrm{H} \mathrm{NMR}$ are recorded as follows: chemical shift $(\delta, \mathrm{ppm})$, multiplicity $(\mathrm{s}=$ singlet, $\mathrm{d}=$ doublet, $\mathrm{t}=$ triplet, $\mathrm{m}=$ multiplet or unresolved, $\mathrm{br}=$ broad singlet, coupling constant $(\mathrm{s})$ in $\mathrm{Hz}$, integration). Data for ${ }^{13} \mathrm{C}$ NMR are reported in terms of chemical shift ( $\delta$, ppm). The crystal structure was determined with $\mathrm{Cu} \mathrm{K} \alpha$ radiation by Bruker APEX DUO single crystal X-ray diffractometer.

The phosphoramidite ligands, ${ }^{1}$ 2-substituted 5-pyrrolyl methanamines $\mathbf{S 1}^{2}$ and (E)-4-bromo-but-2-enyl methyl ester $^{3}$ were prepared according to the reported procedures. 
Scheme S1: General procedure for the synthesis of substituted allylic carbonates 2a-b and 2e-h.<smiles>[R17]NCc1ccc(C([R])=O)[nH]1</smiles>

s1

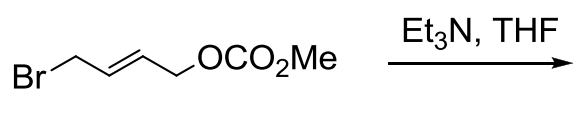

(2)

To a solution of the 2-substituted 5-pyrrolyl methanamines S1 (2 mmol, 1.0 equiv) and $\mathrm{Et}_{3} \mathrm{~N}(0.4 \mathrm{~mL}, 2.4 \mathrm{mmol})$ in dry $\mathrm{THF}(25 \mathrm{~mL})$, carbonic acid (E)-4-bromo-but-2-enyl methyl ester $(541 \mathrm{mg}, 2.6 \mathrm{mmol})$ was added at $0{ }^{\circ} \mathrm{C}$. The ice bath was removed and the reaction mixture was stirred at $\mathrm{rt}$ overnight. After the reaction was complete (monitored by TLC), the crude reaction mixture was filtrated with celite and washed with EtOAc. The solvents were removed under reduced pressure. Then the residue was purified by silica gel column chromatography (PE/EA $=4 / 1)$ to afford the desired product 2 .

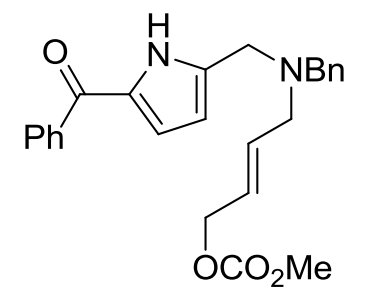

2a. Viscous yellow oil, 93\% yield. ${ }^{1} \mathrm{H}$ NMR $\left(300 \mathrm{MHz}, \mathrm{CDCl}_{3}\right) \delta 10.50$ (br s, 1H), $7.91(\mathrm{~d}, J=7.5 \mathrm{~Hz}, 2 \mathrm{H}), 7.57-7.47(\mathrm{~m}, 3 \mathrm{H}), 7.35-7.25(\mathrm{~m}, 5 \mathrm{H}), 6.85(\mathrm{t}, J=3.0$ $\mathrm{Hz}, 1 \mathrm{H}), 6.22(\mathrm{t}, J=2.7 \mathrm{~Hz}, 1 \mathrm{H}), 5.92-5.85(\mathrm{~m}, 1 \mathrm{H}), 5.78-5.71(\mathrm{~m}, 1 \mathrm{H}), 4.59$ (d, $J=$ $5.7 \mathrm{~Hz}, 2 \mathrm{H}), 3.77(\mathrm{~s}, 3 \mathrm{H}), 3.70(\mathrm{~s}, 2 \mathrm{H}), 3.59(\mathrm{~s}, 2 \mathrm{H}), 3.10(\mathrm{~d}, J=5.7 \mathrm{~Hz}, 2 \mathrm{H}) .{ }^{13} \mathrm{C}$ NMR $\left(75 \mathrm{MHz}, \mathrm{CDCl}_{3}\right) \delta 184.0,155.3,138.3,138.2,132.6,131.3,130.2,128.7$, $128.5,128.1,128.0,126.8,126.4,120.4,110.1,67.5,57.7,54.8,54.5,50.0$. IR (thin film): $v_{\max }\left(\mathrm{cm}^{-1}\right)=3265,3061,3027,2956,2925,1746,1605,1571,1444,1259,943$, 791; HRMS (ESI) calcd for $\mathrm{C}_{25} \mathrm{H}_{27} \mathrm{~N}_{2} \mathrm{O}_{4}[\mathrm{M}+\mathrm{H}]^{+}$: 419.1965 . Found: 419.1973. 


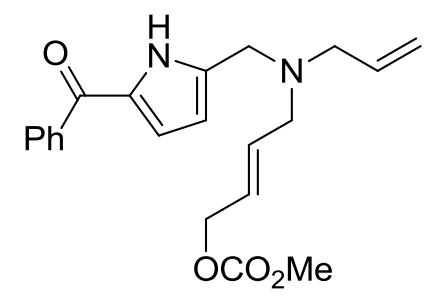

2b. Brown solid, m.p. $=48.2-50.5{ }^{\circ} \mathrm{C}, 72 \%$ yield. ${ }^{1} \mathrm{H}$ NMR $\left(300 \mathrm{MHz}, \mathrm{CDCl}_{3}\right) \delta$ 10.37 (br s, 1H), $7.85(\mathrm{~d}, J=8.1 \mathrm{~Hz}, 2 \mathrm{H}), 7.49-7.40(\mathrm{~m}, 3 \mathrm{H}), 6.77(\mathrm{t}, J=3.3 \mathrm{~Hz}, 1 \mathrm{H})$, $6.11(\mathrm{t}, J=3.0 \mathrm{~Hz}, 1 \mathrm{H}), 5.86-5.64(\mathrm{~m}, 3 \mathrm{H}), 5.13(\mathrm{~d}, J=15.3 \mathrm{~Hz}, 1 \mathrm{H}), 5.10(\mathrm{~d}, J=8.7$ $\mathrm{Hz}, 1 \mathrm{H}), 4.54(\mathrm{~d}, J=5.7 \mathrm{~Hz}, 2 \mathrm{H}), 3.72(\mathrm{~s}, 3 \mathrm{H}), 3.62(\mathrm{~s}, 2 \mathrm{H}), 3.05(\mathrm{~d}, J=5.1 \mathrm{~Hz}, 2 \mathrm{H})$, $3.04(\mathrm{~d}, J=5.4 \mathrm{~Hz}, 2 \mathrm{H}) .{ }^{13} \mathrm{C} \mathrm{NMR}\left(75 \mathrm{MHz}, \mathrm{CDCl}_{3}\right) \delta 184.0,155.2,138.2,138.0$, $134.7,132.3,131.3,130.1,128.6,127.9,126.4,120.3,117.8,109.9,67.5,56.4,54.7$, 54.4, 49.7. IR (thin film): $v_{\max }\left(\mathrm{cm}^{-1}\right)=3267,3075,2953,2926,1750,1598,1569$, 1479, 1372, 1178, 972, 719; HRMS (ESI) calcd for $\mathrm{C}_{21} \mathrm{H}_{25} \mathrm{~N}_{2} \mathrm{O}_{4}[\mathrm{M}+\mathrm{H}]^{+}$: 369.1809 . Found: 369.1816.

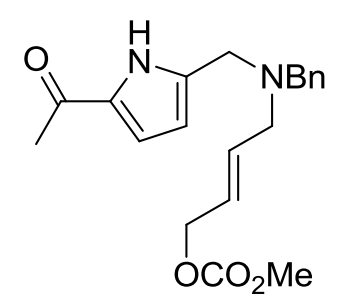

2e. Brown solid, m.p. $=48.3-50.4{ }^{\circ} \mathrm{C}, 52 \%$ yield. ${ }^{1} \mathrm{H} \mathrm{NMR}\left(300 \mathrm{MHz}, \mathrm{CDCl}_{3}\right) \delta$ $9.53($ br s, $1 \mathrm{H}), 7.32-7.21(\mathrm{~m}, 5 \mathrm{H}), 6.82(\mathrm{t}, J=3.0 \mathrm{~Hz}, 1 \mathrm{H}), 6.09(\mathrm{t}, J=3.9 \mathrm{~Hz}, 1 \mathrm{H})$, 5.91-5.82 (m, 1H), 5.79-5.70 (m, 1H), $4.60(\mathrm{~d}, J=6.0 \mathrm{~Hz}, 2 \mathrm{H}), 3.78(\mathrm{~s}, 3 \mathrm{H}), 3.57(\mathrm{~s}$, 2H), 3.55 (s, 2H), 3.06 (d, $J=6.9 \mathrm{~Hz}, 2 \mathrm{H}), 2.39$ (s, 3H). ${ }^{13} \mathrm{C} \mathrm{NMR}\left(75 \mathrm{MHz}, \mathrm{CDCl}_{3}\right.$ ) $\delta 187.3,155.5,138.2,137.2,132.6,131.5,128.8,128.3,127.1,126.8,117.3,109.7$, 67.7, 58.0, 55.0, 54.7, 50.1, 25.1. IR (thin film): $v_{\max }\left(\mathrm{cm}^{-1}\right)=3300,3028,2956,2927$, 1745, 1680, 1597, 1479, 1261, 1209, 947, 736; HRMS (ESI) calcd for $\mathrm{C}_{20} \mathrm{H}_{26} \mathrm{~N}_{2} \mathrm{O}_{4}$ $[\mathrm{M}+\mathrm{H}]^{+}:$357. 1809. Found: 357.1796. 


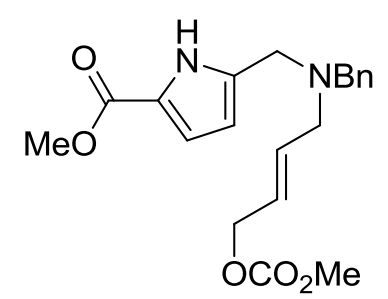

2f. Brown solid, m.p. $=53.9-56.2{ }^{\circ} \mathrm{C}, 66 \%$ yield. ${ }^{1} \mathrm{H}$ NMR $\left(300 \mathrm{MHz}, \mathrm{CDCl}_{3}\right) \delta$ 9.42 (br s, 1H), 7.32-7.31 (m, 5H), $6.84(\mathrm{t}, J=3.3 \mathrm{~Hz}, 1 \mathrm{H}), 6.08(\mathrm{t}, J=3.3 \mathrm{~Hz}, 1 \mathrm{H})$, 5.92-5.85 (m, 1H), 5.80-5.68 (m, 1H), $4.61(\mathrm{~d}, J=5.7 \mathrm{~Hz}, 1 \mathrm{H}), 3.84(\mathrm{~s}, 3 \mathrm{H}), 3.78(\mathrm{~s}$, $3 \mathrm{H}), 3.58(\mathrm{~s}, 2 \mathrm{H}), 3.56(\mathrm{~s}, 2 \mathrm{H}), 3.07(\mathrm{~d}, J=5.7 \mathrm{~Hz}, 2 \mathrm{H}) .{ }^{13} \mathrm{C} \mathrm{NMR}\left(75 \mathrm{MHz}, \mathrm{CDCl}_{3}\right)$ $\delta 161.4,155.5,138.3,135.1,132.6,128.7,128.2,127.1,126.7,121.6,115.6,109.4$, 67.7, 57.8, 54.8, 54.7, 51.2, 50.0. IR (thin film): $v_{\max }\left(\mathrm{cm}^{-1}\right)=3309,3033,2949,2917$, $1748,1677,1482,1453,1260,1215,758,699$; HRMS (ESI) calcd for $\mathrm{C}_{20} \mathrm{H}_{25} \mathrm{~N}_{2} \mathrm{O}_{5}$ $[\mathrm{M}+\mathrm{H}]^{+}:$373.1758. Found: 373.1764 .

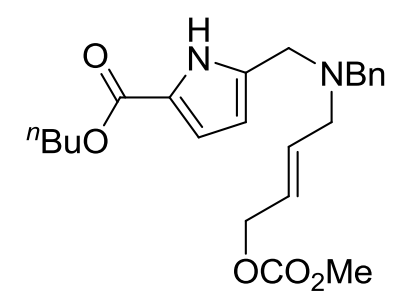

2g. Brown solid, m.p. $=39.2-41.2{ }^{\circ} \mathrm{C}, 53 \%$ yield. ${ }^{1} \mathrm{H} \mathrm{NMR}\left(300 \mathrm{MHz}, \mathrm{CDCl}_{3}\right) \delta$ $9.52(\mathrm{~s}, 1 \mathrm{H}), 7.30-7.21(\mathrm{~m}, 5 \mathrm{H}), 6.83(\mathrm{~m}, 1 \mathrm{H}), 6.07(\mathrm{~m}, 1 \mathrm{H}), 5.90-5.83(\mathrm{~m}, 1 \mathrm{H})$, 5.76-5.67 (m, 1H), $4.59(\mathrm{~d}, J=6.0 \mathrm{~Hz}, 1 \mathrm{H}), 4.24(\mathrm{t}, J=6.9 \mathrm{~Hz}, 2 \mathrm{H}), 3.75(\mathrm{~s}, 3 \mathrm{H})$, $3.57(\mathrm{~s}, 2 \mathrm{H}), 3.54(\mathrm{~s}, 2 \mathrm{H}), 3.06(\mathrm{~d}, J=5.7 \mathrm{~Hz}, 2 \mathrm{H}), 1.72-1.67(\mathrm{~m}, 2 \mathrm{H}), 1.45-1.43(\mathrm{~m}$, $2 \mathrm{H}), 0.96(\mathrm{t}, J=7.8 \mathrm{~Hz}, 3 \mathrm{H}) .{ }^{13} \mathrm{C} \mathrm{NMR}\left(75 \mathrm{MHz}, \mathrm{CDCl}_{3}\right) \delta 161.1,155.4,138.3$, 134.9, 132.6, 128.6, 128.2, 127.0, 126.6, 121.9, 115.4, 109.3, 67.6, 63.8, 57.7, 54.7, 54.5, 50.0, 30.7, 19.0, 13.6. IR (thin film): $v_{\max }\left(\mathrm{cm}^{-1}\right)=3297,2958,2920,2877,1745$, 1671, 1482, 1441, 1261, 1216, 737, 698; HRMS (ESI) calcd for $\mathrm{C}_{23} \mathrm{H}_{31} \mathrm{~N}_{2} \mathrm{O}_{5}[\mathrm{M}+\mathrm{H}]^{+}$: 415.2227. Found: 415.2215. 


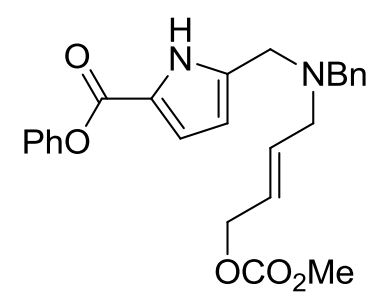

2h. Brown solid, m.p. $=77.8-80.1{ }^{\circ} \mathrm{C}, 84 \%$ yield. ${ }^{1} \mathrm{H}$ NMR $\left(300 \mathrm{MHz}, \mathrm{CDCl}_{3}\right) \delta$ 9.64 (br s, 1H), 7.41-7.31 (m, 2H), 7.29-7.23 (m, 4H), 7.19-7.17 (m, 4H), 7.05 (t, $J=$ $3.0 \mathrm{~Hz}, 1 \mathrm{H}), 6.15(\mathrm{t}, J=3.0 \mathrm{~Hz}, 1 \mathrm{H}), 5.89-5.69(\mathrm{~m}, 2 \mathrm{H}), 4.59(\mathrm{~d}, J=5.4 \mathrm{~Hz}, 2 \mathrm{H})$, $3.74(\mathrm{~s}, 3 \mathrm{H}), 3.54(\mathrm{~s}, 2 \mathrm{H}), 3.53(\mathrm{~s}, 2 \mathrm{H}), 3.04(\mathrm{~d}, J=6.0 \mathrm{~Hz}, 2 \mathrm{H}) .{ }^{13} \mathrm{C}$ NMR $(75 \mathrm{MHz}$, $\left.\mathrm{CDCl}_{3}\right) \delta 159.3,155.4,150.5,138.3,136.6,132.6,129.2,128.7,128.3,127.1,126.6$, $125.5,121.7,120.8,117.3,109.8,67.7,57.9,54.8,54.6,50.0$. IR (thin film): $v_{\max }$ $\left(\mathrm{cm}^{-1}\right)=3301,3029,2956,2822,1745,1680,1478,1444,1262,1196,736,691 ;$ HRMS (ESI) calcd for $\mathrm{C}_{25} \mathrm{H}_{27} \mathrm{~N}_{2} \mathrm{O}_{5}[\mathrm{M}+\mathrm{H}]^{+}:$435.1914. Found: 435.1896.

Scheme S2: General procedure for the synthesis of substituted allylic carbonates 2c-2d.<smiles>[X]Cc1ccc(C(=O)c2ccccc2)[nH]1</smiles><smiles></smiles>

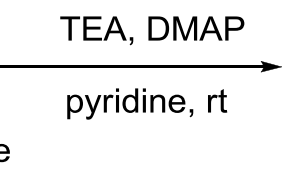<smiles>COCC=CCCc1ccc(C(=O)c2ccccc2)[nH]1</smiles>

To a solution of the substituted allylic alcohol S2 (1.45 mmol, 1.0 equiv) and $\mathrm{Et}_{3} \mathrm{~N}(4 \mathrm{~mL})$ in pyridine $(8 \mathrm{~mL})$, compound $\mathbf{S 3}(385 \mathrm{mg}, 2.0 \mathrm{mmol})$ and catalytic amount of DMAP $(20 \mathrm{mg})$ was added at $\mathrm{rt}$. The reaction mixture was stirred at $\mathrm{rt}$ overnight. After the reaction was complete (monitored by TLC), the crude reaction mixture was diluted with EtOAc $(20 \mathrm{~mL})$ and washed with water $(10 \mathrm{~mL})$ and saturated $\mathrm{CuSO}_{4}$ aqueous solution $(20 \mathrm{~mL}$ x 3). The organic layers were washed with brine, dried over $\mathrm{Na}_{2} \mathrm{SO}_{4}$. The solvents were removed under reduced pressure. Then the residue was purified by silica gel column chromatography $(\mathrm{PE} / \mathrm{EA}=6 / 1)$ to afford the desired product $\mathbf{2 c} \& \mathbf{2 d}$. 


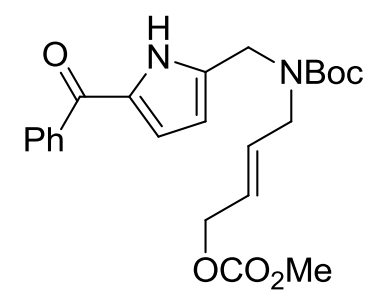

2c. Yellow oil, $72 \%$ yield. ${ }^{1} \mathrm{H}$ NMR $\left(300 \mathrm{MHz}, \mathrm{CDCl}_{3}\right) \delta 10.51$ (br s, $1 \mathrm{H}$ ), 7.83 (d, $J=7.2 \mathrm{~Hz}, 2 \mathrm{H}$ ), 7.44-7.41 (m, 3H), 6.74 (br s, 1H), 6.12 (br s, 1H), 5.63-5.62 (m, 2H), $4.51(\mathrm{~d}, J=4.5 \mathrm{~Hz}, 2 \mathrm{H}), 4.38(\mathrm{~s}, 2 \mathrm{H}), 3.85-3.79(\mathrm{~m}, 2 \mathrm{H}), 3.70(\mathrm{~s}, 3 \mathrm{H}), 1.45$ (br s, 9H). ${ }^{13} \mathrm{C}$ NMR $\left(75 \mathrm{MHz}, \mathrm{CDCl}_{3}\right) \delta 184.3,155.8,155.3,154.7,138.2,137.0,131.5$, 130.7, 130.4, 128.7, 128.1, 125.8, 125.6, 119.8, 110.0, 80.7, 80.4, 67.2, 54.5, 48.2, 47.9, 42.9, 28.1. IR (thin film): $v_{\max }\left(\mathrm{cm}^{-1}\right)=3259,2960,2924,1748,1694,1606$, 1571, 1258, 1229, 1160, 790; HRMS (ESI) calcd for $\mathrm{C}_{23} \mathrm{H}_{29} \mathrm{~N}_{2} \mathrm{O}_{6}[\mathrm{M}+\mathrm{H}]^{+}: 429.2020$. Found: 429.2028.

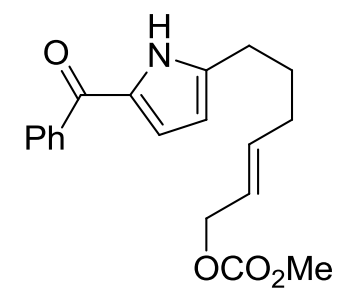

2d. Brown solid, m.p. $=67.9-70.2{ }^{\circ} \mathrm{C}, 78 \%$ yield. ${ }^{1} \mathrm{H}$ NMR $\left(300 \mathrm{MHz}, \mathrm{CDCl}_{3}\right) \delta$ 11.13 (br s, 1H), 7.92-7.88 (m, 2H), 7.57-7.45 (m, 3H), 6.83-6.82 (m, 1H), 6.07-6.05 (m, 1H), 5.80-5.70 (m, 1H), 5.60-5.51 (m, 1H), $4.52(\mathrm{~d}, J=5.7 \mathrm{~Hz}, 2 \mathrm{H}), 3.76(\mathrm{~s}, 3 \mathrm{H})$, $2.74(\mathrm{t}, J=7.5 \mathrm{~Hz}, 2 \mathrm{H}), 2.11-2.03(\mathrm{~m}, 2 \mathrm{H}), 1.83-1.76(\mathrm{~m}, 2 \mathrm{H}) .{ }^{13} \mathrm{C} \mathrm{NMR}(75 \mathrm{MHz}$, $\left.\mathrm{CDCl}_{3}\right) \delta 184.0,155.5,142.3,138.6,136.0,131.3,129.9,128.8,128.0,123.7,121.4$, 109.1, 68.3, 54.5, 31.5, 28.2, 27.1. IR (thin film): $v_{\max }\left(\mathrm{cm}^{-1}\right)=3273,2957,2923$, $1737,1597,1569,1496,1248,1232,793,696$; HRMS (ESI) calcd for $\mathrm{C}_{19} \mathrm{H}_{22} \mathrm{NO}_{4}$ $[\mathrm{M}+\mathrm{H}]^{+}: 328.1543$. Found: 328.1558.

Scheme S3: General procedure for the synthesis of substituted allylic carbonates 2i-2l. 


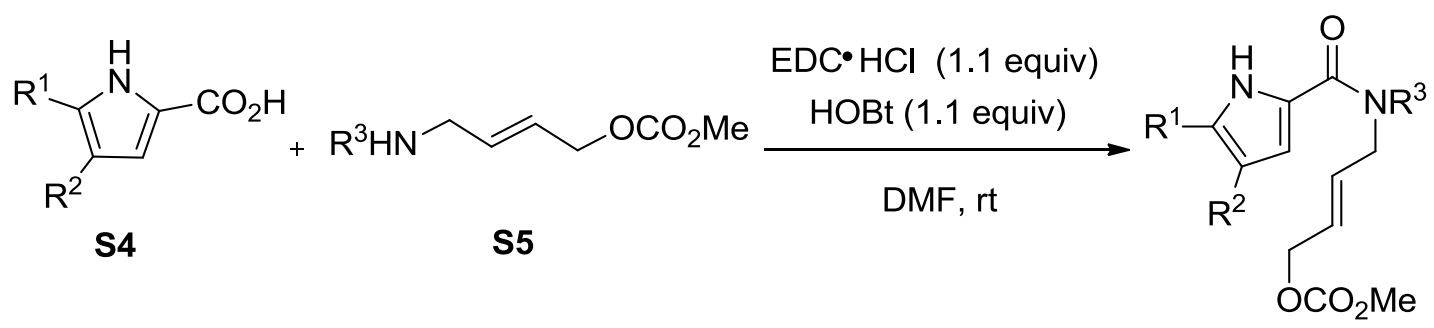

To a solution of the substituted $1 H$-pyrrole-2-carboxylic acid $\mathbf{S 4}$ (2 mmol, 1.0 equiv) in DMF (10 mL), 1-hydroxybenzotrizole (HOBt) (297 mg, $2.2 \mathrm{mmol})$ and $\mathrm{N}$-(3-dimethylaminopropyl)-N'-ethylcarbodiimide hydrochloride (EDC $\bullet \mathrm{HCl})(422$ mg, $2.2 \mathrm{mmol}$ ) were added. This mixture was stirred for 30 minutes at $\mathrm{rt}$, then compound S5 (2 mmol, 1.0 equiv) was added. After the reaction was complete (monitored by TLC), the crude reaction mixture was diluted with EtOAc $(20 \mathrm{~mL})$ and washed with water $(10 \mathrm{~mL} \times 3)$ and brine $(15 \mathrm{~mL})$. The organic layers were combined and dried over $\mathrm{Na}_{2} \mathrm{SO}_{4}$. The solvents were removed under reduced pressure. Then the residue was purified by silica gel column chromatography $(\mathrm{PE} / \mathrm{EA}=5 / 1)$ to afford the desired product $\mathbf{2 i} \& \mathbf{2 l}$.<smiles>COC/C=C/CN(Cc1ccccc1)C(=O)c1ccc[nH]1</smiles>

2i. White solid, m.p. $=58.9-61.2{ }^{\circ} \mathrm{C}, 61 \%$ yield. ${ }^{1} \mathrm{H}$ NMR $(400 \mathrm{MHz}, \mathrm{D} 6-\mathrm{DMSO}$, $\left.80{ }^{\circ} \mathrm{C}\right) \delta 11.22(\mathrm{br} \mathrm{s}, 1 \mathrm{H}), 7.31-7.24(\mathrm{~m}, 5 \mathrm{H}), 6.89-6.88(\mathrm{~m}, 1 \mathrm{H}), 6.40-6.39(\mathrm{~m}, 1 \mathrm{H})$, 6.07-6.06 (m, 1H), 5.87-5.81 (m, 1H), 5.71-5.65 (m, 1H), $4.72(\mathrm{~s}, 2 \mathrm{H}), 4.58(\mathrm{dd}, J=$ 10.0, $1.2 \mathrm{~Hz}, 2 \mathrm{H}), 4.11(\mathrm{~d}, J=4.8 \mathrm{~Hz}, 2 \mathrm{H}), 3.69(\mathrm{~s}, 3 \mathrm{H}) .{ }^{13} \mathrm{C}$ NMR $(100 \mathrm{MHz}$, D6-DMSO, $\left.80{ }^{\circ} \mathrm{C}\right) \delta 162.2,154.5,137.3,129.9,128.0,126.8,126.6,126.0,123.8$, 121.1, 111.1, 108.2, 66.6, 54.1, 49.6, 47.8. IR (thin film): $v_{\max }\left(\mathrm{cm}^{-1}\right)=3231,3202$, 3030, 2954, 2852, 1743, 1590, 1544, 1256, 940, 790; HRMS (ESI) calcd for $\mathrm{C}_{18} \mathrm{H}_{21} \mathrm{~N}_{2} \mathrm{O}_{4}[\mathrm{M}+\mathrm{H}]^{+}:$329.1496. Found: 329.1487. 
<smiles>COC/C=C/CN(Cc1ccccc1)C(=O)c1cc(Br)c(Br)[nH]1</smiles>

2j. Yellow oil, $43 \%$ yield. ${ }^{1} \mathrm{H}$ NMR (600 MHz, D6-DMSO, $\left.80{ }^{\circ} \mathrm{C}\right) \delta 12.47(\mathrm{~s}, 1 \mathrm{H})$, 7.35-7.33 (m, 2H), 7.28-7.24 (m, 3H), $6.47(\mathrm{~s}, 1 \mathrm{H})$, 5.84-5.80 (m, 1H), 5.71-5.66 (m, $1 \mathrm{H}), 4.67(\mathrm{~s}, 2 \mathrm{H}), 4.57(\mathrm{~d}, J=5.4 \mathrm{~Hz}, 2 \mathrm{H}), 4.07(\mathrm{~d}, J=5.4 \mathrm{~Hz}, 2 \mathrm{H}), 3.71(\mathrm{~s}, 3 \mathrm{H}) .{ }^{13} \mathrm{C}$ NMR (150 MHz, D6-DMSO, $\left.80{ }^{\circ} \mathrm{C}\right) \delta 160.6,154.5,136.9,129.5,128.1,126.9,126.8$, 126.2, 126.1, 113.4, 104.3, 97.4, 66.6, 54.2, 49.5, 47.9. IR (thin film): $v_{\max }\left(\mathrm{cm}^{-1}\right)=$ 3169, 3028, 2958, 2854, 1751, 1591, 1494, 1428, 1255, 1227, 952, 936, 699; HRMS (ESI) calcd for $\mathrm{C}_{18} \mathrm{H}_{19} \mathrm{Br}_{2} \mathrm{~N}_{2} \mathrm{O}_{4}[\mathrm{M}+\mathrm{H}]^{+}$: 484.9706. Found: 484.9692.<smiles>COC/C=C/CN(C(=O)OC)C(=O)c1cc(Br)c(Br)[nH]1</smiles>

2k. White solid, m.p. $=133.4-135.9{ }^{\circ} \mathrm{C}, 53 \%$ yield. ${ }^{1} \mathrm{H}$ NMR $(400 \mathrm{MHz}$, D6-DMSO, $\left.80{ }^{\circ} \mathrm{C}\right) \delta 12.47(\mathrm{~s}, 1 \mathrm{H}), 7.18(\mathrm{~d}, J=8.4 \mathrm{~Hz}, 2 \mathrm{H}), 6.91(\mathrm{~d}, J=8.4 \mathrm{~Hz}, 2 \mathrm{H})$, $6.50(\mathrm{~s}, 1 \mathrm{H}), 5.84-5.79(\mathrm{~m}, 1 \mathrm{H}), 5.72-5.66(\mathrm{~m}, 1 \mathrm{H}), 4.61(\mathrm{~s}, 2 \mathrm{H}), 4.60(\mathrm{~d}, J=10.0 \mathrm{~Hz}$, 2H), $4.06(\mathrm{~d}, J=4.0 \mathrm{~Hz}, 2 \mathrm{H}), 3.76(\mathrm{~s}, 3 \mathrm{H}), 3.72(\mathrm{~s}, 3 \mathrm{H}) .{ }^{13} \mathrm{C}$ NMR (100 MHz, D6-DMSO, $\left.80{ }^{\circ} \mathrm{C}\right) \delta 160.5,158.4,154.5,129.5,128.6,128.2,126.2,126.1,113.7$, 113.3, 104.1, 97.4, 66.5, 54.8, 54.1, 48.9, 47.6. IR (thin film): $v_{\max }\left(\mathrm{cm}^{-1}\right)=3212$, 3009, 2956, 2872, 1743, 1598, 1510, 1441, 1388, 1268, 1245, 978, 904, 699; HRMS (ESI) calcd for $\mathrm{C}_{19} \mathrm{H}_{21} \mathrm{Br}_{2} \mathrm{~N}_{2} \mathrm{O}_{5}[\mathrm{M}+\mathrm{H}]^{+}:$514.9812. Found: 514.9801. 
<smiles>COC/C=C/CN(Cc1ccc(OC)cc1OC)C(=O)c1ccc(Br)[nH]1</smiles>

2l. White solid, m.p. $=109.5-111.0{ }^{\circ} \mathrm{C}, 65 \%$ yield. ${ }^{1} \mathrm{H}$ NMR $(600 \mathrm{MHz}$, D6-DMSO, $\left.80{ }^{\circ} \mathrm{C}\right) \delta 11.96(\mathrm{~s}, 1 \mathrm{H}), 7.03(\mathrm{~d}, J=8.4 \mathrm{~Hz}, 1 \mathrm{H}), 6.56(\mathrm{~d}, J=2.4 \mathrm{~Hz}, 1 \mathrm{H})$, $6.49(\mathrm{dd}, J=8.4,2.4 \mathrm{~Hz}, 1 \mathrm{H}), 6.30(\mathrm{t}, J=3.0 \mathrm{~Hz}, 1 \mathrm{H}), 5.79(\mathrm{dt}, J=15.6,6.0 \mathrm{~Hz}, 1 \mathrm{H})$, $5.64(\mathrm{dt}, J=15.6,6.0 \mathrm{~Hz}, 1 \mathrm{H}), 4.58(\mathrm{~s}, 2 \mathrm{H}), 4.56(\mathrm{dd}, J=6.0,1.2 \mathrm{~Hz}, 2 \mathrm{H}), 4.03(\mathrm{~d}, J$ $=5.4 \mathrm{~Hz}, 2 \mathrm{H}), 3.77(\mathrm{~s}, 3 \mathrm{H}), 3.75(\mathrm{~s}, 3 \mathrm{H}), 3.70(\mathrm{~s}, 3 \mathrm{H}) \cdot{ }^{13} \mathrm{C} \mathrm{NMR}(150 \mathrm{MHz}$, D6-DMSO, $\left.80{ }^{\circ} \mathrm{C}\right) \delta 161.3,159.7,157.8,154.5,130.0,128.3,126.2,125.6,116.9$, $112.3,110.3,104.7,101.7,98.4,66.6,55.1,54.9,54.1,47.4,44.7$. IR (thin film): $v_{\max }$ $\left(\mathrm{cm}^{-1}\right)=3209,3008,2958,2858,1743,1600,1505,1464,1439,1247,1199,979,790$ HRMS (ESI) calcd for $\mathrm{C}_{20} \mathrm{H}_{24} \mathrm{BrN}_{2} \mathrm{O}_{6}[\mathrm{M}+\mathrm{H}]^{+}$: 467.0812. Found: 467.0805.

Scheme S4: General procedure for the synthesis of compound S5:

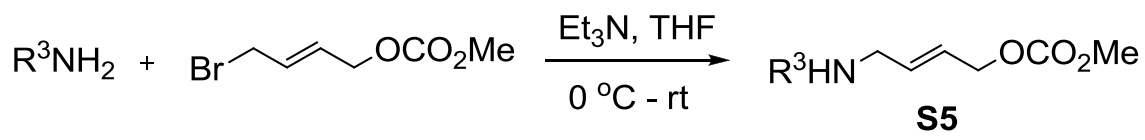

To a solution of the substituted amine ( $48 \mathrm{mmol}, 6.0$ equiv) and $\mathrm{Et}_{3} \mathrm{~N}$ (1.7 mL, $12 \mathrm{mmol}, 1.5$ equiv) in dry THF $(90 \mathrm{~mL})$, carbonic acid $(E)$-4-bromo-but-2-enyl methyl ester $(541 \mathrm{mg}, 2.6 \mathrm{mmol})$ was added dropwise at $0{ }^{\circ} \mathrm{C}$. Then the ice bath was removed and the reaction mixture was stirred at $\mathrm{rt}$ overnight. After the reaction was complete (monitored by TLC), the crude reaction mixture was filtrated with celite and washed with EtOAc. The solvents were removed under reduced pressure. Then the residue was purified by silica gel column chromatography $(\mathrm{PE} / \mathrm{EA}=1 / 1)$ to afford the desired product $\mathbf{S 5}$.<smiles>COC/C=C/CNCc1ccc(OC)cc1</smiles> 
S5-1. Yellow oil, $76 \%$ yield. ${ }^{1} \mathrm{H}$ NMR $\left(300 \mathrm{MHz}, \mathrm{CDCl}_{3}\right) \delta 7.22(\mathrm{~d}, J=8.7 \mathrm{~Hz}$, 2H), $6.85(\mathrm{~d}, J=8.1 \mathrm{~Hz}, 2 \mathrm{H}), 5.95-5.86(\mathrm{~m}, 1 \mathrm{H}), 5.80-5.71(\mathrm{~m}, 1 \mathrm{H}), 4.61(\mathrm{~d}, J=6.0$ $\mathrm{Hz}, 2 \mathrm{H}), 3.78$ (s, 3H), $3.76(\mathrm{~s}, 3 \mathrm{H}), 3.70(\mathrm{~s}, 2 \mathrm{H}), 3.26$ (d, J = 5.7 Hz, 2H), 1.51 (br s, 1H). ${ }^{13} \mathrm{C} \mathrm{NMR}\left(75 \mathrm{MHz}, \mathrm{CDCl}_{3}\right) \delta 158.5,155.4,134.2$, 132.0, 129.2, 124.6, 113.6, 67.8, 55.0, 54.6, 52.5, 49.9. IR (thin film): $v_{\max }\left(\mathrm{cm}^{-1}\right)=2962,2926,2853,1749,1612$, 1513, 1444, 1259, 1089, 1016, 795; HRMS (ESI) calcd for $\mathrm{C}_{14} \mathrm{H}_{20} \mathrm{NO}_{4}[\mathrm{M}+\mathrm{H}]^{+}$: 266.1387. Found: 266.1389.<smiles>COc1ccc(CNC/C=C/COC(C)=O)c(OC)c1</smiles>

S5-2. Yellow oil, $73 \%$ yield. ${ }^{1} \mathrm{H}$ NMR $\left(400 \mathrm{MHz}, \mathrm{CDCl}_{3}\right) \delta 6.97(\mathrm{~d}, J=8.0 \mathrm{~Hz}$, $1 \mathrm{H}), 6.32-6.27(\mathrm{~m}, 2 \mathrm{H}), 5.80-5.73(\mathrm{~m}, 1 \mathrm{H}), 5.65-5.58(\mathrm{~m}, 1 \mathrm{H}), 4.46(\mathrm{~d}, J=6.0 \mathrm{~Hz}$, 2H), 3.65 (s, 3H), 3.63 (s, 3H), 3.63 (br s, 1H), 3.61 (s, 3H), 3.57 (s, 2H), 3.09 (d, J = $6.0 \mathrm{~Hz}, 2 \mathrm{H}) .{ }^{13} \mathrm{C} \mathrm{NMR}\left(100 \mathrm{MHz}, \mathrm{CDCl}_{3}\right) \delta 159.6,158.0,155.0,134.2,129.9,124.0$, 120.0, 103.0, 97.9, 67.5, 54.63, 54.60, 54.1, 49.4, 47.7. IR (thin film): $v_{\max }\left(\mathrm{cm}^{-1}\right)=$ $3612,3003,2955,2836,1745,1612,1588,1506,1440,1419,1256,1207,937,791$; HRMS (ESI) calcd for $\mathrm{C}_{15} \mathrm{H}_{22} \mathrm{NO}_{5}[\mathrm{M}+\mathrm{H}]^{+}:$296.1492. Found: 296.1487.

Scheme S5: General procedure for iridium-catalyzed intramolecular enantioselective allylic amination of pyrroles:<smiles>[R]c1cc(C([X])[X])[nH]c1[R]</smiles>
$[\mathrm{Ir}(\mathrm{cod}) \mathrm{Cl}]_{2}(2 \mathrm{~mol} \%)$ $\underset{\mathrm{DBU}(100 \mathrm{~mol} \%)}{\stackrel{\left(S, S, S_{a}\right)-1 \mathbf{b}(4 \mathrm{~mol} \%)}{\longrightarrow}}$ $\mathrm{THF}, 50^{\circ} \mathrm{C}$

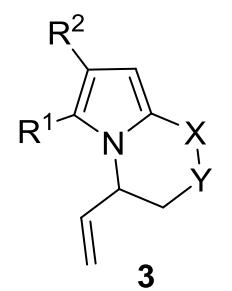

3

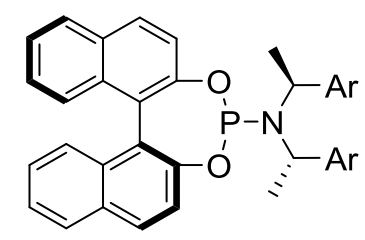

$\left(S, S, S_{a}\right)-1 \mathbf{b}: \mathrm{Ar}=2-\mathrm{MeO}-\mathrm{C}_{6} \mathrm{H}_{4}$

A flame-dried Schlenk tube was cooled to room temperature and filled with argon. To this flask were added $[\operatorname{Ir}(\operatorname{cod}) \mathrm{Cl}]_{2}(2.7 \mathrm{mg}, 0.004 \mathrm{mmol}, 2 \mathrm{~mol} \%)$, Alexakis 
ligand $\mathbf{1 b}(4.8 \mathrm{mg}, 0.008 \mathrm{mmol}, 4 \mathrm{~mol} \%)$, THF (0.5 mL) and $n$-propylamine $(0.3 \mathrm{~mL})$. The reaction mixture was heated at $50{ }^{\circ} \mathrm{C}$ for $30 \mathrm{~min}$ and then the volatile solvents were removed in vacuo to give a yellow solid. After that, allylic carbonate $2(0.20$ mmol, dissolved in $2.0 \mathrm{~mL}$ THF) and DBU (30 $\mu \mathrm{L}, 0.20 \mathrm{mmol}, 100 \mathrm{~mol} \%)$ were added. The reaction mixture was heated at $50{ }^{\circ} \mathrm{C}$. After the reaction was complete (monitored by TLC), the crude reaction mixture was filtrated with celite and washed with DCM. The solvents were removed under reduced pressure. Then the residue was purified by silica gel column chromatography $(\mathrm{PE} / \mathrm{EtOAc}=6 / 1)$ to afford the desired product $\mathbf{3}$. The characterization of the products $\mathbf{3}$ are summarized below.

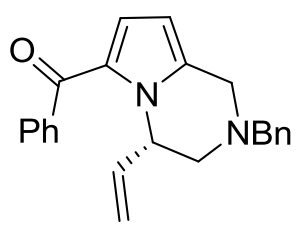

3a. Yellow solid, m.p. $=112.3-114.8{ }^{\circ} \mathrm{C}, 55.7 \mathrm{mg}, 81 \%$ yield, $98 \%$ ee [Phenomenex Lux 5u Celluloxe-2 PC-2 $(0.46 \mathrm{~cm}$ x $25 \mathrm{~cm}), n$-hexane $/ 2-$ propanol $=$ $70 / 30, v=0.5 \mathrm{~mL} \cdot \min ^{-1}, \lambda=254 \mathrm{~nm}, \mathrm{t}($ minor $)=11.03 \mathrm{~min}, \mathrm{t}$ (major) $\left.=9.71 \mathrm{~min}\right]$; $[\alpha]_{\mathrm{D}}{ }^{20}=+153.3\left(\mathrm{c}=1.0, \mathrm{CHCl}_{3}\right) .{ }^{1} \mathrm{H} \mathrm{NMR}\left(300 \mathrm{MHz}, \mathrm{CDCl}_{3}\right) \delta 7.76(\mathrm{~d}, J=7.8 \mathrm{~Hz}$, 2H), 7.52-7.24 (m, 8H), $6.71(\mathrm{~d}, J=4.2 \mathrm{~Hz}, 1 \mathrm{H}), 6.07(\mathrm{ddd}, J=16.8,10.5,6.3 \mathrm{~Hz}$, 1H), $5.90(\mathrm{~d}, J=4.2 \mathrm{~Hz}, 1 \mathrm{H}), 5.78(\mathrm{~m}, 1 \mathrm{H}), 5.05(\mathrm{~d}, J=10.2 \mathrm{~Hz}, 1 \mathrm{H}), 4.98(\mathrm{~d}, J=$ $17.1 \mathrm{~Hz}, 1 \mathrm{H}), 4.04(\mathrm{~d}, J=15.0 \mathrm{~Hz}, 1 \mathrm{H}), 3.72\left(\mathrm{AB}, J_{A B}=13.2 \mathrm{~Hz}, 1 \mathrm{H}\right), 3.63\left(\mathrm{BA}, J_{B A}\right.$ $=13.5 \mathrm{~Hz}, 1 \mathrm{H}), 3.42(\mathrm{~d}, J=15.0 \mathrm{~Hz}, 1 \mathrm{H}), 3.02(\mathrm{~d}, J=11.7 \mathrm{~Hz}, 1 \mathrm{H}), 2.82(\mathrm{dd}, J=$ 12.0, $3.9 \mathrm{~Hz}, 1 \mathrm{H}) .{ }^{13} \mathrm{C} \mathrm{NMR}\left(75 \mathrm{MHz}, \mathrm{CDCl}_{3}\right) \delta 185.1,140.1,138.4,137.5,136.7$, 131.1, 129.1, 128.9, 128.8, 128.4, 127.9, 127.4, 124.0, 115.5, 105.0, 62.2, 56.7, 55.0, 51.3. IR (thin film): $v_{\max }\left(\mathrm{cm}^{-1}\right)=3008,2959,2926,1630,1574,1537,1482,1261$, 928, 697; HRMS (ESI) calcd for $\mathrm{C}_{23} \mathrm{H}_{23} \mathrm{~N}_{2} \mathrm{O}[\mathrm{M}+\mathrm{H}]^{+}:$343.1805. Found: 343.1795.

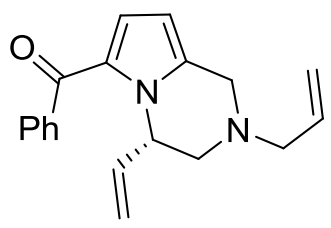


3b. Pale yellow oil, $30.9 \mathrm{mg}, 86 \%$ yield, 98\% ee [Phenomenex Lux 5u Celluloxe-2 PC-2 $(0.46 \mathrm{~cm} \mathrm{x} 25 \mathrm{~cm}), n$-hexane/2-propanol $=70 / 30, v=0.5 \mathrm{~mL} \cdot \mathrm{min}^{-1}$, $\lambda=230 \mathrm{~nm}, \mathrm{t}$ (minor) $=9.57 \mathrm{~min}, \mathrm{t}$ (major) $=8.73 \mathrm{~min}] ;[\alpha]_{\mathrm{D}}{ }^{27}=+183.1(\mathrm{c}=1.0$, $\left.\mathrm{CHCl}_{3}\right) .{ }^{1} \mathrm{H} \mathrm{NMR}\left(400 \mathrm{MHz}, \mathrm{CDCl}_{3}\right) \delta 7.50(\mathrm{~d}, J=7.2 \mathrm{~Hz}, 2 \mathrm{H}), 7.50-7.48(\mathrm{~m}, 1 \mathrm{H})$, 7.44-7.40 (m, 2H), $6.72(\mathrm{~d}, J=4.0 \mathrm{~Hz}, 1 \mathrm{H}), 6.05$ (ddd, $J=16.4,10.0,6.0 \mathrm{~Hz}, 1 \mathrm{H})$, $5.94(\mathrm{~d}, J=4.0 \mathrm{~Hz}, 1 \mathrm{H}), 5.89-5.83(\mathrm{~m}, 1 \mathrm{H}), 5.79(\mathrm{~m}, 1 \mathrm{H}), 5.28(\mathrm{~d}, J=17.6 \mathrm{~Hz}, 1 \mathrm{H})$, $5.22(\mathrm{~d}, J=10.0 \mathrm{~Hz}, 1 \mathrm{H}), 5.06(\mathrm{~d}, J=10.4 \mathrm{~Hz}, 1 \mathrm{H}), 4.97(\mathrm{~d}, J=17.2 \mathrm{~Hz}, 1 \mathrm{H}), 4.06(\mathrm{~d}$, $J=15.2 \mathrm{~Hz}, 1 \mathrm{H}), 3.39(\mathrm{~d}, J=15.2 \mathrm{~Hz}, 1 \mathrm{H}), 3.21(\mathrm{dd}, J=13.2 \mathrm{~Hz}, 5.6 \mathrm{~Hz}, 1 \mathrm{H})$, 3.11-3.08 (m, 2H), $2.75(\mathrm{dd}, J=12.0,4.0 \mathrm{~Hz}, 1 \mathrm{H}) .{ }^{13} \mathrm{C} \mathrm{NMR}\left(100 \mathrm{MHz}, \mathrm{CDCl}_{3}\right) \delta$ $185.1,140.2$, 138.6, 136.8, 134.4, 131.1, 129.2, 129.1, 127.9, 123.9, 118.4, 115.4, 105.0, 60.9, 56.7, 54.9, 51.4. IR (thin film): $v_{\max }\left(\mathrm{cm}^{-1}\right)=3064,2959,2930,1730$, 1630, 1574, 1537, 1446, 1262, 1157, 811, 699; HRMS (ESI) calcd for $\mathrm{C}_{19} \mathrm{H}_{21} \mathrm{~N}_{2} \mathrm{O}$ $[\mathrm{M}+\mathrm{H}]^{+}:$293.1648. Found: 293.1647 .

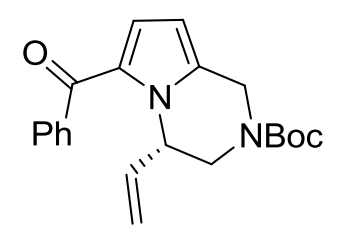

3c. Pale yellow oil, $54.2 \mathrm{mg}, 77 \%$ yield, $94 \%$ ee [Daicel Chiralpak AD-H (0.46 $\mathrm{cm} \times 25 \mathrm{~cm}$ ), $n$-hexane/2-propanol $=90 / 10, v=0.5 \mathrm{~mL} \cdot \min ^{-1}, \lambda=230 \mathrm{~nm}, \mathrm{t}$ (minor) $=17.89 \mathrm{~min}, \mathrm{t}$ (major) $=13.59 \mathrm{~min}] ;[\alpha]_{\mathrm{D}}{ }^{26}=+75.0\left(\mathrm{c}=1.0, \mathrm{CHCl}_{3}\right) .{ }^{1} \mathrm{H} \mathrm{NMR}(400$ MHz, D6-DMSO, $\left.80{ }^{\circ} \mathrm{C}\right) \delta 7.66(\mathrm{~d}, J=7.6 \mathrm{~Hz}, 2 \mathrm{H}), 7.56(\mathrm{t}, J=7.6 \mathrm{~Hz}, 1 \mathrm{H}), 7.48(\mathrm{t}, J$ $=7.6 \mathrm{~Hz}, 2 \mathrm{H}), 6.70(\mathrm{~d}, J=4.0 \mathrm{~Hz}, 1 \mathrm{H}), 6.13(\mathrm{~d}, J=3.6 \mathrm{~Hz}, 1 \mathrm{H}), 5.98(\mathrm{ddd}, J=15.6$, 10.4, $4.8 \mathrm{~Hz}, 1 \mathrm{H}), 5.87$ (m, 1H), 5.05 (d, $J=10.4 \mathrm{~Hz}, 1 \mathrm{H}), 4.93$ (d, $J=17.6 \mathrm{~Hz}, 1 \mathrm{H})$, $4.60(\mathrm{~d}, J=16.8 \mathrm{~Hz}, 1 \mathrm{H}), 4.41(\mathrm{~d}, J=17.6 \mathrm{~Hz}, 1 \mathrm{H}), 4.27$ (d, $J=13.6 \mathrm{~Hz}, 1 \mathrm{H}), 3.46$ (d, $J=13.6 \mathrm{~Hz}, 1 \mathrm{H}), 1.44(\mathrm{~s}, 9 \mathrm{H}) .{ }^{13} \mathrm{C} \mathrm{NMR}\left(100 \mathrm{MHz}, \mathrm{D} 6-\mathrm{DMSO}, 80{ }^{\circ} \mathrm{C}\right) \delta 183.7$, 153.6, 139.5, 136.9, 134.1, 130.7, 128.2, 128.0, 127.7, 122.5, 114.4, 105.0, 79.2, 55.0, 44.3, 40.8, 27.6. IR (thin film): $v_{\max }\left(\mathrm{cm}^{-1}\right)=3005,2973,2927,1700,1628,1576$, 1477, 1237, 883, 724; HRMS (ESI) calcd for $\mathrm{C}_{21} \mathrm{H}_{25} \mathrm{~N}_{2} \mathrm{O}_{3}[\mathrm{M}+\mathrm{H}]^{+}: 353.1860$. Found: 353.1849 . 


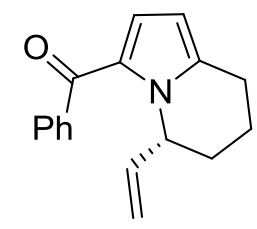

3d. White solid, m.p. $=73.5-75.7^{\circ} \mathrm{C}, 38.1 \mathrm{mg}, 72 \%$ yield, $95 \%$ ee $[$ Phenomenex Lux 5u Amylose-2 PA-2 (0.46 cm x $25 \mathrm{~cm}), n$-hexane/2-propanol $=90 / 10, v=0.5$ $\mathrm{mL} \cdot \min ^{-1}, \lambda=230 \mathrm{~nm}, \mathrm{t}($ minor $)=26.18 \mathrm{~min}, \mathrm{t}($ major $\left.)=21.88 \mathrm{~min}\right] ;[\alpha]_{\mathrm{D}}^{25}=+238.8$ $\left(\mathrm{c}=1.0, \mathrm{CHCl}_{3}\right) .{ }^{1} \mathrm{H} \mathrm{NMR}\left(300 \mathrm{MHz}, \mathrm{CDCl}_{3}\right) \delta 7.64(\mathrm{~d}, J=6.9 \mathrm{~Hz}, 2 \mathrm{H}), 7.42-7.31$ $(\mathrm{m}, 3 \mathrm{H}), 6.64(\mathrm{~d}, J=3.9 \mathrm{~Hz}, 1 \mathrm{H}), 5.95-5.83(\mathrm{~m}, 3 \mathrm{H}), 4.98(\mathrm{~d}, J=9.9 \mathrm{~Hz}, 1 \mathrm{H}), 4.48(\mathrm{~d}$, $J=16.5 \mathrm{~Hz}, 1 \mathrm{H}), 2.89(\mathrm{dd}, J=17.4,5.7 \mathrm{~Hz}, 1 \mathrm{H}), 2.77-2.64(\mathrm{~m}, 1 \mathrm{H}), 2.10-2.00(\mathrm{~m}$, 1H), $1.93-1.65(\mathrm{~m}, 3 \mathrm{H}), 1.21-1.18(\mathrm{~m}, 1 \mathrm{H}) .{ }^{13} \mathrm{C} \mathrm{NMR}\left(75 \mathrm{MHz}, \mathrm{CDCl}_{3}\right) \delta 184.9$, 140.6, 139.7, 139.6, 130.8, 129.1, 127.8, 124.2, 114.2, 106.7, 55.4, 27.6, 23.8, 15.1. IR (thin film): $v_{\max }\left(\mathrm{cm}^{-1}\right)=3082,2927,2854,1722,1623,1517,1444,1236,880$, 724, 698; HRMS (ESI) calcd for $\mathrm{C}_{17} \mathrm{H}_{18} \mathrm{NO}[\mathrm{M}+\mathrm{H}]^{+}:$252.1383. Found: 252.1381.

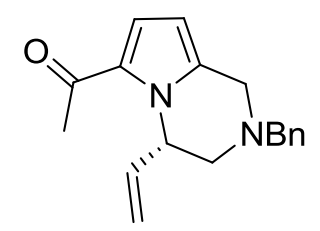

3e. Pale yellow oil, $16.2 \mathrm{mg}, 58 \%$ yield, $97 \%$ ee [Daicel Chiralpak IC $(0.46 \mathrm{~cm} \mathrm{x}$ $25 \mathrm{~cm}), n$-hexane $/ 2$-propanol $=98 / 2, v=0.5 \mathrm{~mL} \cdot \min ^{-1}, \lambda=230 \mathrm{~nm}, \mathrm{t}($ minor $)=29.64$ $\min , \mathrm{t}($ major $)=39.03 \mathrm{~min}] ;[\alpha]_{\mathrm{D}}{ }^{27}=+151.2\left(\mathrm{c}=1.0, \mathrm{CHCl}_{3}\right) .{ }^{1} \mathrm{H} \mathrm{NMR}(400 \mathrm{MHz}$, $\left.\mathrm{CDCl}_{3}\right) \delta 7.34-7.26(\mathrm{~m}, 5 \mathrm{H}), 6.95(\mathrm{~d}, J=4.0 \mathrm{~Hz}, 1 \mathrm{H}), 6.00(\mathrm{ddd}, J=16.4,10.4,6.0$ $\mathrm{Hz}, 1 \mathrm{H}), 5.88(\mathrm{~d}, J=4.4 \mathrm{~Hz}, 1 \mathrm{H}), 5.59(\mathrm{t}, J=3.6 \mathrm{~Hz}, 1 \mathrm{H}), 5.06(\mathrm{dd}, J=10.4,3.6 \mathrm{~Hz}$, $1 \mathrm{H}), 4.87-4.82(\mathrm{~m}, 1 \mathrm{H}), 4.03(\mathrm{dd}, J=15.2,1.2 \mathrm{~Hz}, 1 \mathrm{H}), 3.70\left(\mathrm{AB}, J_{A B}=13.6 \mathrm{~Hz}, 1 \mathrm{H}\right)$, $3.60\left(\mathrm{BA}, J_{B A}=13.6 \mathrm{~Hz}, 1 \mathrm{H}\right), 3.37(\mathrm{~d}, J=14.8 \mathrm{~Hz}, 1 \mathrm{H}), 3.00(\mathrm{dt}, J=12.0,1.6 \mathrm{~Hz}$, $1 \mathrm{H}), 2.71(\mathrm{dd}, J=12.4,4.0 \mathrm{~Hz}, 1 \mathrm{H}), 2.36(\mathrm{~s}, 3 \mathrm{H}) .{ }^{13} \mathrm{C} \mathrm{NMR}\left(100 \mathrm{MHz}, \mathrm{CDCl}_{3}\right) \delta$ 187.0, 138.4, 137.5, 135.9, 129.3, 128.8, 128.4, 127.3, 120.5, 115.1, 104.7, 62.3, 56.8, 54.8, 51.3, 27.0. IR (thin film): $v_{\max }\left(\mathrm{cm}^{-1}\right)=2963,2852,1644,1537,1262,1101$, 1027, 799, 699; HRMS (ESI) calcd for $\mathrm{C}_{18} \mathrm{H}_{21} \mathrm{~N}_{2} \mathrm{O}[\mathrm{M}+\mathrm{H}]^{+}$: 281.1648. Found: 


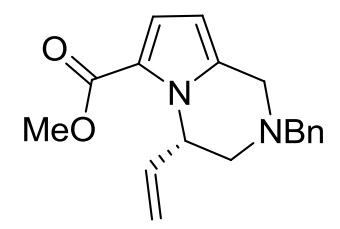

3f. Pale yellow oil, $25.6 \mathrm{mg}, 86 \%$ yield, $98 \%$ ee [Phenomenex Lux 5u Celluloxe-2 PC-2 $(0.46 \mathrm{~cm} \times 25 \mathrm{~cm}), n$-hexane $/ 2$-propanol $=70 / 30, v=0.5 \mathrm{~mL} \cdot \mathrm{min}^{-1}$, $\lambda=230 \mathrm{~nm}, \mathrm{t}$ (minor) $=7.45 \mathrm{~min}, \mathrm{t}$ (major) $=7.86 \mathrm{~min}] ;[\alpha]_{\mathrm{D}}{ }^{25}=+37.3(\mathrm{c}=1.0$, $\left.\mathrm{CHCl}_{3}\right) .{ }^{1} \mathrm{H} \mathrm{NMR}\left(400 \mathrm{MHz}, \mathrm{CDCl}_{3}\right) \delta$ 7.35-7.25 (m, 5H), $6.94(\mathrm{~d}, J=3.6 \mathrm{~Hz}, 1 \mathrm{H})$, 6.01 (ddd, $J=17.2,10.4,6.0 \mathrm{~Hz}, 1 \mathrm{H}), 5.86$ (d, $J=3.6 \mathrm{~Hz}, 1 \mathrm{H}), 5.50-5.43(\mathrm{~m}, 1 \mathrm{H})$, $5.08(\mathrm{~d}, J=10.4 \mathrm{~Hz}, 1 \mathrm{H}), 4.84(\mathrm{~d}, J=17.2 \mathrm{~Hz}, 1 \mathrm{H}), 4.02(\mathrm{~d}, J=14.8 \mathrm{~Hz}, 1 \mathrm{H}), 3.75$ (s, $3 \mathrm{H}), 3.70\left(\mathrm{AB}, J_{A B}=13.2 \mathrm{~Hz}, 1 \mathrm{H}\right), 3.59\left(\mathrm{BA}, J_{B A}=13.2 \mathrm{~Hz}, 1 \mathrm{H}\right), 3.36(\mathrm{~d}, J=14.8 \mathrm{~Hz}$, 1H), $3.01(\mathrm{~d}, J=11.6 \mathrm{~Hz}, 1 \mathrm{H}), 2.72(\mathrm{dd}, J=12.0,3.6 \mathrm{~Hz}, 1 \mathrm{H}) .{ }^{13} \mathrm{C} \mathrm{NMR}(100 \mathrm{MHz}$, $\left.\mathrm{CDCl}_{3}\right) \delta 161.0,138.5,137.5,134.5,128.8,128.3,127.3,120.4,118.3,115.2,104.4$, 62.2, 56.5, 54.9, 51.3, 50.8. IR (thin film): $v_{\max }\left(\mathrm{cm}^{-1}\right)=3027,3007,2955,2853,1707$, 1649, 1234, 748, 699; HRMS (ESI) calcd for $\mathrm{C}_{18} \mathrm{H}_{21} \mathrm{~N}_{2} \mathrm{O}_{2}[\mathrm{M}+\mathrm{H}]^{+}:$297.1598. Found: 297.1593.

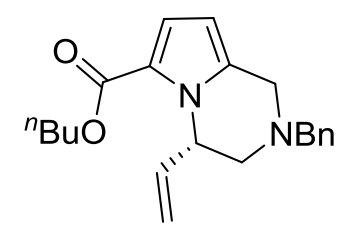

3g. Pale yellow oil, $55.4 \mathrm{mg}, 82 \%$ yield, $98 \%$ ee [Daicel Chiralcel OD-H (0.46 $\mathrm{cm} \times 25 \mathrm{~cm}$ ), $n$-hexane/2-propanol $=95 / 5, v=0.6 \mathrm{~mL} \cdot \min ^{-1}, \lambda=230 \mathrm{~nm}, \mathrm{t}$ (minor) $=$ $9.88 \mathrm{~min}, \mathrm{t}($ major $)=8.33 \mathrm{~min}] ;[\alpha]_{\mathrm{D}}{ }^{25}=+44.0\left(\mathrm{c}=1.0, \mathrm{CHCl}_{3}\right) .{ }^{1} \mathrm{H} \mathrm{NMR}(400 \mathrm{MHz}$, $\left.\mathrm{CDCl}_{3}\right) \delta 7.35-7.24(\mathrm{~m}, 5 \mathrm{H}), 6.94(\mathrm{~d}, J=3.6 \mathrm{~Hz}, 1 \mathrm{H}), 6.00(\mathrm{ddd}, J=16.8,10.4,6.0$ $\mathrm{Hz}, 1 \mathrm{H}), 5.85$ (d, $J=4.0 \mathrm{~Hz}, 1 \mathrm{H}), 5.47$ (br s, 1H), 5.08 (d, $J=10.4 \mathrm{~Hz}, 1 \mathrm{H}), 4.83$ (d, $J$ $=17.2 \mathrm{~Hz}, 1 \mathrm{H}), 4.23-4.03(\mathrm{~m}, 2 \mathrm{H}), 4.02(\mathrm{~d}, J=14.8 \mathrm{~Hz}, 1 \mathrm{H}), 3.70\left(\mathrm{AB}, J_{A B}=13.2 \mathrm{~Hz}\right.$, 1H), $3.58\left(\mathrm{BA}, J_{B A}=13.2 \mathrm{~Hz}, 1 \mathrm{H}\right), 3.35(\mathrm{~d}, J=14.4 \mathrm{~Hz}, 1 \mathrm{H}), 3.00(\mathrm{~d}, J=11.6 \mathrm{~Hz}$, 1H), $2.71(\mathrm{dd}, J=12.0,3.6 \mathrm{~Hz}, 1 \mathrm{H}), 1.70-1.63(\mathrm{~m}, 2 \mathrm{H}), 1.44-1.39(\mathrm{~m}, 2 \mathrm{H}), 0.94(\mathrm{t}, J$ 
$=7.2 \mathrm{~Hz}, 1 \mathrm{H}) .{ }^{13} \mathrm{C} \mathrm{NMR}\left(100 \mathrm{MHz}, \mathrm{CDCl}_{3}\right) \delta 160.7,138.5,137.5,134.3,128.7$, 128.3, 127.3, 120.8, 118.1, 115.1, 104.3, 63.3, 62.2, 56.4, 54.9, 51.4, 30.8, 19.2, 13.7. IR (thin film): $v_{\max }\left(\mathrm{cm}^{-1}\right)=2960,2932,1705,1653,1541,1236,801,749$; HRMS (ESI) calcd for $\mathrm{C}_{21} \mathrm{H}_{27} \mathrm{~N}_{2} \mathrm{O}_{2}[\mathrm{M}+\mathrm{H}]^{+}$: 339.2067. Found: 339.2065.

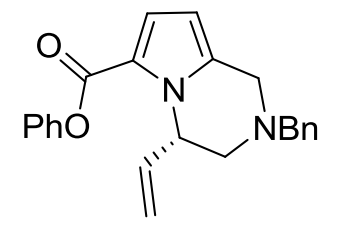

3h. Pale yellow oil, $30.7 \mathrm{mg}, 87 \%$ yield, 97\% ee [Phenomenex Lux 5u Celluloxe-2 PC-2 $(0.46 \mathrm{~cm} \mathrm{x} 25 \mathrm{~cm}), n$-hexane $/ 2$-propanol $=70 / 30, v=0.5 \mathrm{~mL} \cdot \mathrm{min}^{-1}$, $\lambda=230 \mathrm{~nm}, \mathrm{t}$ (minor) $=7.83 \mathrm{~min}, \mathrm{t}$ (major) $=8.35 \mathrm{~min}] ;[\alpha]_{\mathrm{D}}{ }^{25}=+53.6(\mathrm{c}=1.0$, $\left.\mathrm{CHCl}_{3}\right) .{ }^{1} \mathrm{H}$ NMR (400 MHz, $\left.\mathrm{CDCl}_{3}\right) \delta$ 7.39-7.27 (m, 7H), 7.23-7.12 (m, 4H), 6.00 (ddd, $J=16.4,10.0,5.6 \mathrm{~Hz}, 1 \mathrm{H}), 5.95$ (d, $J=3.6 \mathrm{~Hz}, 1 \mathrm{H}), 5.49-5.41(\mathrm{~m}, 1 \mathrm{H}), 5.10$ (d, $J=10.0 \mathrm{~Hz}, 1 \mathrm{H}), 4.92(\mathrm{~d}, J=17.2 \mathrm{~Hz}, 1 \mathrm{H}), 4.05(\mathrm{~d}, J=14.8 \mathrm{~Hz}, 1 \mathrm{H}), 3.72\left(\mathrm{AB}, J_{A B}\right.$ $=13.2 \mathrm{~Hz}, 1 \mathrm{H}), 3.60\left(\mathrm{BA}, J_{B A}=13.2 \mathrm{~Hz}, 1 \mathrm{H}\right), 3.39(\mathrm{~d}, J=14.8 \mathrm{~Hz}, 1 \mathrm{H}), 3.01(\mathrm{~d}, J=$ $12.0 \mathrm{~Hz}, 1 \mathrm{H}), 2.74(\mathrm{dd}, J=11.6,3.6 \mathrm{~Hz}, 1 \mathrm{H}) .{ }^{13} \mathrm{C} \mathrm{NMR}\left(100 \mathrm{MHz}, \mathrm{CDCl}_{3}\right) \delta 158.8$, 150.7, 138.2, 137.5, 135.8, 129.3, 128.8, 128.4, 127.3, 125.3, 121.9, 119.8, 119.7, 115.6, 105.0, 62.2, 56.6, 54.9, 51.3. IR (thin film): $v_{\max }\left(\mathrm{cm}^{-1}\right)=2962,2919,2850$, $1715,1653,1481,1262,1194,800,737$; HRMS (ESI) calcd for $\mathrm{C}_{23} \mathrm{H}_{23} \mathrm{~N}_{2} \mathrm{O}_{2}[\mathrm{M}+\mathrm{H}]^{+}$: 359.1754. Found: 359.1751.

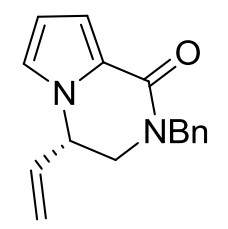

3i. Yellow oil, $19.6 \mathrm{mg}, 78 \%$ yield, $96 \%$ ee [Daicel Chiralpak AD-H (0.46 cm x $25 \mathrm{~cm}$ ), $n$-hexane/2-propanol $=90 / 10, v=0.5 \mathrm{~mL} \cdot \min ^{-1}, \lambda=230 \mathrm{~nm}, \mathrm{t}$ (minor) $=$ $23.97 \mathrm{~min}, \mathrm{t}($ major $)=26.84 \mathrm{~min}] ;[\alpha]_{\mathrm{D}}{ }^{20}=-1.7\left(\mathrm{c}=1.0, \mathrm{CHCl}_{3}\right) .{ }^{1} \mathrm{H} \mathrm{NMR}(400 \mathrm{MHz}$, $\left.\mathrm{CDCl}_{3}\right) \delta$ 7.33-7.26 (m, 5H), 7.03-7.02 (m, 1H), 6.72-6.71 (m, 1H), 6.26-6.25 (m, 1H), 
5.77 (ddd, $J=16.4,9.6,6.4 \mathrm{~Hz}, 1 \mathrm{H}), 5.34(\mathrm{~d}, J=10.0 \mathrm{~Hz}, 1 \mathrm{H}), 5.12(\mathrm{~d}, J=17.2 \mathrm{~Hz}$, $1 \mathrm{H}), 4.76\left(\mathrm{AB}, J_{A B}=14.8 \mathrm{~Hz}, 1 \mathrm{H}\right), 4.69\left(\mathrm{BA}, J_{B A}=14.8 \mathrm{~Hz}, 1 \mathrm{H}\right), 4.68-4.61(\mathrm{~m}, 1 \mathrm{H})$, $3.55(\mathrm{dd}, J=12.8,4.8 \mathrm{~Hz}, 1 \mathrm{H}), 3.42(\mathrm{dd}, J=12.8,7.6 \mathrm{~Hz}, 1 \mathrm{H}) .{ }^{13} \mathrm{C} \mathrm{NMR}(100 \mathrm{MHz}$, $\left.\mathrm{CDCl}_{3}\right) \delta 159.4,136.9,133.6,128.6,128.3,127.6,124.2,122.0,120.8,114.1,110.0$, 56.7, 50.0, 49.1. IR (thin film): $v_{\max }\left(\mathrm{cm}^{-1}\right)=3008,2960,2855,1634,1544,1474$, 1076, 732, 699; HRMS (ESI) calcd for $\mathrm{C}_{16} \mathrm{H}_{17} \mathrm{~N}_{2} \mathrm{O}[\mathrm{M}+\mathrm{H}]^{+}:$253.1335. Found: 253.1328 .<smiles>C=C[C@H]1C[NH2+]C(Cc2ccccc2)C(=O)c2cc(Br)c(Br)n21</smiles>

3j. White solid, m.p. $=69.5-71.9{ }^{\circ} \mathrm{C}, 33.5 \mathrm{mg}, 82 \%$ yield, 93\% ee [Daicel Chiralpak AD-H $(0.46 \mathrm{~cm} \times 25 \mathrm{~cm}), n$-hexane/2-propanol $=90 / 10, v=0.6 \mathrm{~mL} \cdot \mathrm{min}^{-1}$, $\lambda=230 \mathrm{~nm}, \mathrm{t}$ (minor) $=37.31 \mathrm{~min}, \mathrm{t}($ major $)=29.82 \mathrm{~min}] ;[\alpha]_{\mathrm{D}}^{23}=-25.3(\mathrm{c}=1.0$, $\left.\mathrm{CHCl}_{3}\right) .{ }^{1} \mathrm{H} \mathrm{NMR}\left(400 \mathrm{MHz}, \mathrm{CDCl}_{3}\right) \delta$ 7.34-7.25 (m, 5H), $7.06(\mathrm{~s}, 1 \mathrm{H}), 5.60$ (ddd, $J=$ 15.6, 10.8, $5.2 \mathrm{~Hz}, 1 \mathrm{H}), 5.17$ (d, $J=10.4 \mathrm{~Hz}, 1 \mathrm{H}), 4.84(\mathrm{~d}, J=14.4 \mathrm{~Hz}, 1 \mathrm{H})$, 4.76-4.74 (m, 1H), 4.65 (dd, $J=16.8,1.2 \mathrm{~Hz}, 1 \mathrm{H}), 4.52$ (d, $J=14.8 \mathrm{~Hz}, 1 \mathrm{H}), 3.88$ (dd, $J=13.2,4.4 \mathrm{~Hz}, 1 \mathrm{H}), 3.33(\mathrm{dd}, J=13.2,0.8 \mathrm{~Hz}, 1 \mathrm{H}) .{ }^{13} \mathrm{C} \mathrm{NMR}\left(100 \mathrm{MHz}, \mathrm{CDCl}_{3}\right) \delta$ 157.4, 136.0, 133.0, 128.6, 128.5, 127.8, 125.5, 118.6, 115.8, 106.6, 100.8, 55.4, 49.4, 48.9. IR (thin film): $v_{\max }\left(\mathrm{cm}^{-1}\right)=3086,2921,2854,1640,1542,1422,1137,743,698$; HRMS (ESI) calcd for $\mathrm{C}_{16} \mathrm{H}_{15} \mathrm{Br}_{2} \mathrm{~N}_{2} \mathrm{O}[\mathrm{M}+\mathrm{H}]^{+}$: 408.9546. Found: 408.9541.

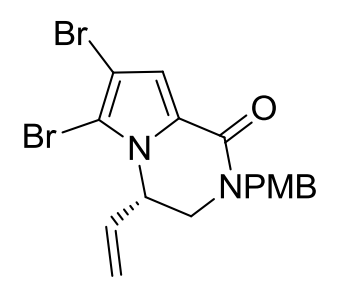

3k. Yellow oil, $83.3 \mathrm{mg}, 96 \%$ yield, $96 \%$ ee [Daicel Chiralpak AD-H (0.46 cm x $25 \mathrm{~cm}$ ), $n$-hexane/2-propanol $=80 / 20, v=0.8 \mathrm{~mL} \cdot \min ^{-1}, \lambda=254 \mathrm{~nm}, \mathrm{t}$ (minor) $=$ $20.97 \mathrm{~min}, \mathrm{t}$ (major) $=15.52 \mathrm{~min}] ;[\alpha]_{\mathrm{D}}{ }^{25}=-26.6\left(\mathrm{c}=1.0, \mathrm{CHCl}_{3}\right) .{ }^{1} \mathrm{H} \mathrm{NMR}(400$ $\left.\mathrm{MHz}, \mathrm{CDCl}_{3}\right) \delta 7.19(\mathrm{~d}, J=8.4 \mathrm{~Hz}, 1 \mathrm{H}), 7.04(\mathrm{~s}, 1 \mathrm{H}), 6.85(\mathrm{~d}, J=8.4 \mathrm{~Hz}, 1 \mathrm{H}), 5.59$ 
(ddd, $J=16.8,10.4,5.2 \mathrm{~Hz}, 1 \mathrm{H}), 5.16$ (d, $J=10.4 \mathrm{~Hz}, 1 \mathrm{H}), 4.79$ (d, $J=14.4 \mathrm{~Hz}, 1 \mathrm{H})$, 4.77-4.75 (m, 2H), $4.63(\mathrm{~d}, J=17.2 \mathrm{~Hz}, 1 \mathrm{H}), 4.43(\mathrm{~d}, J=14.4 \mathrm{~Hz}, 1 \mathrm{H}), 3.84$ (dd, $J=$ 12.8, $4.4 \mathrm{~Hz}, 1 \mathrm{H}), 3.79(\mathrm{~s}, 3 \mathrm{H}), 3.33(\mathrm{~d}, J=12.8 \mathrm{~Hz}, 1 \mathrm{H}) .{ }^{13} \mathrm{C}$ NMR $(100 \mathrm{MHz}$, $\left.\mathrm{CDCl}_{3}\right) \delta 159.1,157.3,133.0,129.8,128.0,125.5,118.5,115.6,113.9,106.5,100.7$, 55.4, 55.2, 48.63, 48.60. IR (thin film): $v_{\max }\left(\mathrm{cm}^{-1}\right)=3032,2960,2835,1639,1543$, 1418, 1242, 809, 740; HRMS (ESI) calcd for $\mathrm{C}_{17} \mathrm{H}_{17} \mathrm{Br}_{2} \mathrm{~N}_{2} \mathrm{O}_{2}[\mathrm{M}+\mathrm{H}]^{+}: 438.9651$. Found: 438.9641.<smiles>C=CC1CN(Cc2ccc(OC)cc2OC)C(=O)c2ccc(Br)n21</smiles>

(R)-31 ${ }^{4}$. Colourless oil, $67.6 \mathrm{mg}, 85 \%$ yield, 91\% ee [Daicel Chiralpak AD-H $(0.46 \mathrm{~cm} \mathrm{x} 25 \mathrm{~cm}), n$-hexane/2-propanol $=90 / 10, v=0.6 \mathrm{~mL} \cdot \min ^{-1}, \lambda=230 \mathrm{~nm}, \mathrm{t}$ $($ minor $)=52.99 \min , \mathrm{t}($ major $)=56.87 \mathrm{~min}] ;[\alpha]_{\mathrm{D}}{ }^{27}=+53.1\left(\mathrm{c}=1.0, \mathrm{CH}_{2} \mathrm{Cl}_{2}\right) .{ }^{1} \mathrm{H}$ NMR (400 MHz, $\left.\mathrm{CDCl}_{3}\right) \delta 7.21(\mathrm{~d}, J=6.0,3.2 \mathrm{~Hz}, 1 \mathrm{H}), 6.92(\mathrm{~d}, J=4.0 \mathrm{~Hz}, 1 \mathrm{H})$, 6.41-6.39 (m, 2H), $6.24(\mathrm{~d}, J=4.0 \mathrm{~Hz}, 1 \mathrm{H}), 5.61$ (ddd, $J=15.2,10.4,4.8 \mathrm{~Hz}, 1 \mathrm{H})$, $5.08(\mathrm{~d}, J=10.4 \mathrm{~Hz}, 1 \mathrm{H}), 4.76-4.72(\mathrm{~m}, 2 \mathrm{H}), 4.57(\mathrm{dd}, J=16.8,1.2 \mathrm{~Hz}, 1 \mathrm{H}), 4.48(\mathrm{~d}$, $J=14.4 \mathrm{~Hz}, 1 \mathrm{H}), 3.85(\mathrm{dd}, J=13.2,4.4 \mathrm{~Hz}, 1 \mathrm{H}), 3.76(\mathrm{~s}, 6 \mathrm{H}), 3.46(\mathrm{dd}, J=13.2,1.2$ $\mathrm{Hz}, 1 \mathrm{H})$. 
Figure S1. X-Ray crystal structure of $\mathbf{3 a}^{5}$

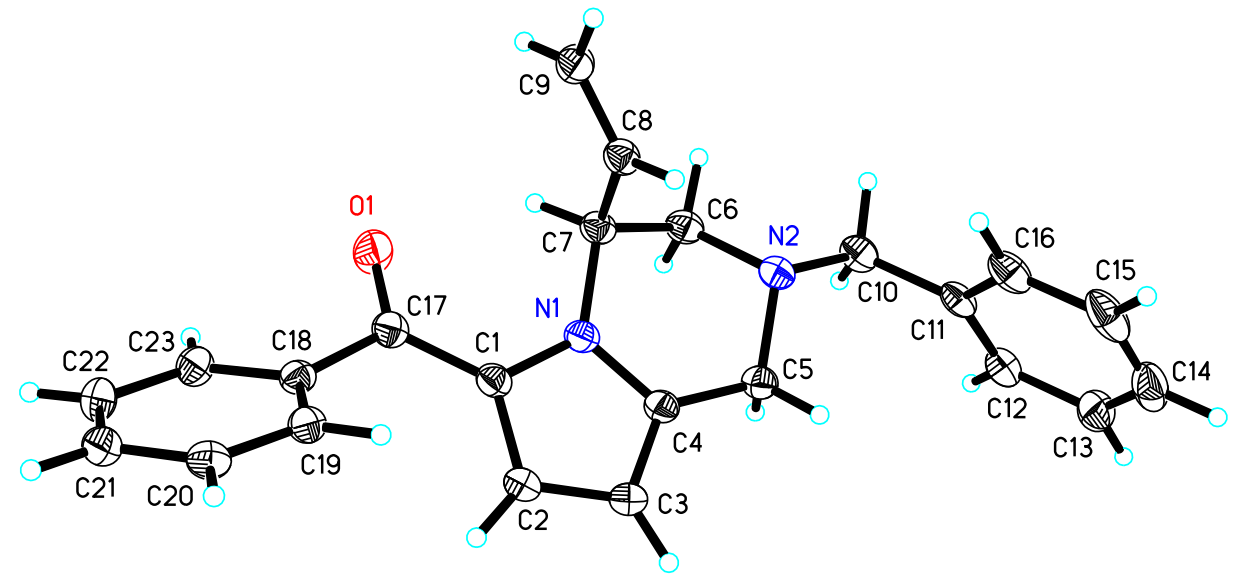

Table 1. Crystal data and structure refinement for cu_dm12359_0m.

Identification code

Empirical formula

Formula weight

Temperature

Wavelength

Crystal system, space group

Unit cell dimensions

Volume

Z, Calculated density

Absorption coefficient

$\mathrm{F}(000)$

Crystal size

Theta range for data collection

Limiting indices

Reflections collected / unique

Completeness to theta $=69.50$

Absorption correction

Max. and min. transmission

Refinement method

Data / restraints / parameters

Goodness-of-fit on $\mathrm{F}^{\wedge} 2$

Final R indices [I $>2 \operatorname{sigma}(\mathrm{I})]$

$\mathrm{R}$ indices (all data)

Absolute structure parameter cu_dm12359_0m

C23 H 22 N2 O

342.43

173(2) K

$1.54178 \mathrm{~A}$

Orthorhombic, P2(1)2(1)2(1)

$\mathrm{a}=8.92130(10)$ A $\quad$ alpha $=90 \mathrm{deg}$.

$\mathrm{b}=9.93250(10) \mathrm{A} \quad$ beta $=90 \mathrm{deg}$.

$\mathrm{c}=20.8095(3)$ A $\quad$ gamma $=90 \mathrm{deg}$.

1843.95(4) $\mathrm{A}^{\wedge} 3$

4, $\quad 1.233 \mathrm{Mg} / \mathrm{m}^{\wedge} 3$

$0.593 \mathrm{~mm}^{\wedge}-1$

728

$0.25 \times 0.20 \times 0.14 \mathrm{~mm}$

4.25 to $69.50 \mathrm{deg}$.

$-10<=\mathrm{h}<=10,-12<=\mathrm{k}<=11,-25<=1<=24$

$17113 / 3282$ [R(int) $=0.0287]$

$98.9 \%$

Semi-empirical from equivalents

0.9216 and 0.8660

Full-matrix least-squares on $\mathrm{F}^{\wedge} 2$

3282 / 1 / 236

1.030

$\mathrm{R} 1=0.0274, \mathrm{wR} 2=0.0980$

$\mathrm{R} 1=0.0274, \mathrm{wR} 2=0.0984$

$0.2(2)$ 
Extinction coefficient

$0.039(2)$

Largest diff. peak and hole

0.115 and -0.152 e. $\mathrm{A}^{\wedge}-3$

References:

(1) (a) Liu, W.-B.; He, H.; Dai, L.-X.; You, S.-L. Synthesis 2009, 2076-2082. (b) Liu, W.-B.; Zheng, C.; Zhuo, C.-X.; Dai, L.-X.; You, S.-L. J. Am. Chem. Soc. 2012, 134, 4812-4821.

(2) You, H. T.; Grosse, A. C.; Howard, J. K.; Hyland, C. J. T.; Just, J.; Molesworth, P. P.; Smith, J. A. Org. Biomol. Chem. 2011, 9, 3948-3953.

(3) Trost, B. M.; Sacchi, K. L.; Schroeder, G. M.; Asakawa, N. Org. Lett. 2002, 4, 3427-3430.

(4) Trost, B. M.; Osipov, M.; Dong, G. J. Am. Chem. Soc. 2010, 132, 15800-15807.

(5) Flack, H. D. Acta Crystallogr. 1983, A39, 876. 


\section{Copies of NMR Spectra}

NMR Spectra of $\mathbf{2 a}$

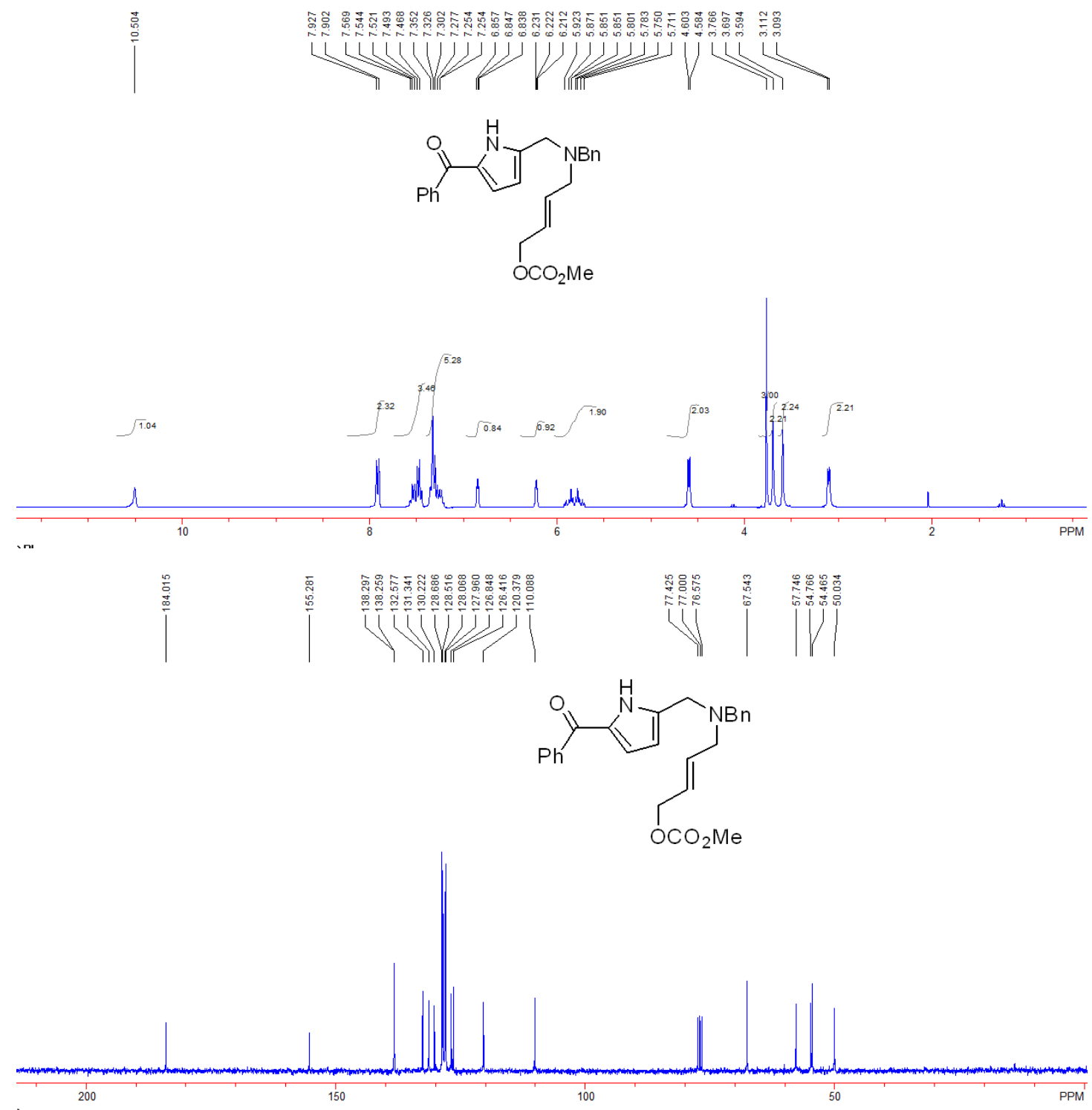

NMR Spectra of $\mathbf{2 b}$ 


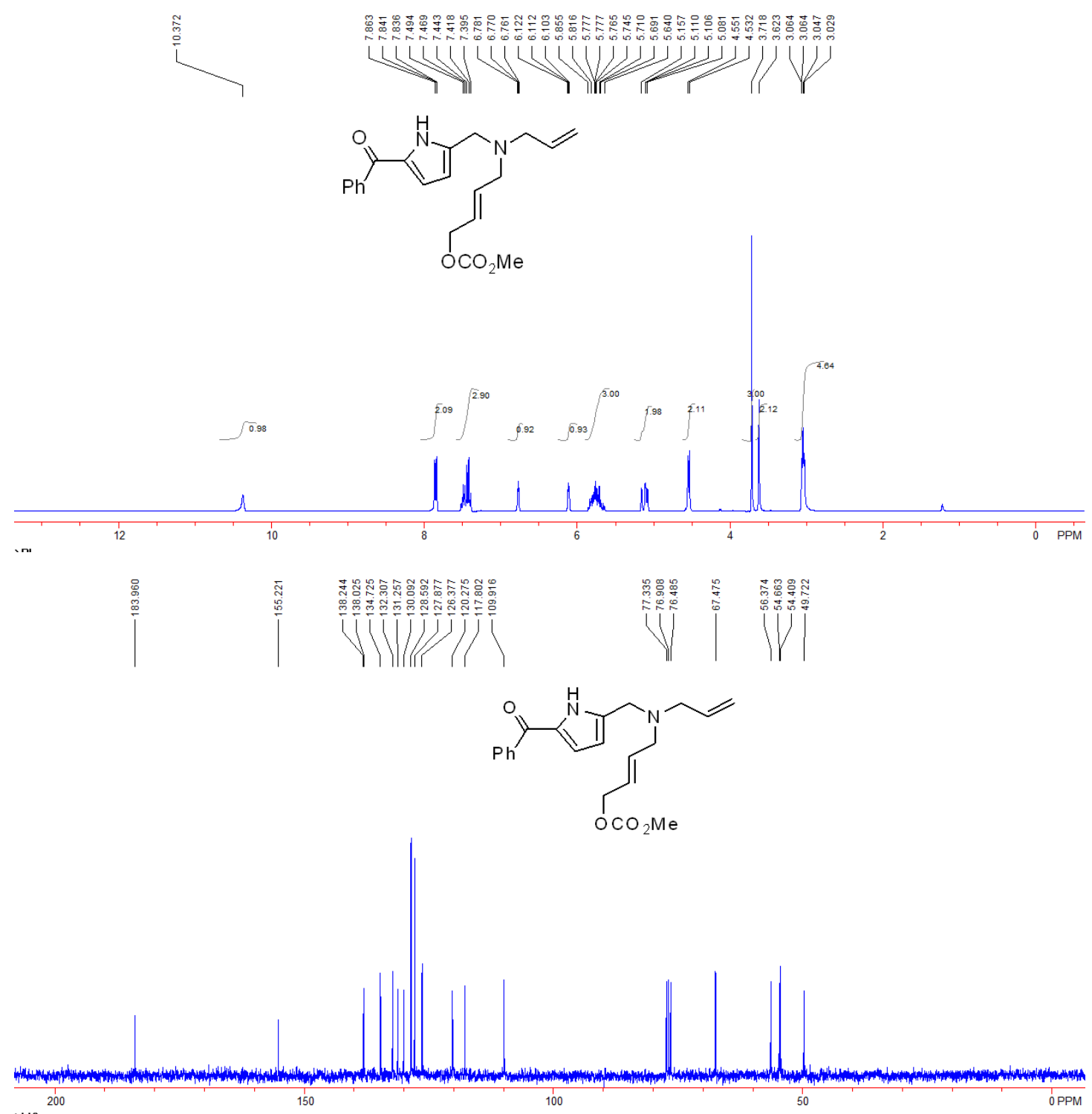

NMR Spectra of 2c

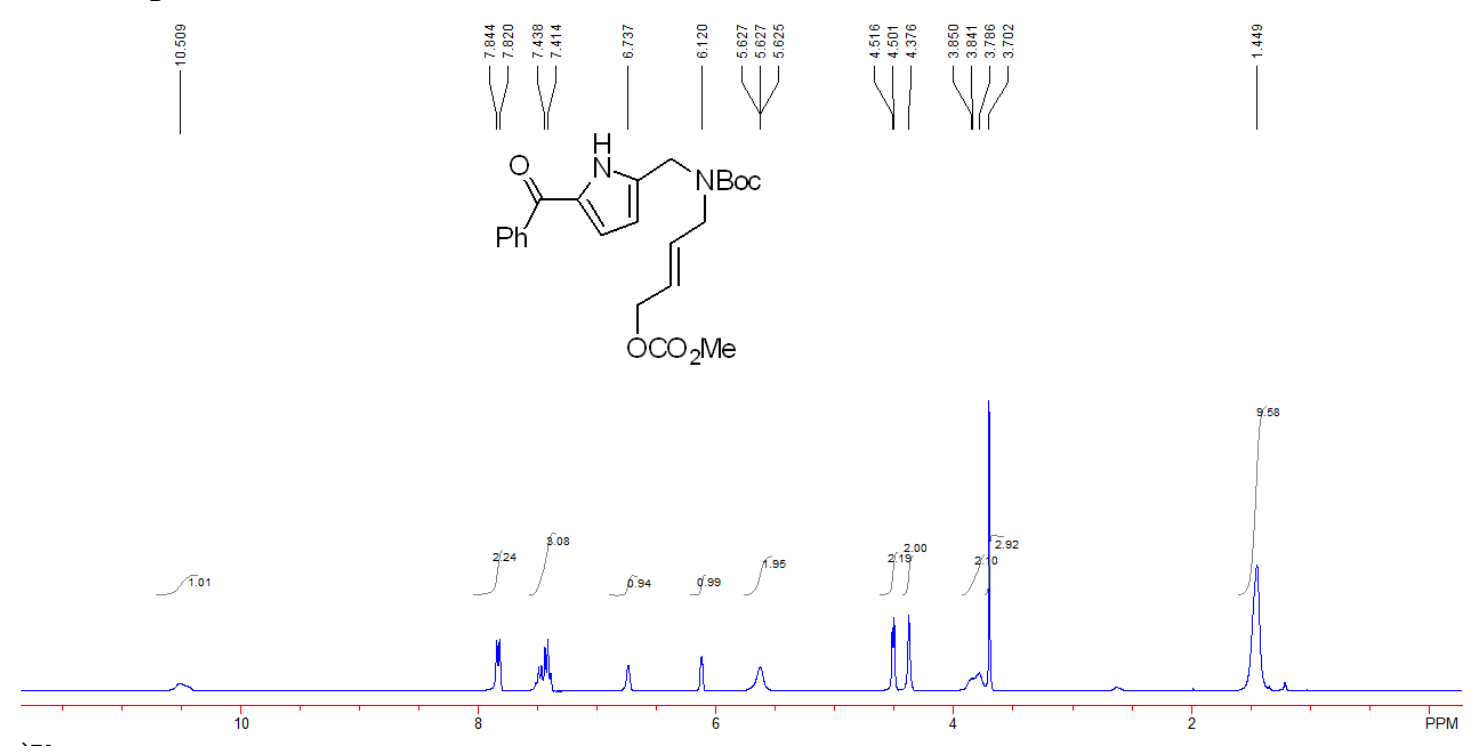




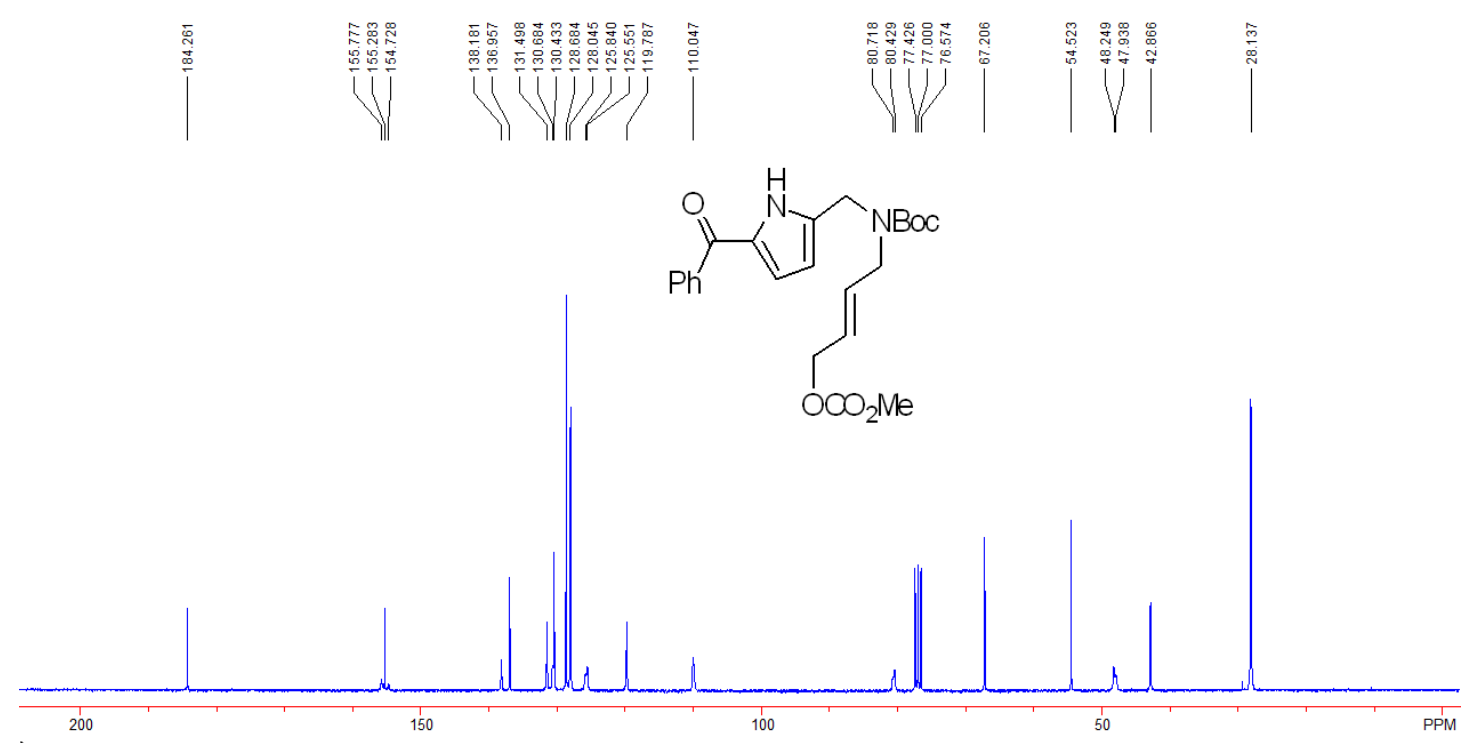

NMR Spectra of $\mathbf{2 d}$

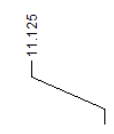

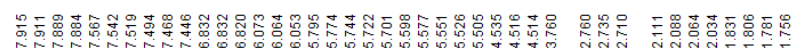

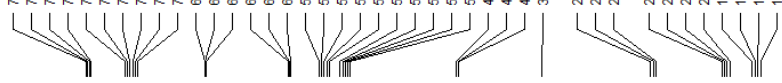<smiles>COC/C=C/CCCc1ccc(C(=O)c2ccccc2)[nH]1</smiles>

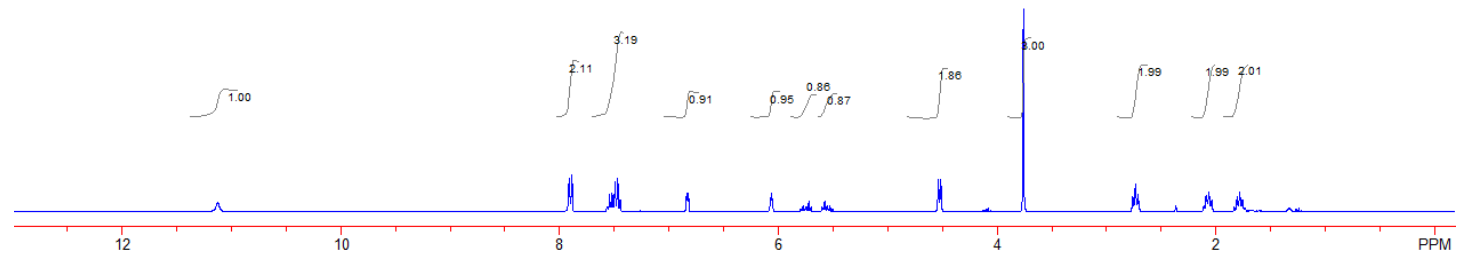

$\cdots$
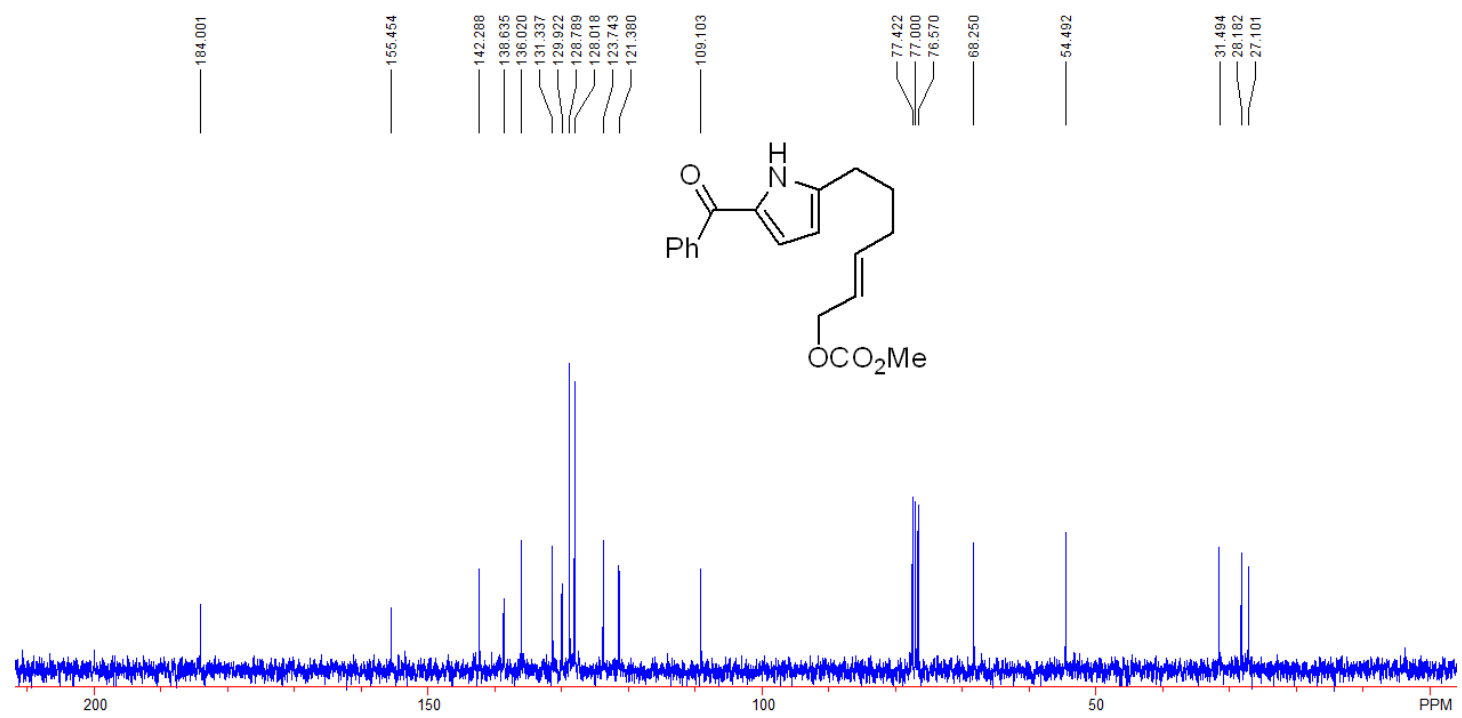

NMR Spectra of $\mathbf{2 e}$ 


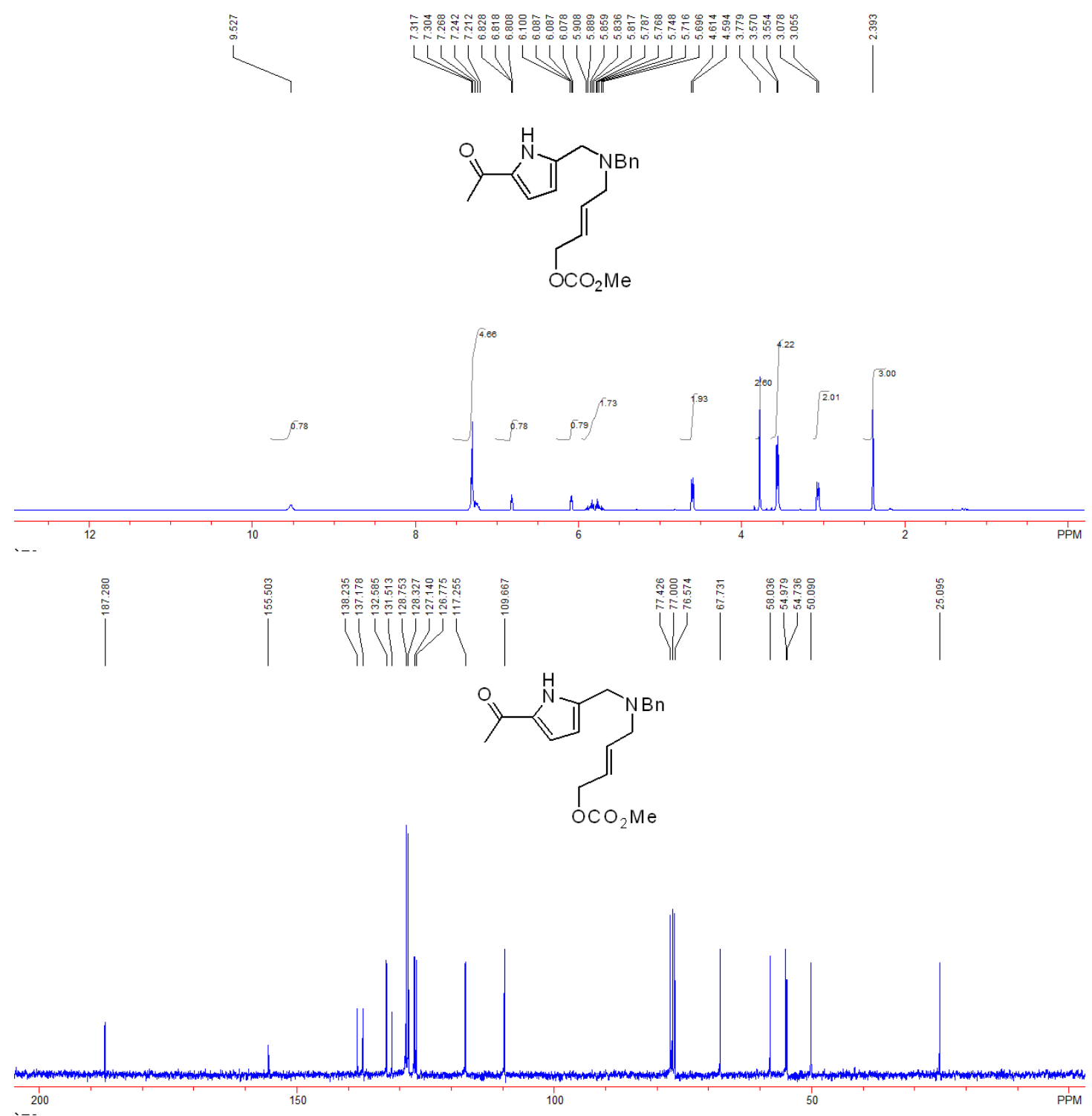

NMR Spectra of $\mathbf{2 f}$

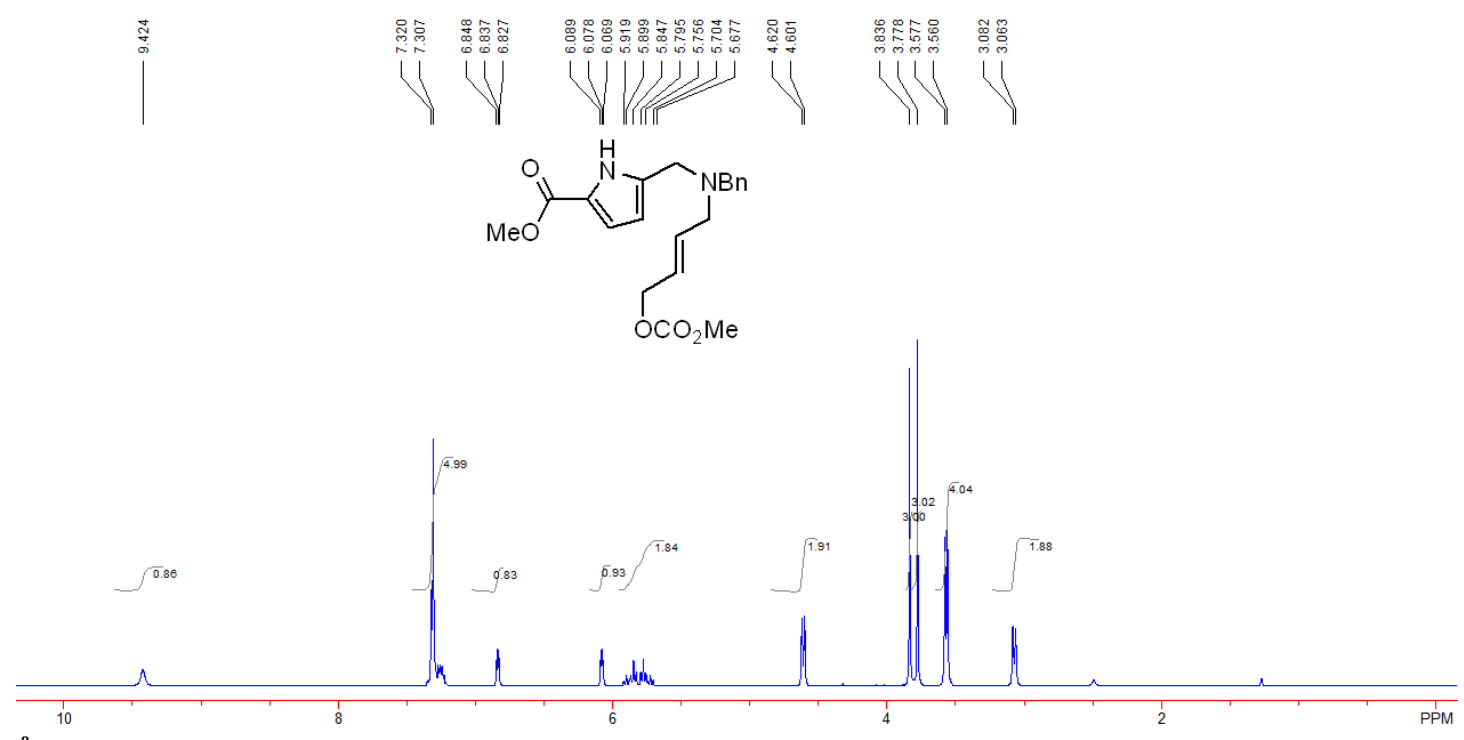



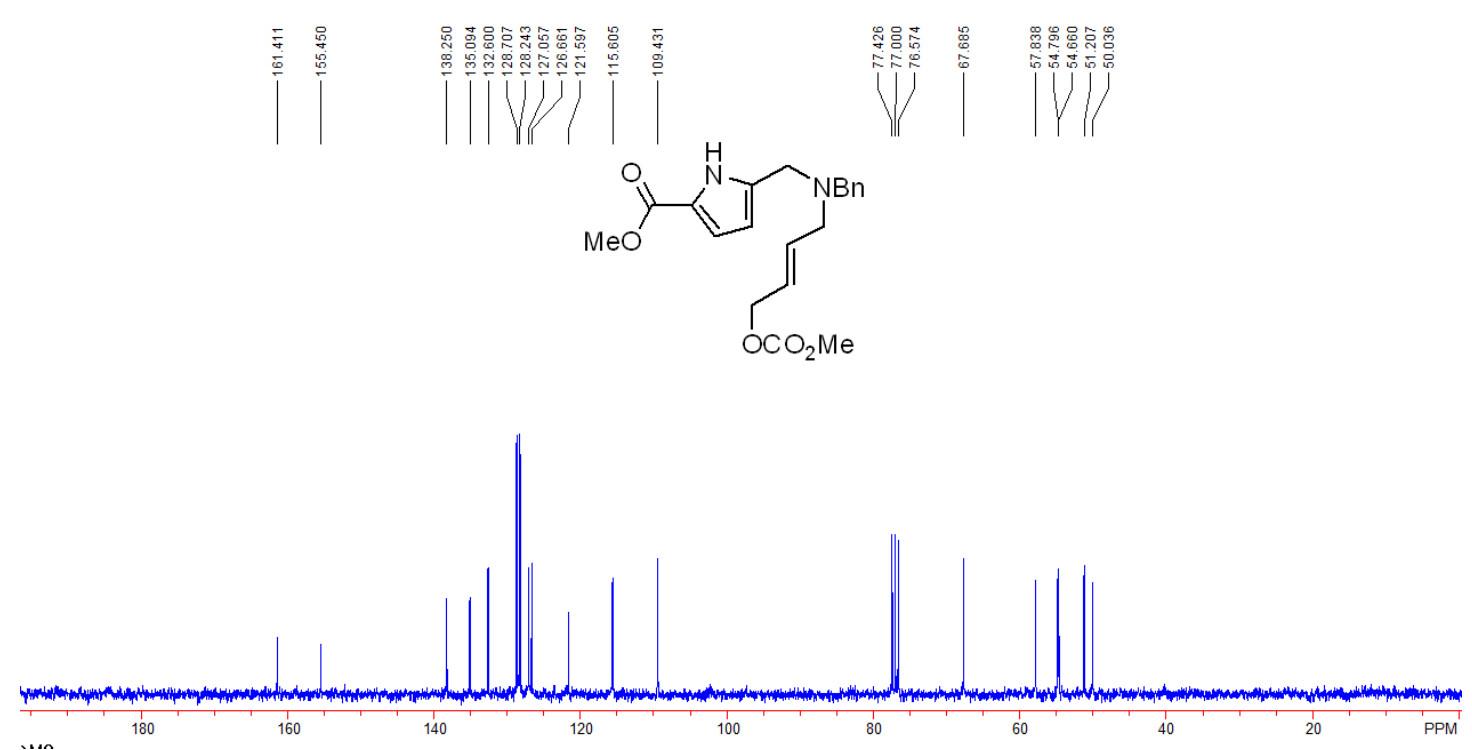

NMR Spectra of $\mathbf{2 g}$
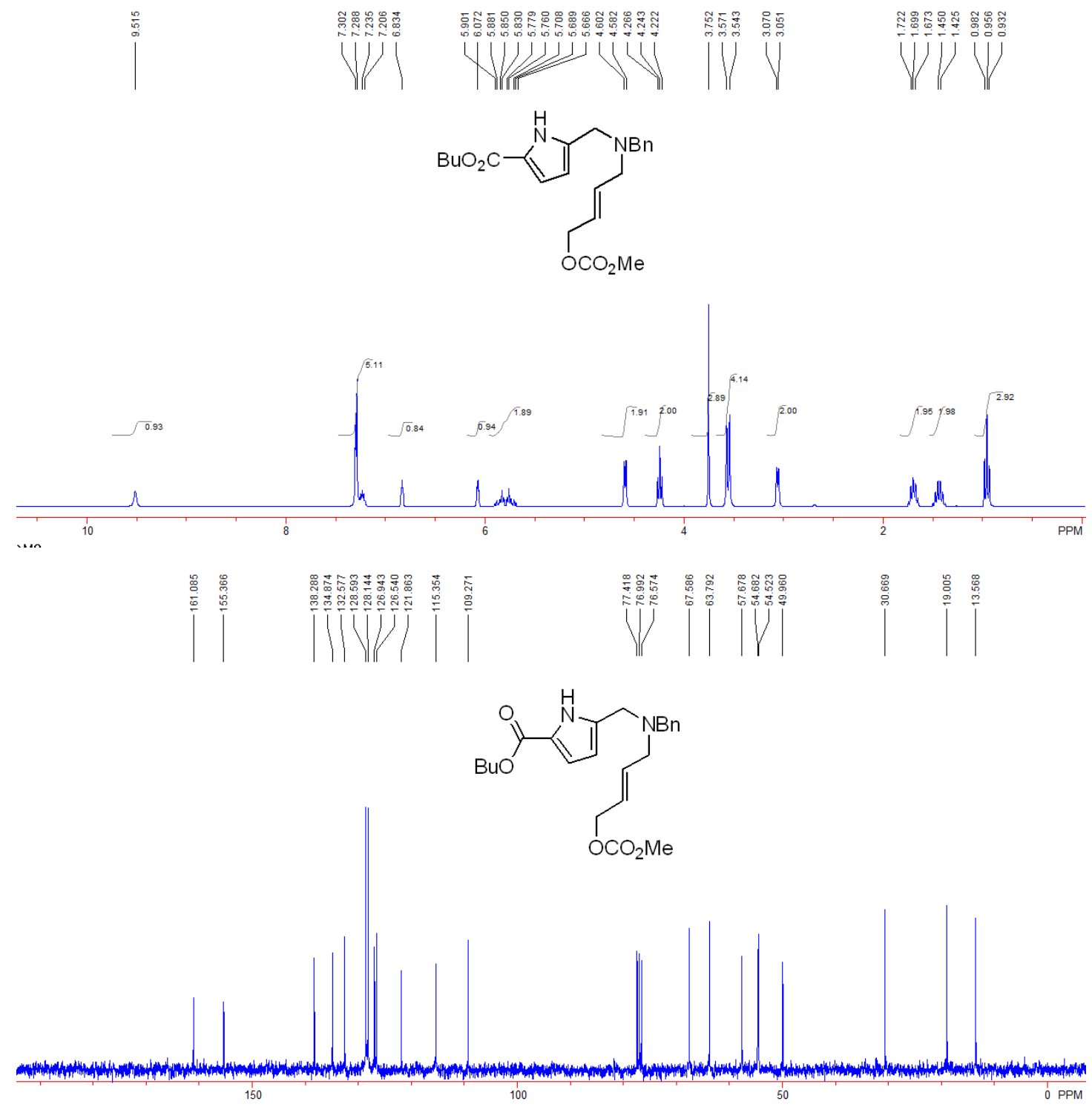

NMR Spectra of $\mathbf{2 h}$ 

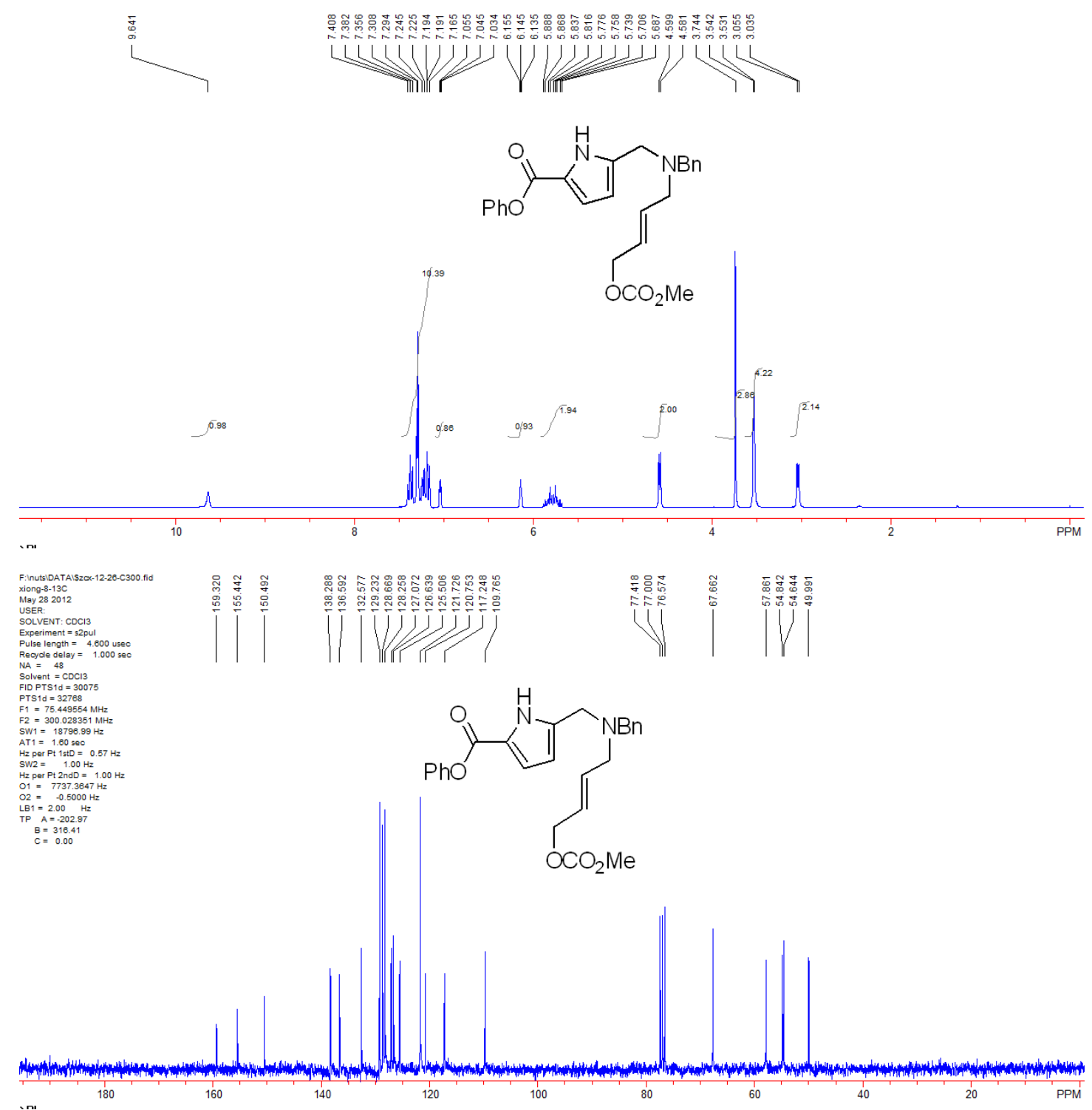

NMR Spectra of $\mathbf{2 i}$

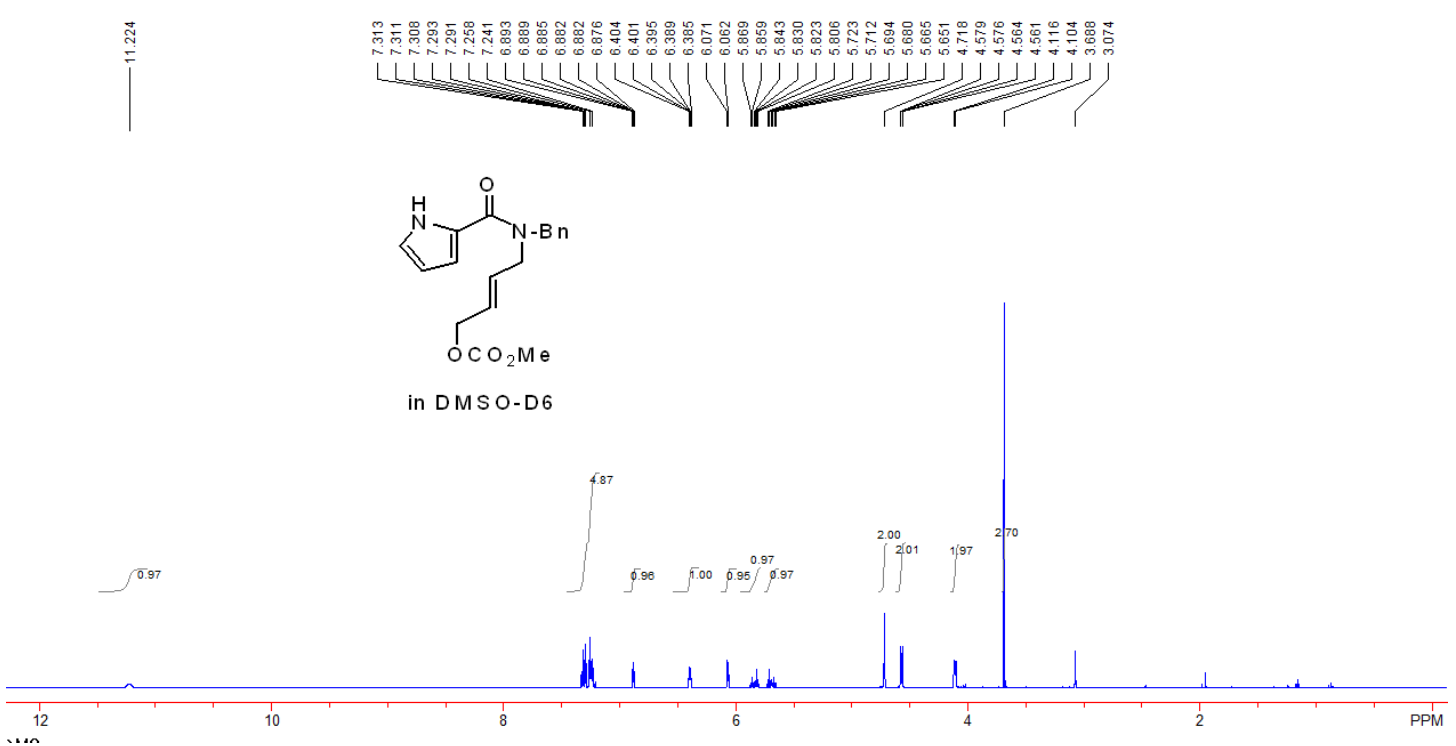




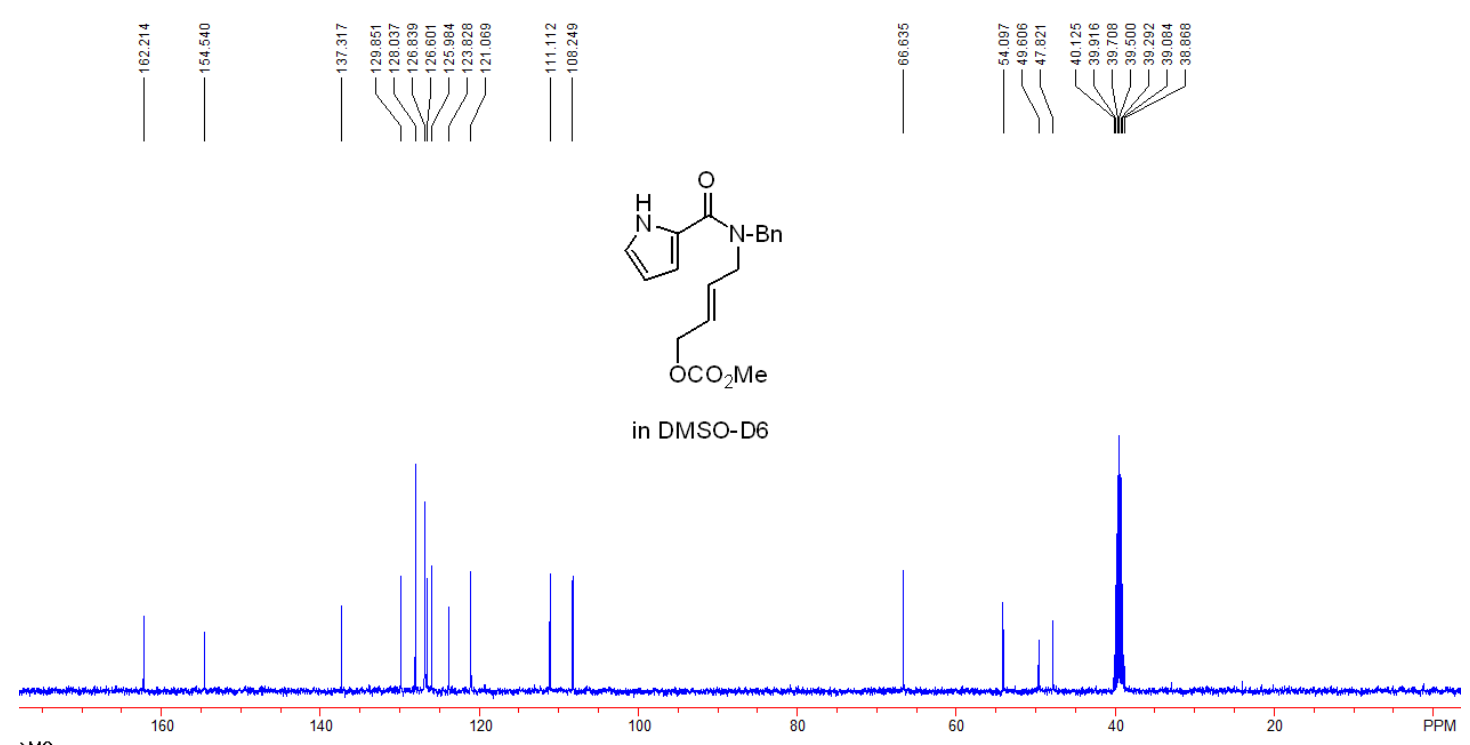

NMR Spectra of $\mathbf{2} \mathbf{j}$

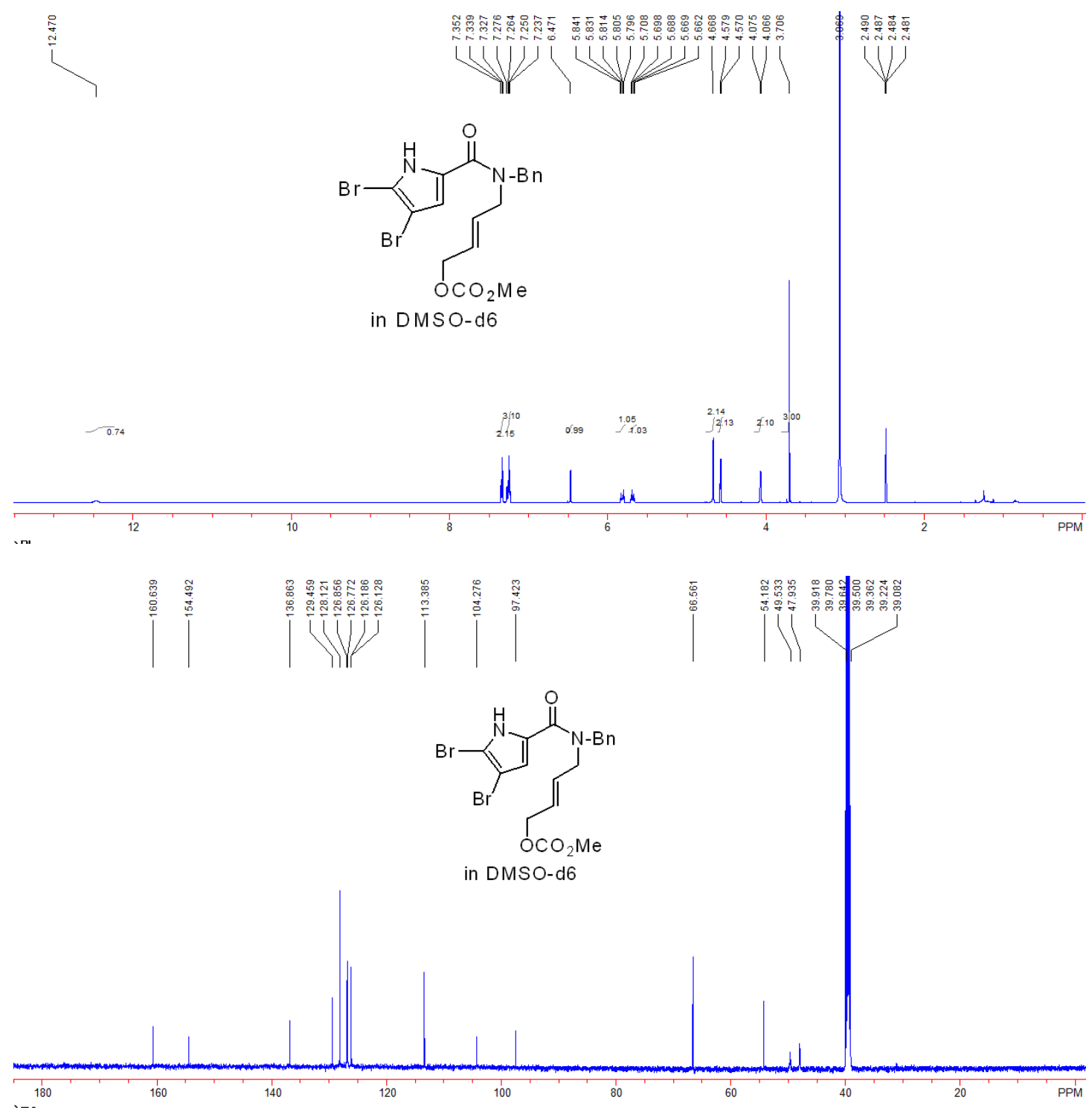

NMR Spectra of $\mathbf{2 k}$ 


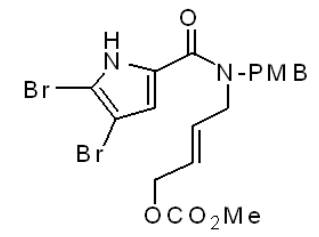

in $d 6-D M S O$
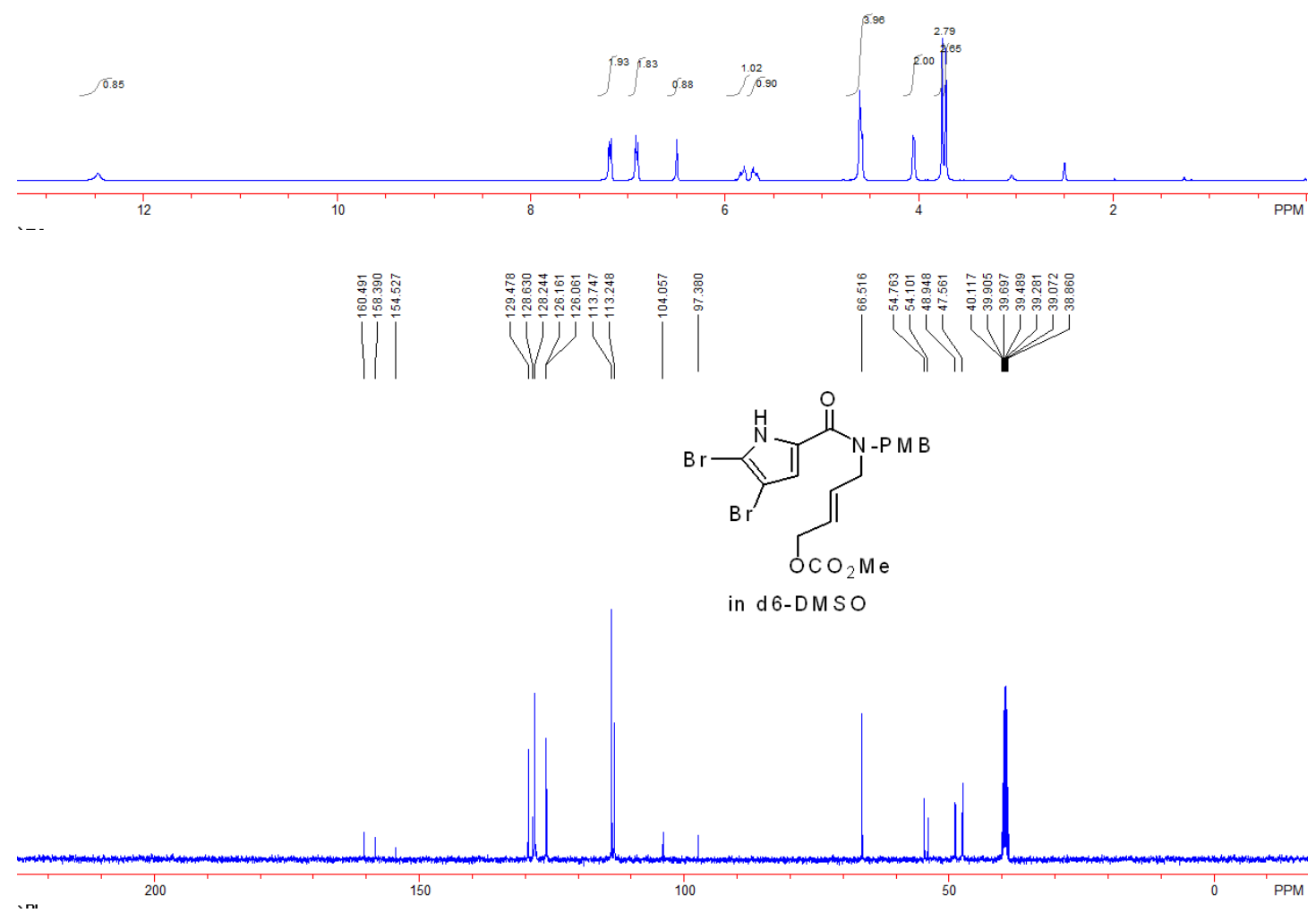

NMR Spectra of $2 \mathbf{I}$

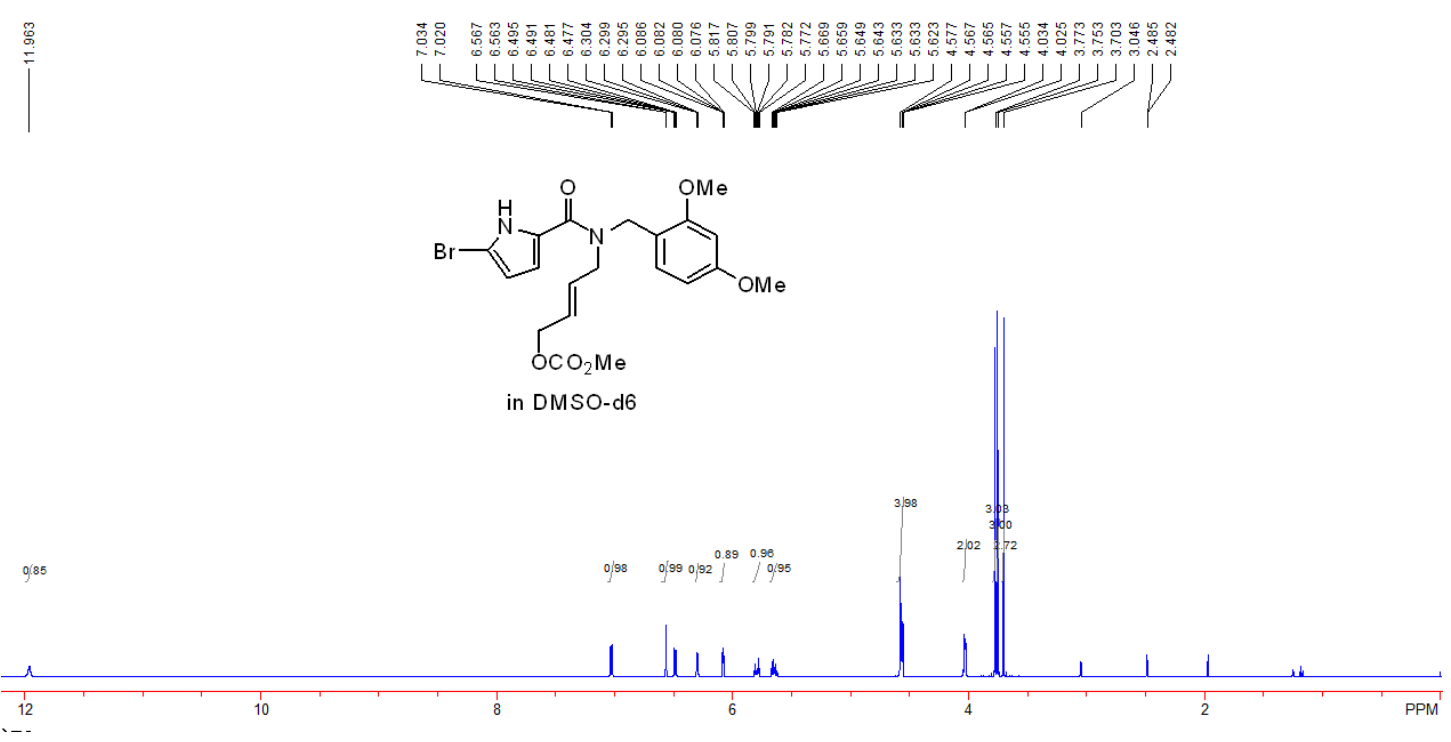




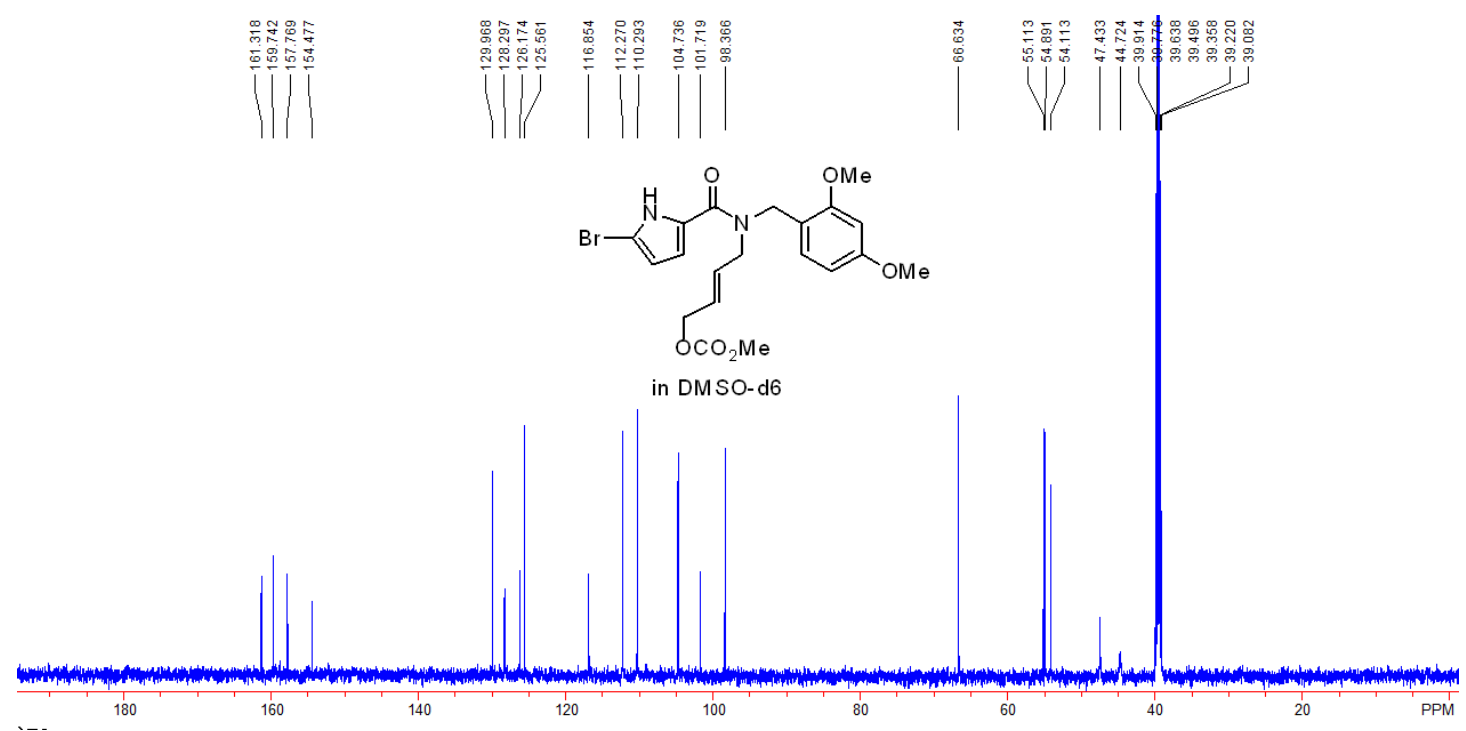

NMR Spectra of S5-1

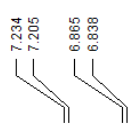

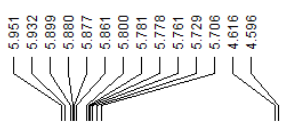

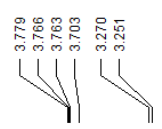

舟
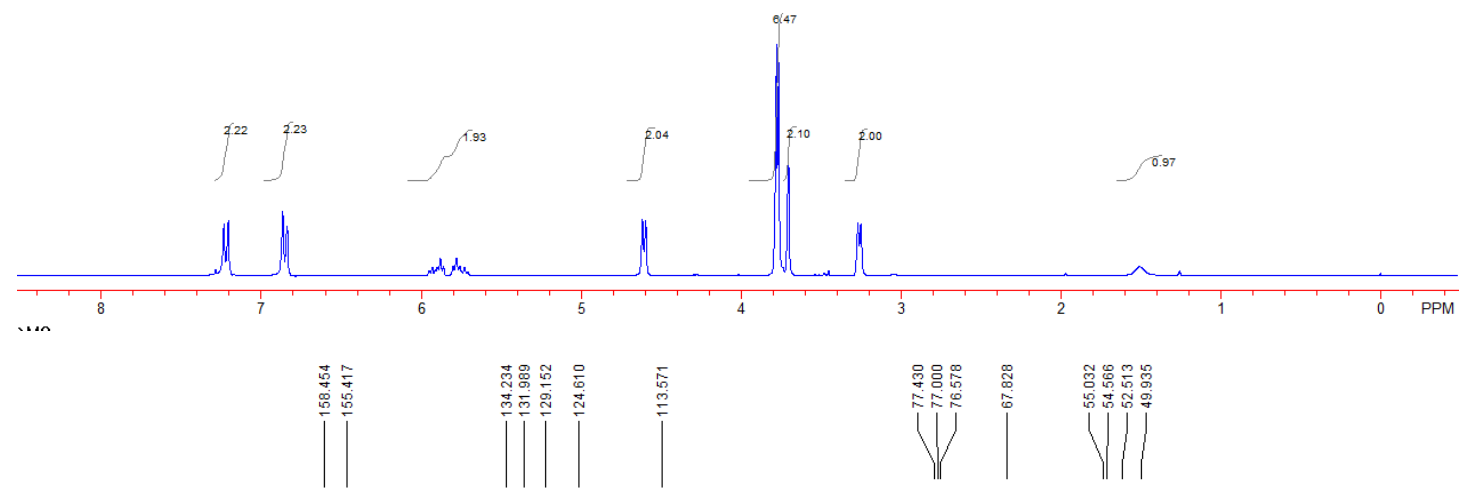

$\mathrm{MeO}_{2} \mathrm{CO} \leadsto \mathrm{N}$

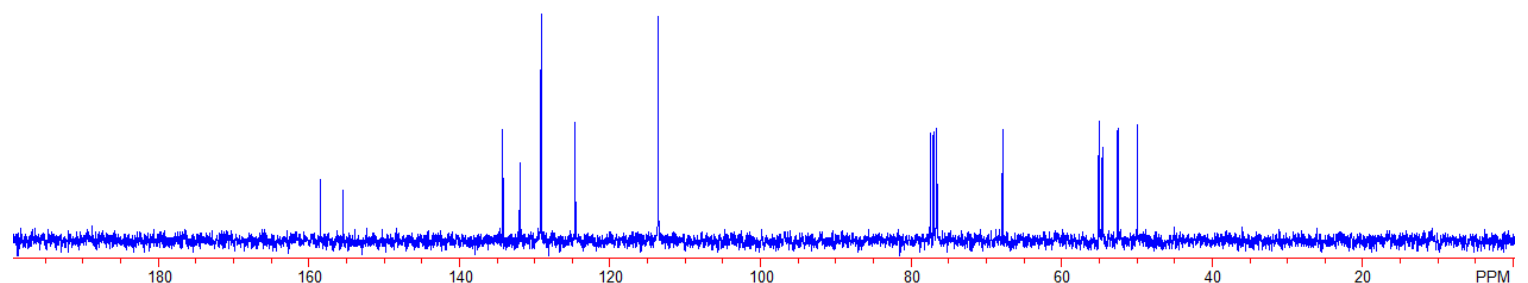

NMR Spectra of S5-2 


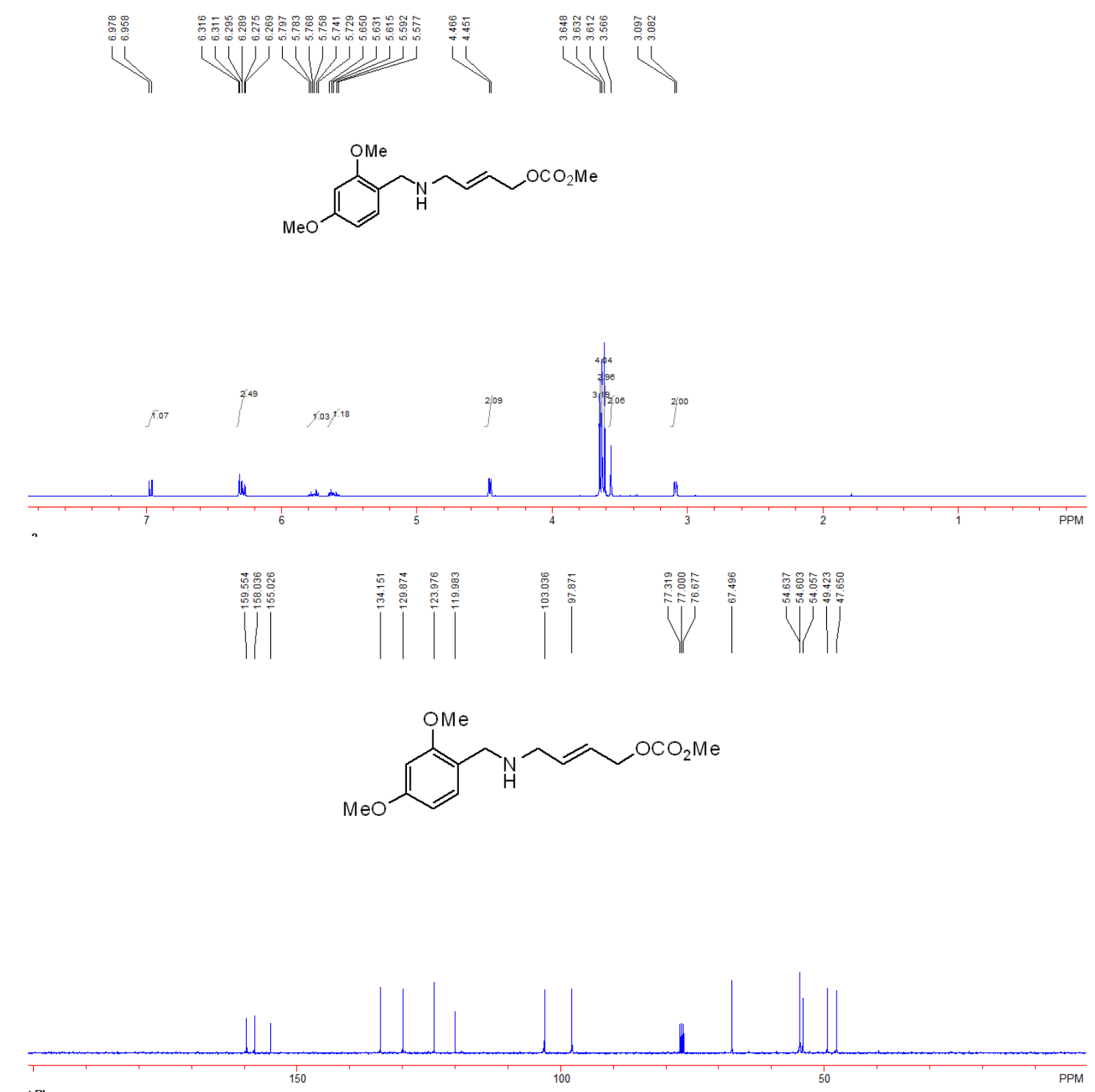

NMR Spectra of 3a

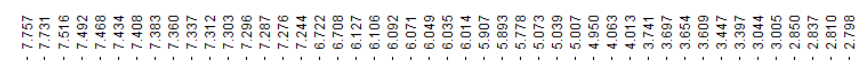

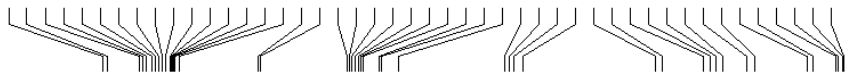

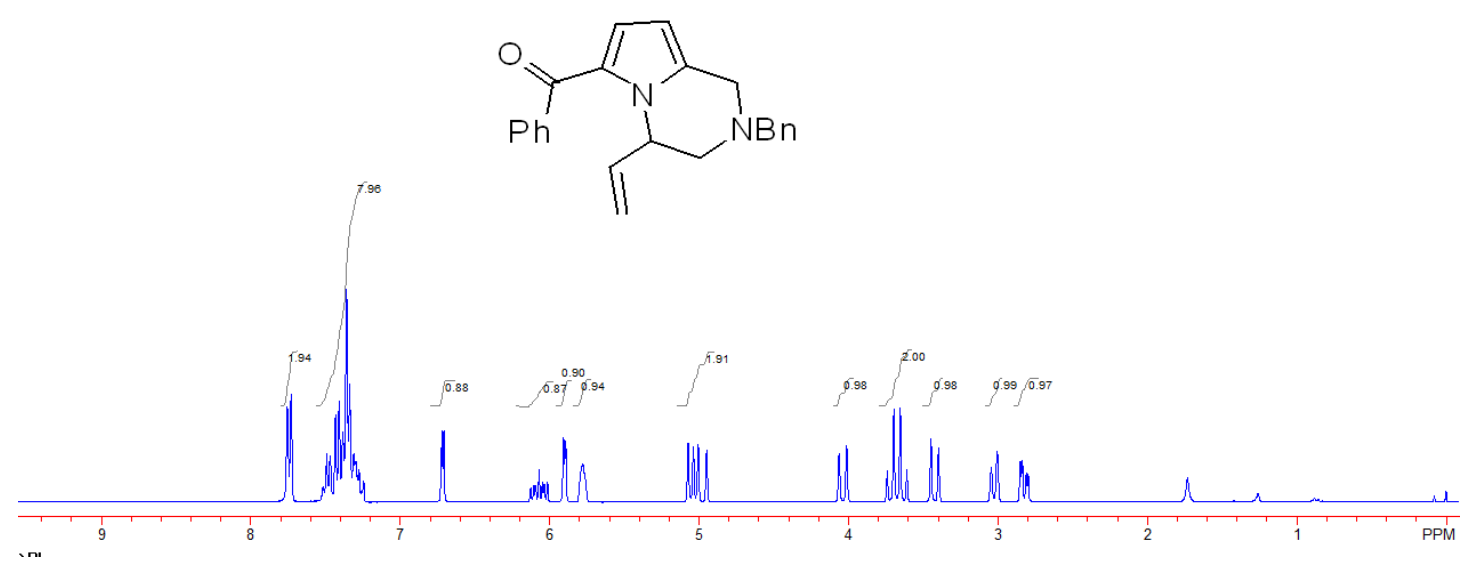




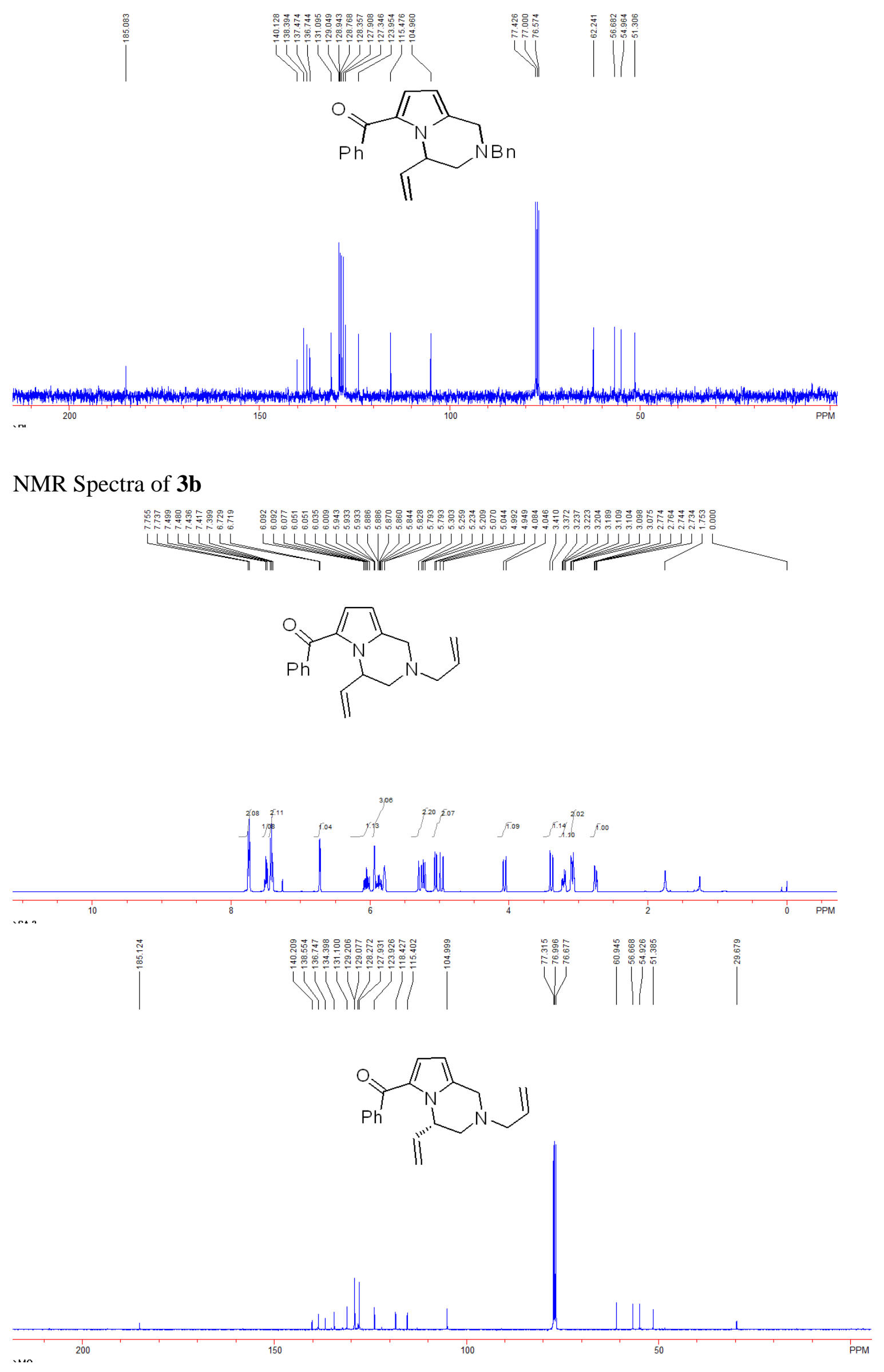


NMR Spectra of $\mathbf{3 c}$

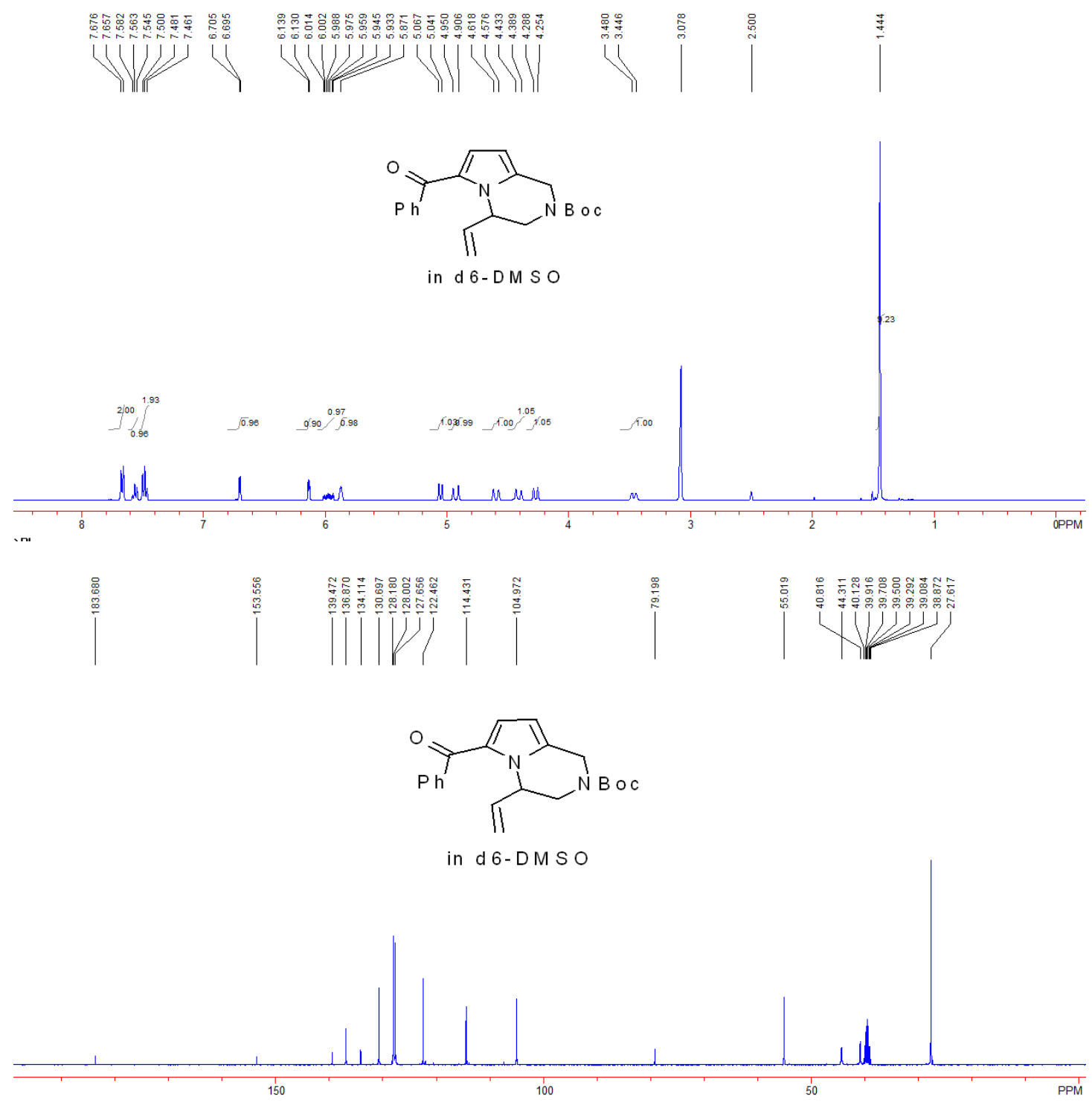

NMR Spectra of $\mathbf{3 d}$ 

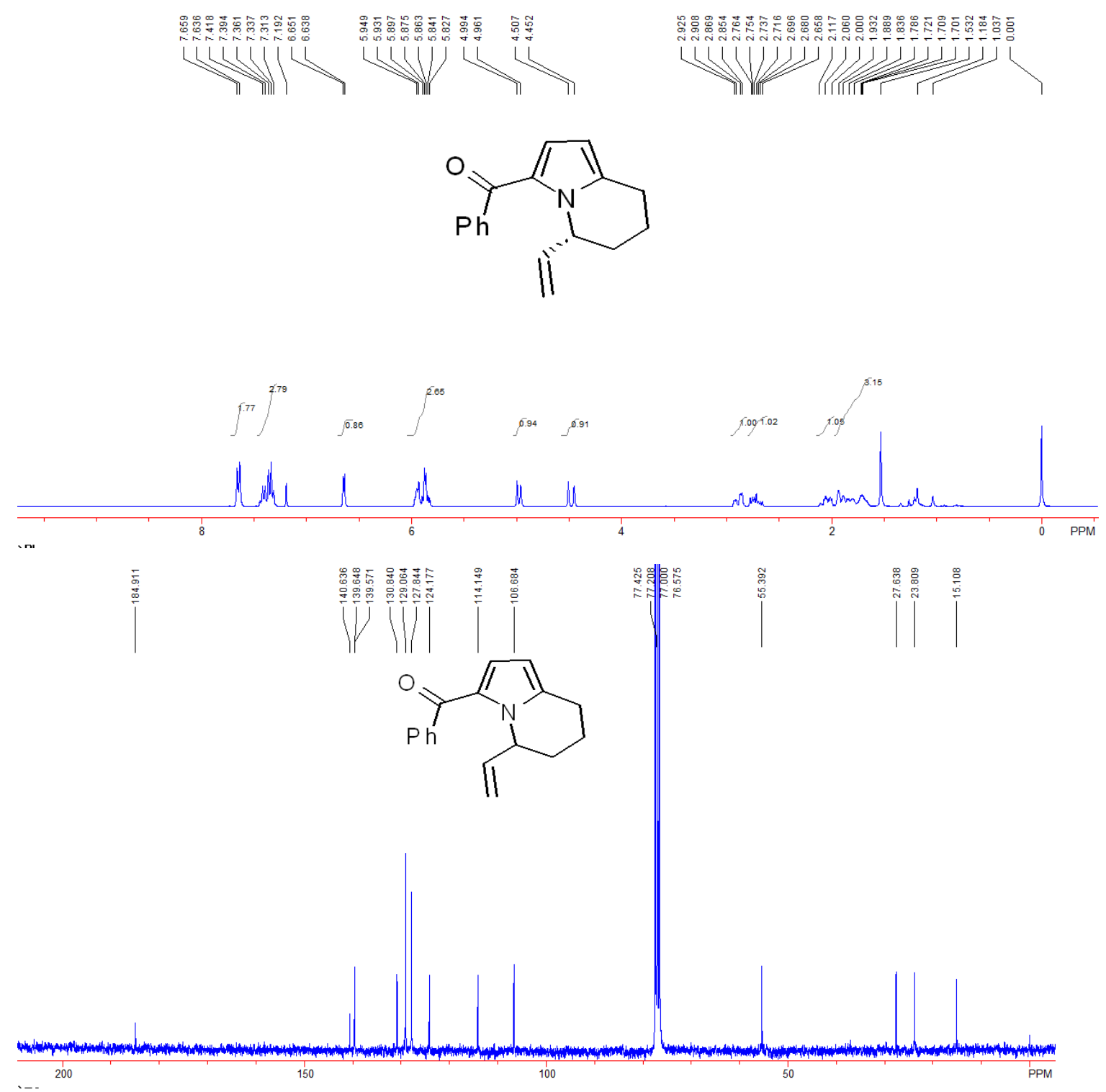

NMR Spectra of $3 \mathbf{e}$

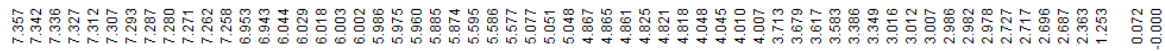

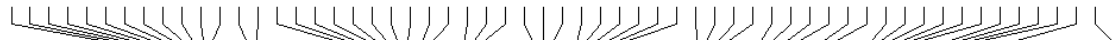

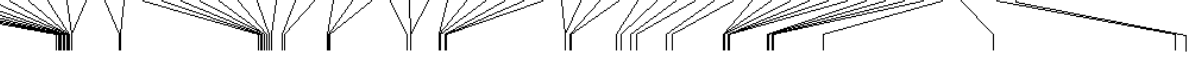<smiles>C=C[C@@H]1C[NH2+]Cc2ccc(C(C)=O)n21</smiles>

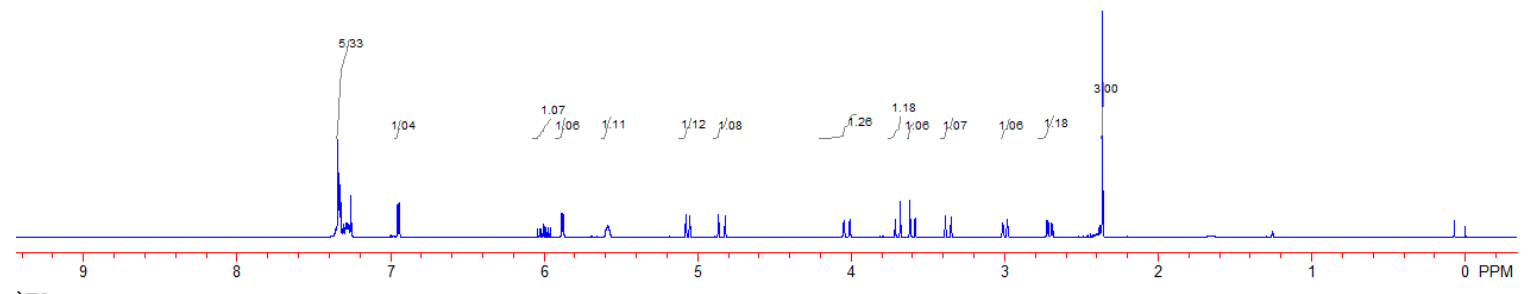




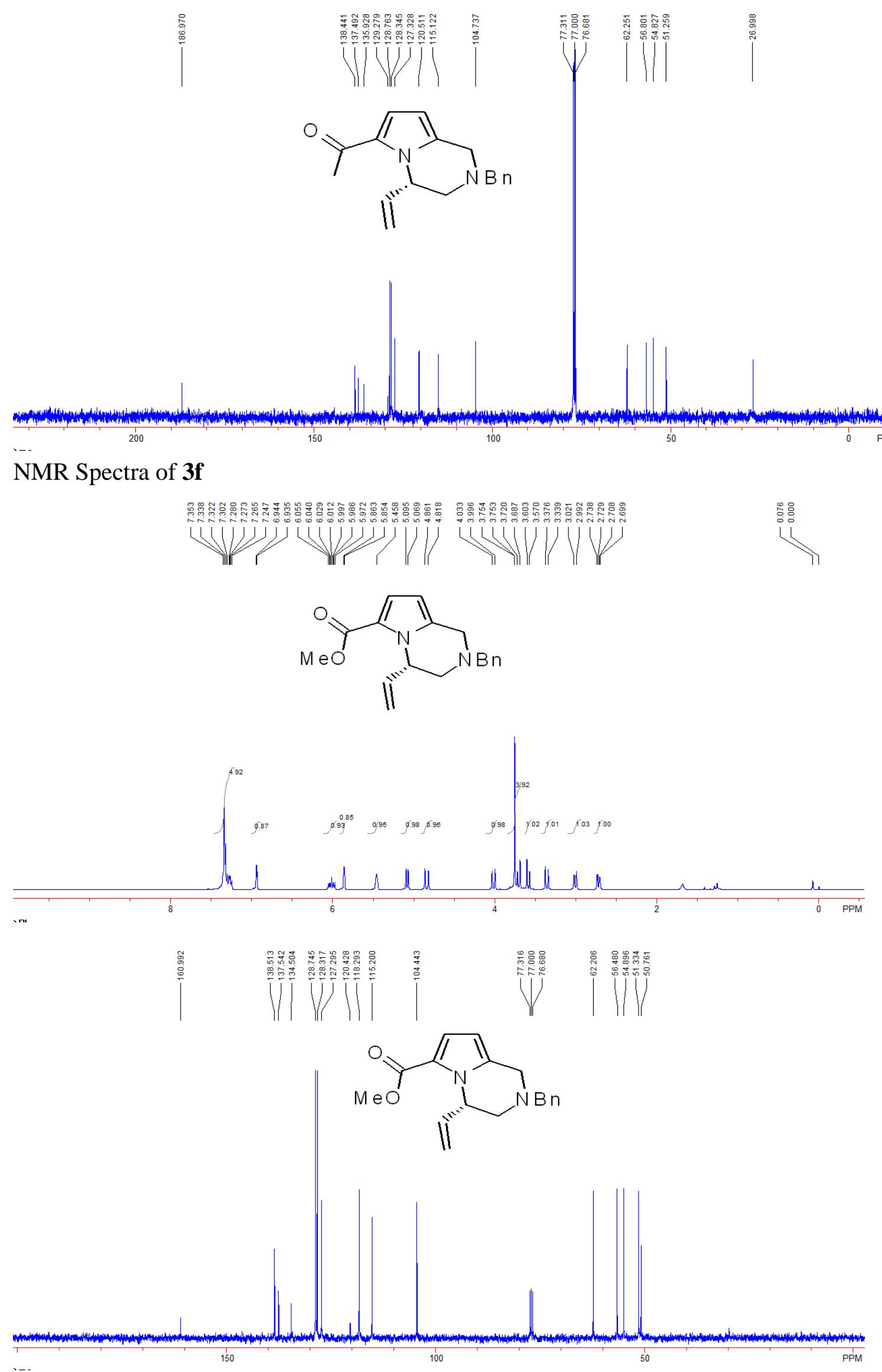

NMR Spectra of $\mathbf{3 g}$ 

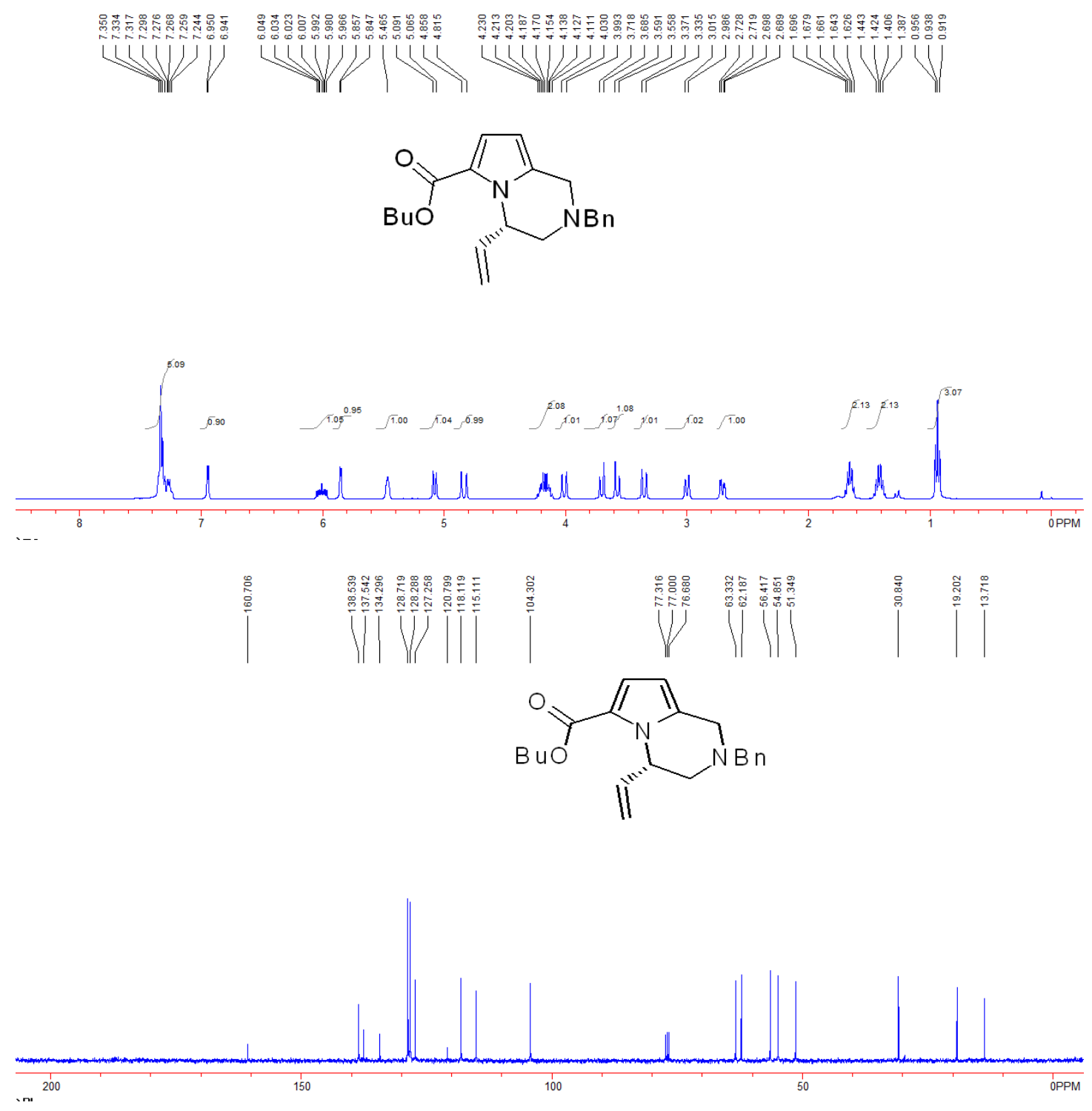

NMR Spectra of $\mathbf{3 h}$

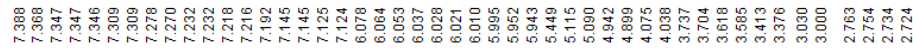

LuLU

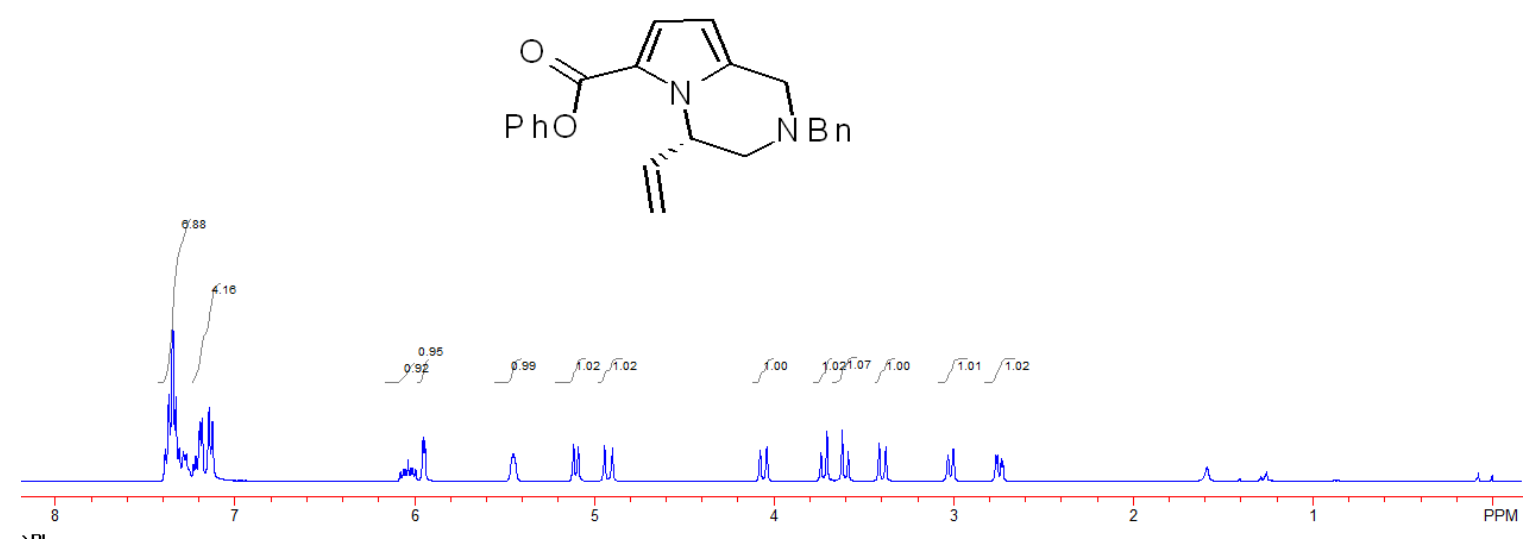



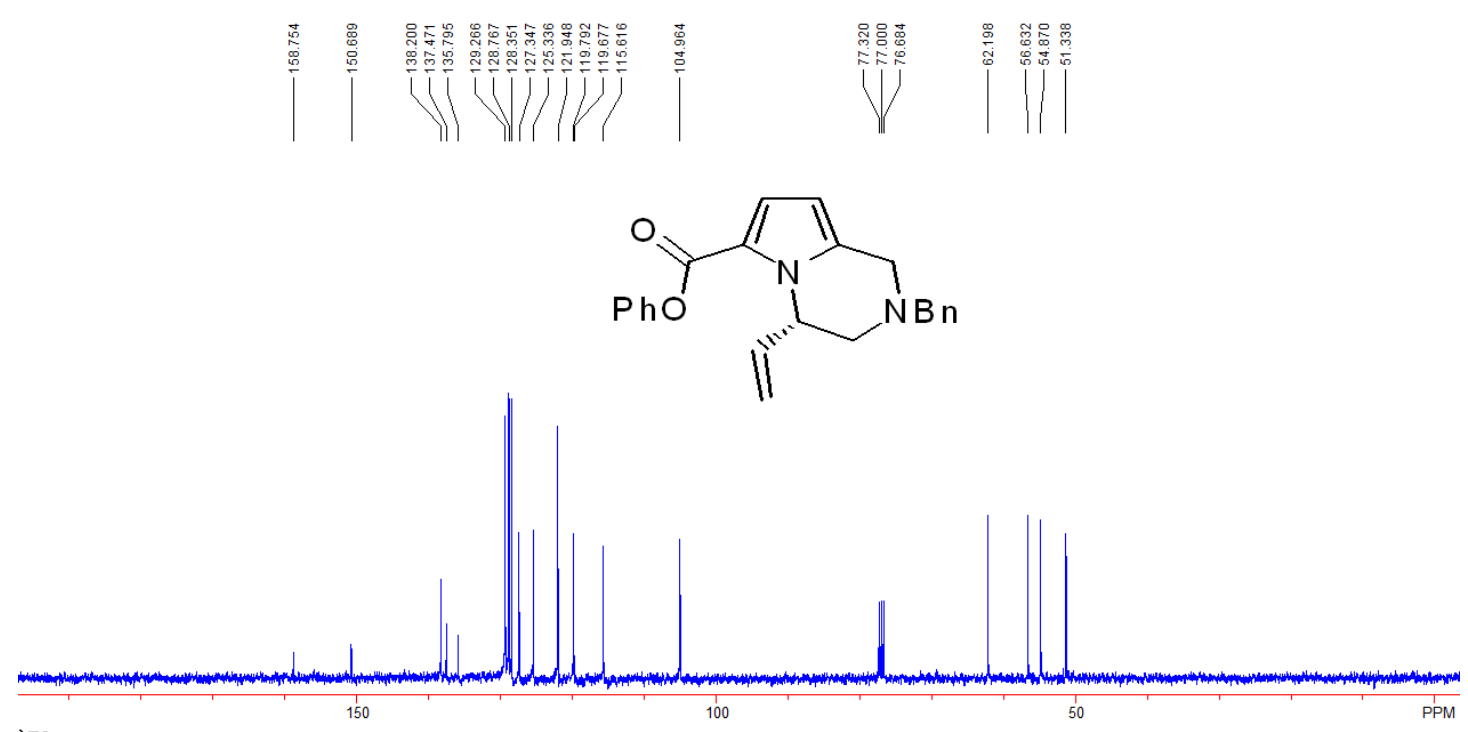

\section{NMR Spectra of $\mathbf{3 i}$}

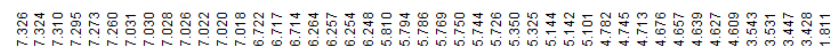

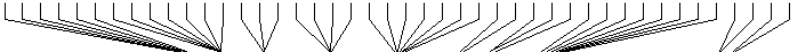
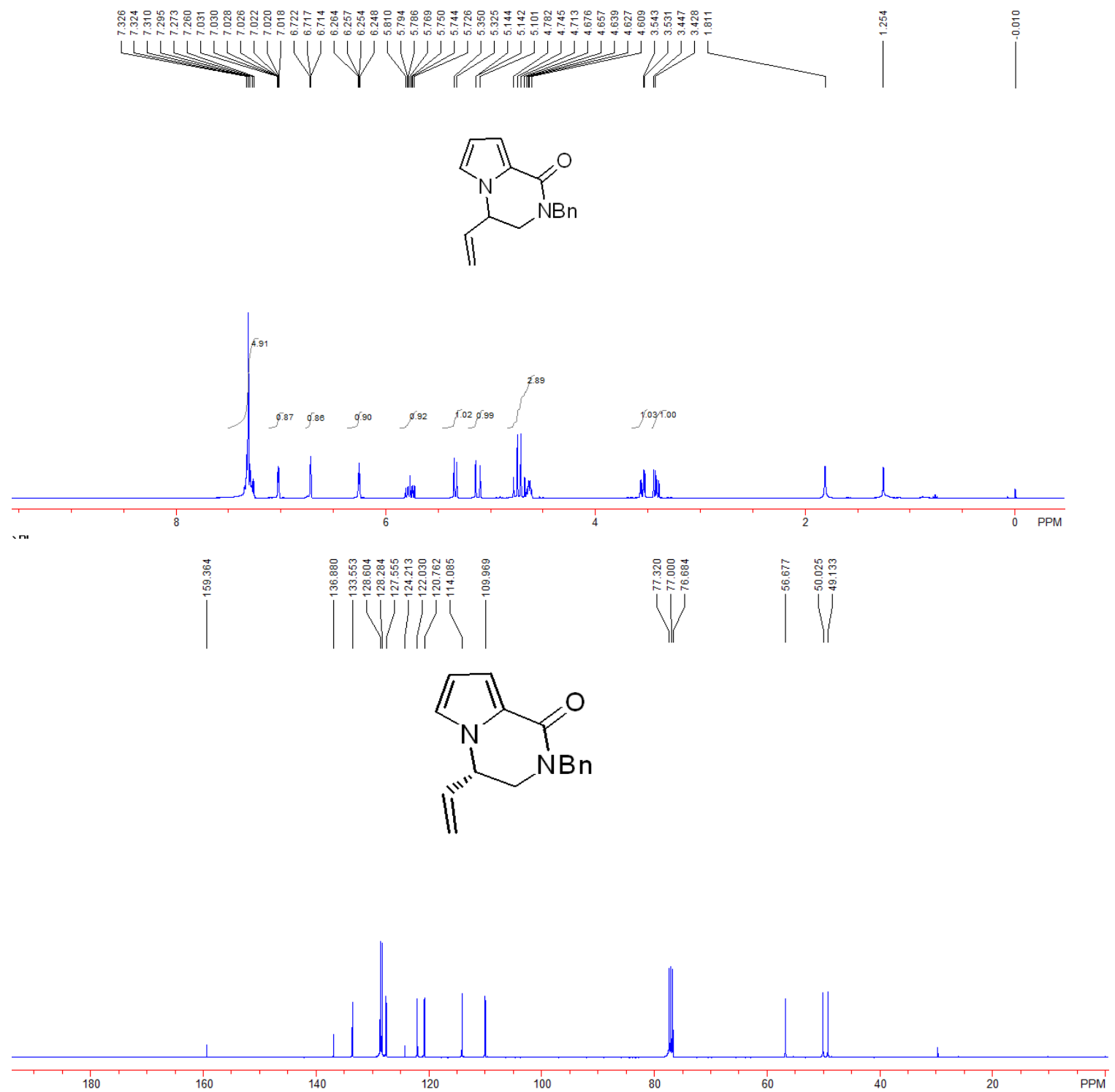

NMR Spectra of $\mathbf{3 j}$ 


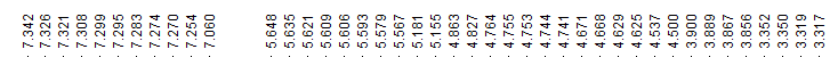

LU)<smiles>C=CC1CNC(=O)c2cc(Br)c(Br)n21</smiles>
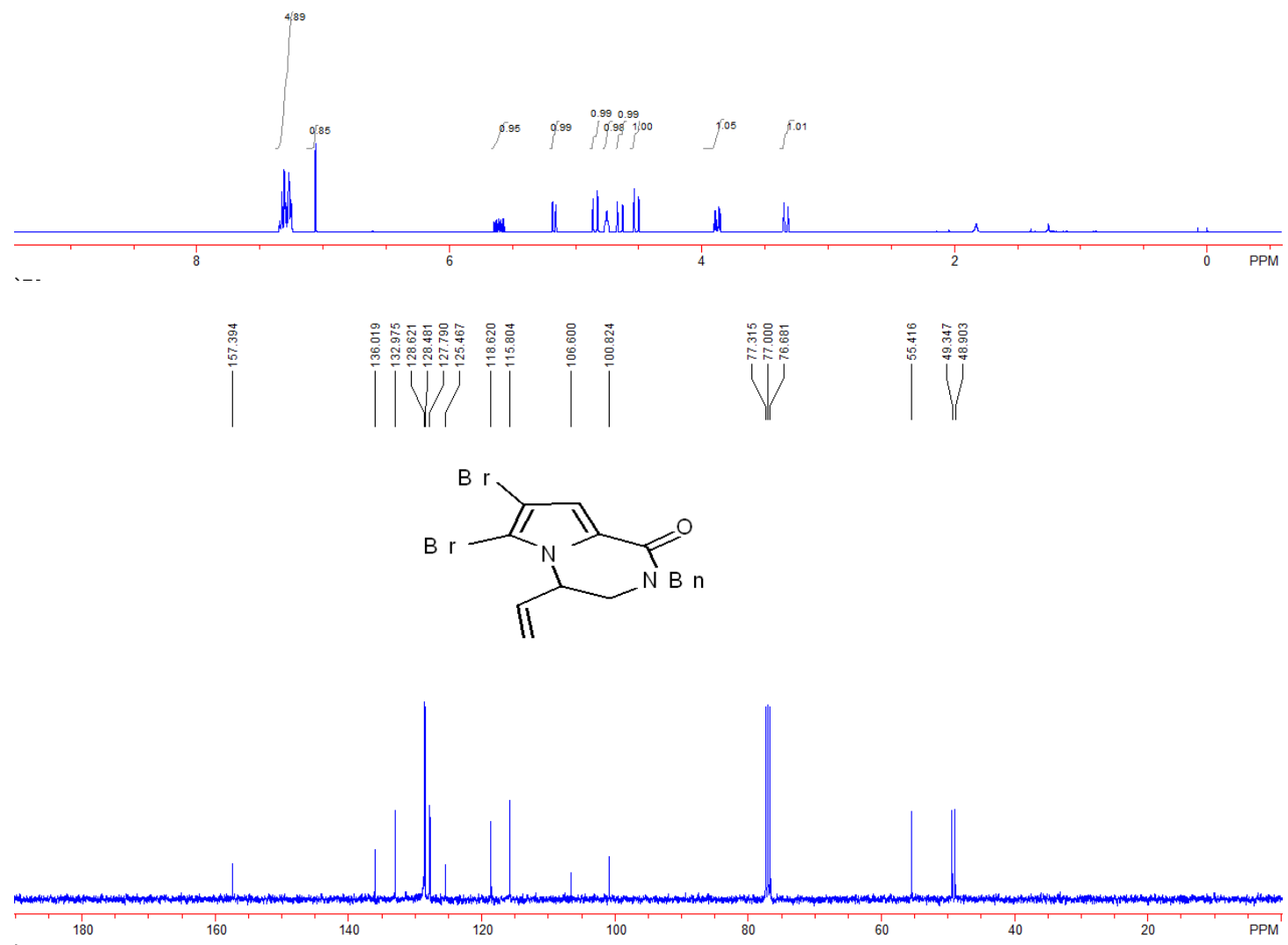

NMR Spectra of $\mathbf{3 k}$

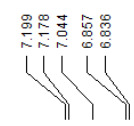

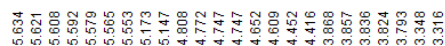

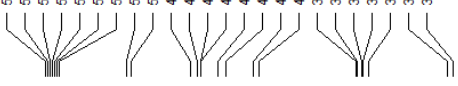<smiles>C=CC1CN(C)C(=O)c2cc(Br)c(Br)n2C1</smiles>

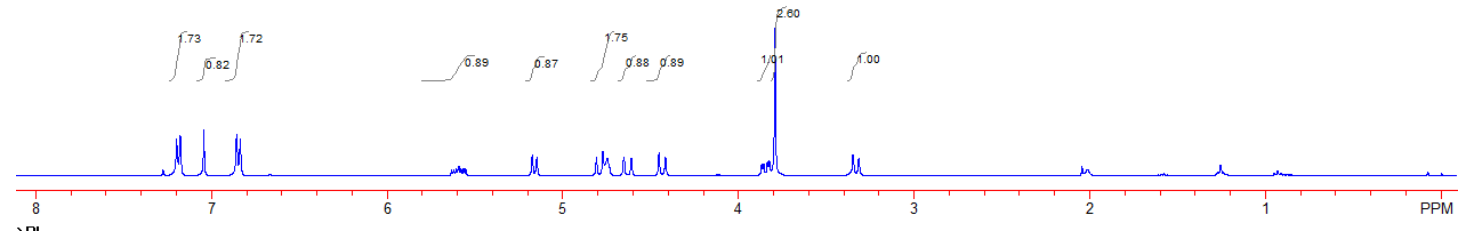




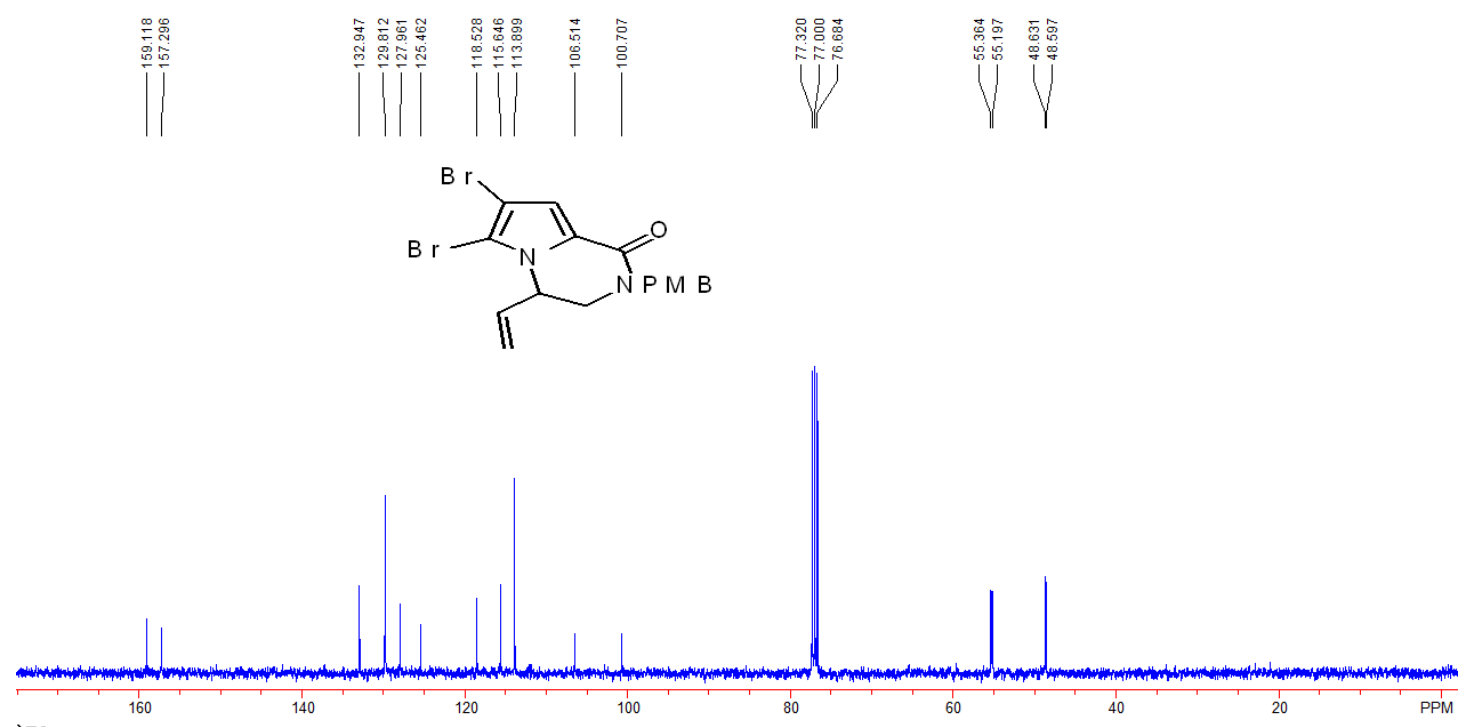

\section{NMR Spectra of $\mathbf{3 1}$}

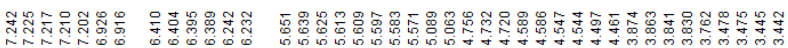

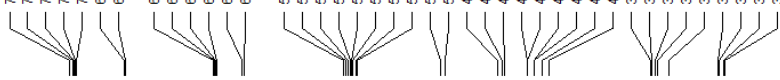

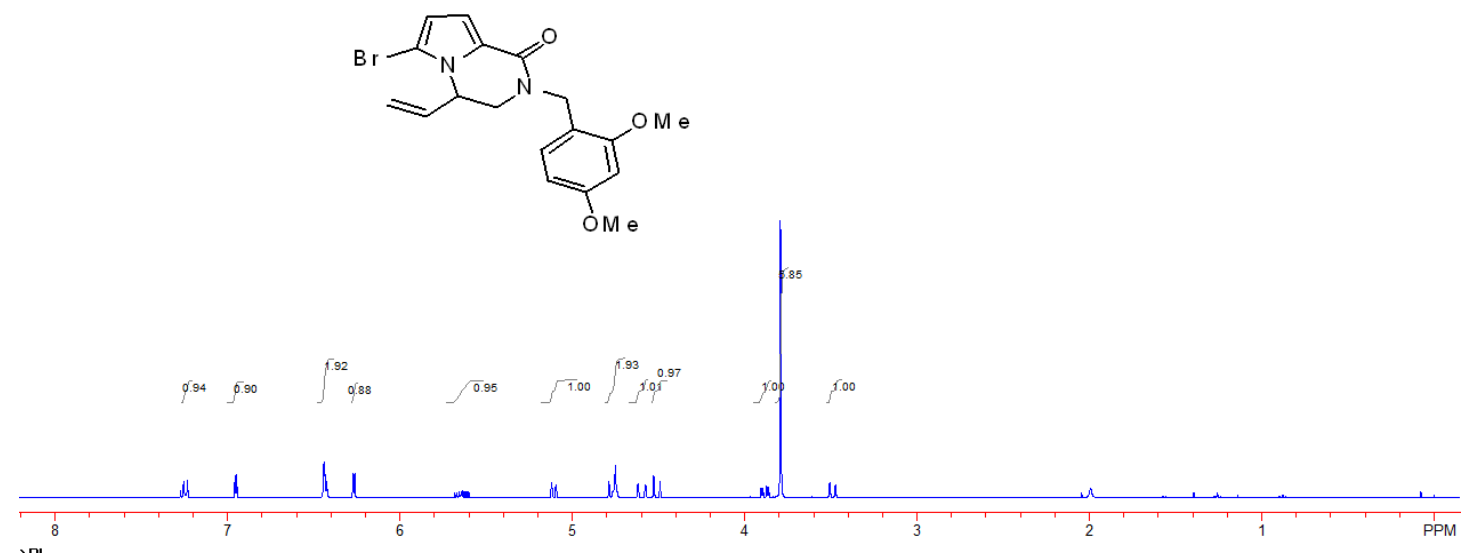




\section{Copies of HPLC Chromatographs}<smiles>C=CC1C[NH2+]Cc2ccc(C(=O)c3ccccc3)n21</smiles>

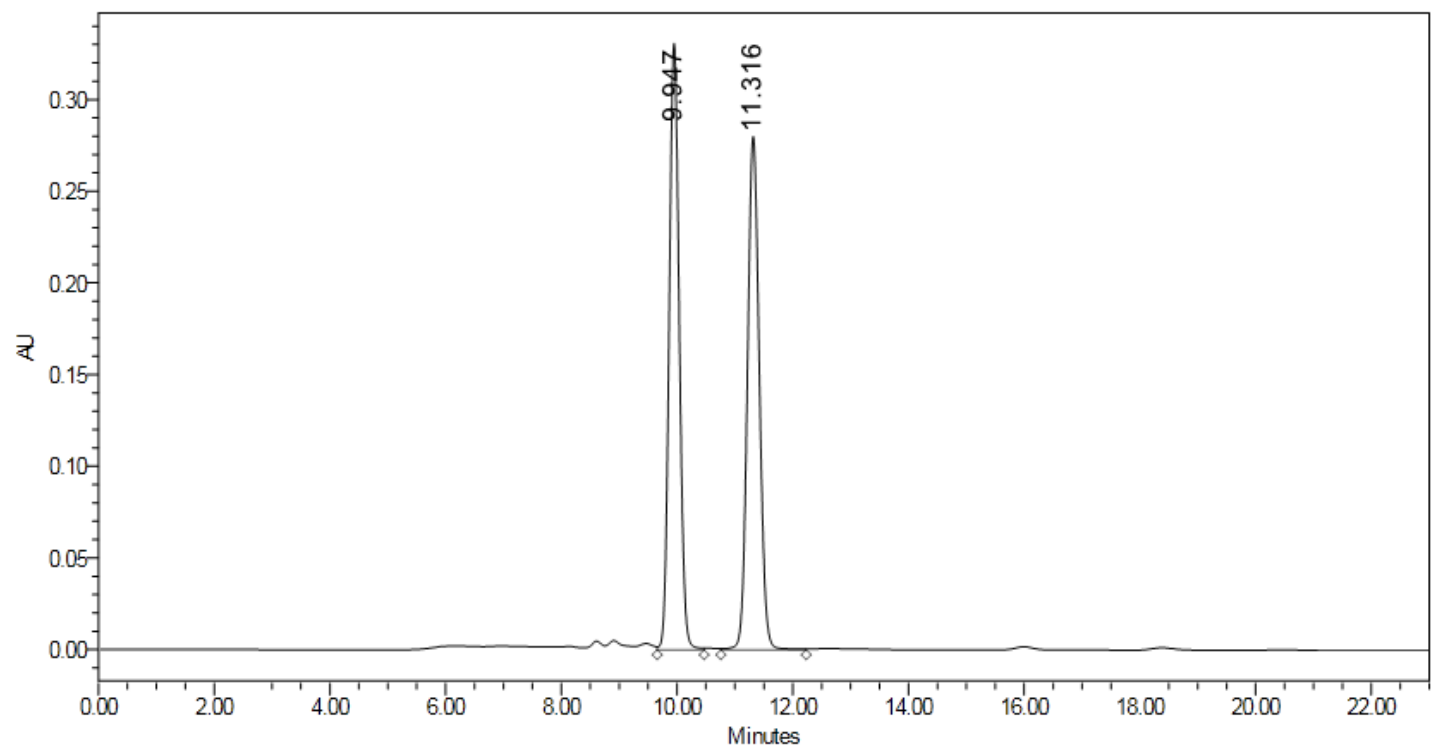

\begin{tabular}{|c|c|c|c|c|c|}
\hline & $\begin{array}{c}\text { RT } \\
(\mathrm{min})\end{array}$ & $\begin{array}{c}\text { Area } \\
\left(\mu \mathrm{V}^{*} \mathrm{sec}\right)\end{array}$ & $\%$ Area & $\begin{array}{c}\text { Height } \\
(\mu \mathrm{V})\end{array}$ & $\begin{array}{c}\% \\
\text { Height }\end{array}$ \\
\hline 1 & 9.947 & 3996441 & 50.07 & 330966 & 54.16 \\
\hline 2 & 11.316 & 3984640 & 49.93 & 280104 & 45.84 \\
\hline
\end{tabular}

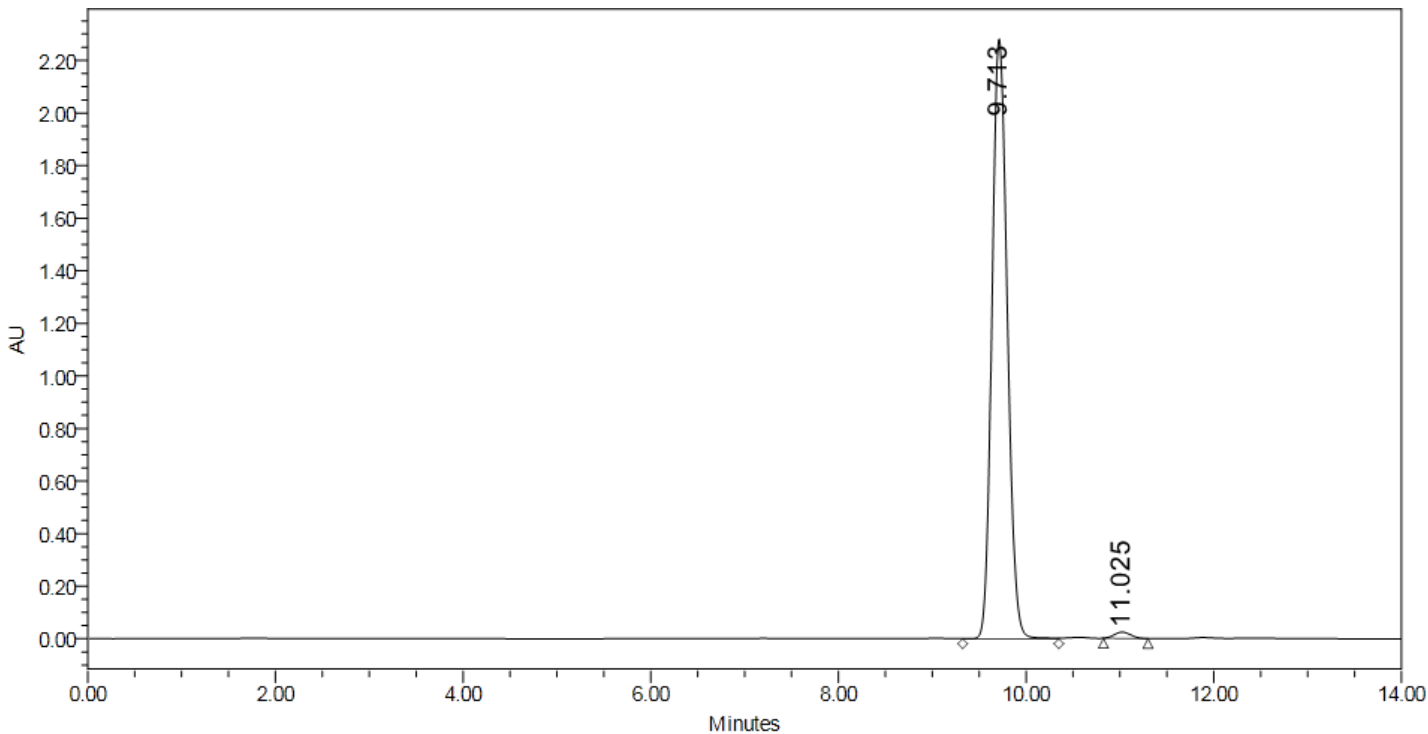

\begin{tabular}{|l|c|r|r|r|r|}
\hline & $\begin{array}{c}\text { RT } \\
(\mathrm{min})\end{array}$ & \multicolumn{1}{c|}{$\begin{array}{c}\text { Area } \\
\left(\mu \mathrm{V}^{*} \sec \right)\end{array}$} & $\%$ Area & $\begin{array}{c}\text { Height } \\
(\mu \mathrm{V})\end{array}$ & $\begin{array}{c}\% \\
\text { Height }\end{array}$ \\
\hline 1 & 9.713 & 25860995 & 98.88 & 2283331 & 98.96 \\
\hline 2 & 11.025 & 293212 & 1.12 & 24020 & 1.04 \\
\hline
\end{tabular}


<smiles>C=CCN1Cc2ccc(C(=O)c3ccccc3)n2C(C=C)C1</smiles>

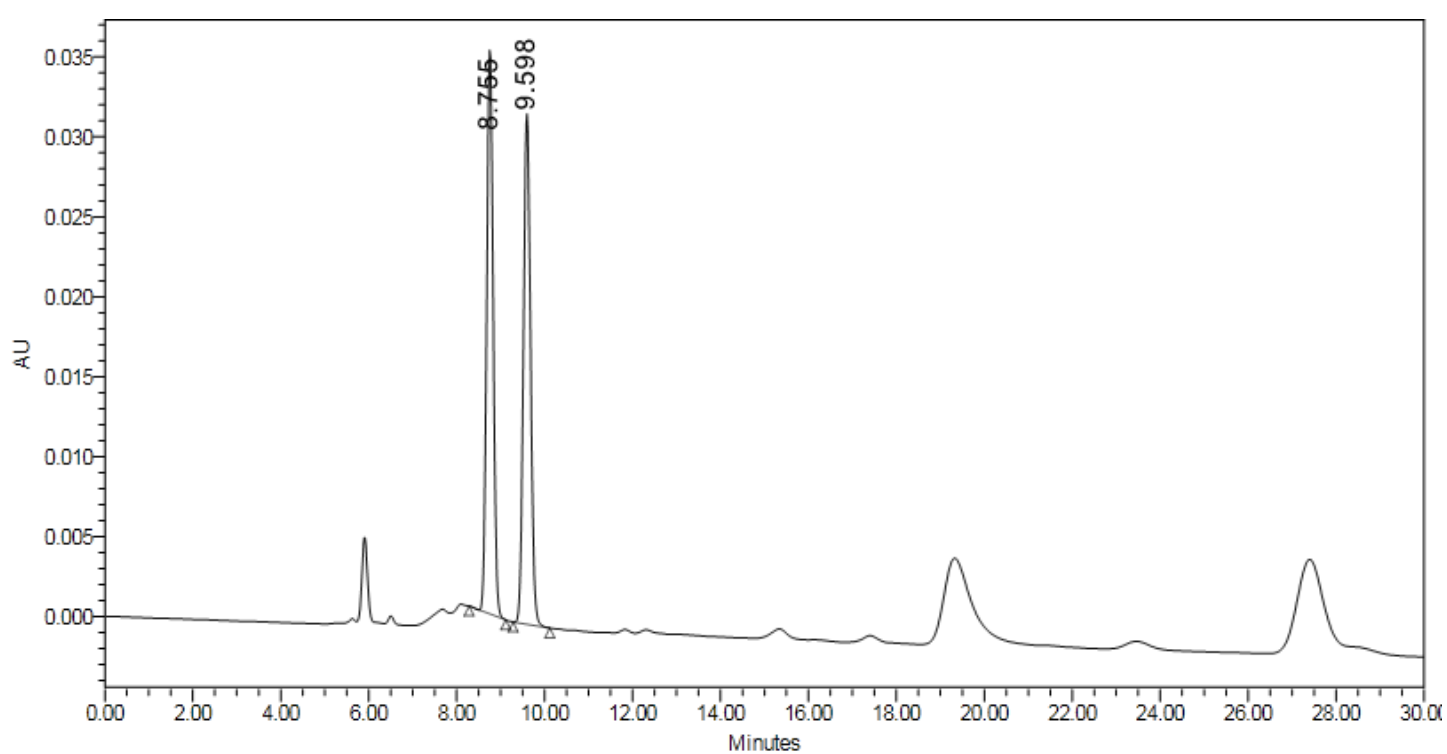

\begin{tabular}{|c|c|c|c|c|c|}
\hline & $\begin{array}{c}\mathrm{RT} \\
(\mathrm{min})\end{array}$ & $\begin{array}{c}\text { Area } \\
\left(\mu \mathrm{V}^{*} \mathrm{sec}\right)\end{array}$ & $\%$ Area & $\begin{array}{c}\text { Height } \\
(\mu \mathrm{V})\end{array}$ & $\begin{array}{c}\% \\
\text { Height }\end{array}$ \\
\hline 1 & 8.755 & 359419 & 50.06 & 35310 & 52.52 \\
\hline 2 & 9.598 & 358589 & 49.94 & 31920 & 47.48 \\
\hline
\end{tabular}

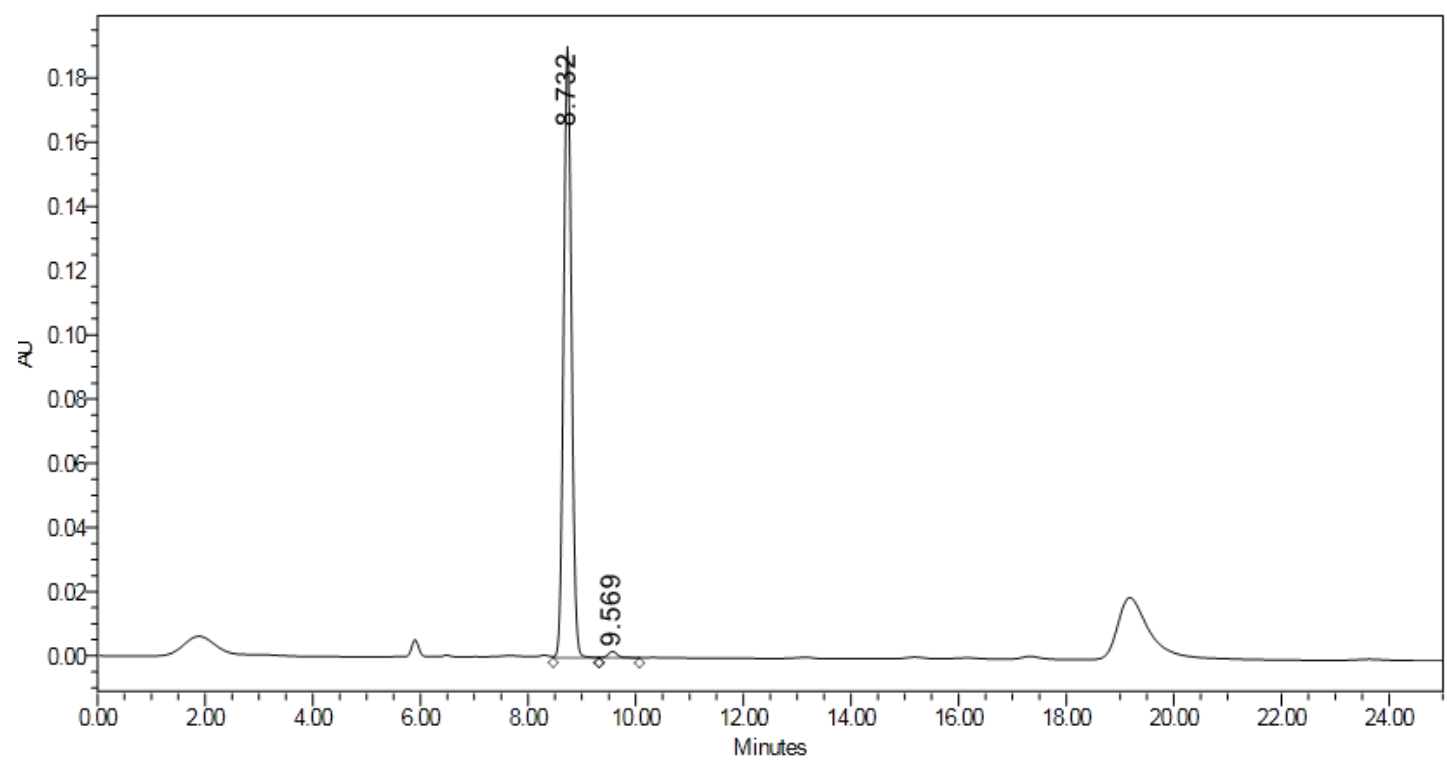

\begin{tabular}{|l|c|r|r|r|r|}
\hline & $\begin{array}{c}\mathrm{RT} \\
(\mathrm{min})\end{array}$ & $\begin{array}{c}\text { Area } \\
\left(\mu \mathrm{V}^{*} \mathrm{sec}\right)\end{array}$ & $\%$ Area & $\begin{array}{c}\text { Height } \\
(\mu \mathrm{V})\end{array}$ & $\begin{array}{c}\% \\
\text { Height }\end{array}$ \\
\hline 1 & 8.732 & 1939386 & 98.75 & 190200 & 98.99 \\
\hline 2 & 9.569 & 24464 & 1.25 & 1948 & 1.01 \\
\hline
\end{tabular}


<smiles>C=CC1CN(C(=O)OC(C)(C)C)Cc2ccc(C(=O)c3ccccc3)n21</smiles>

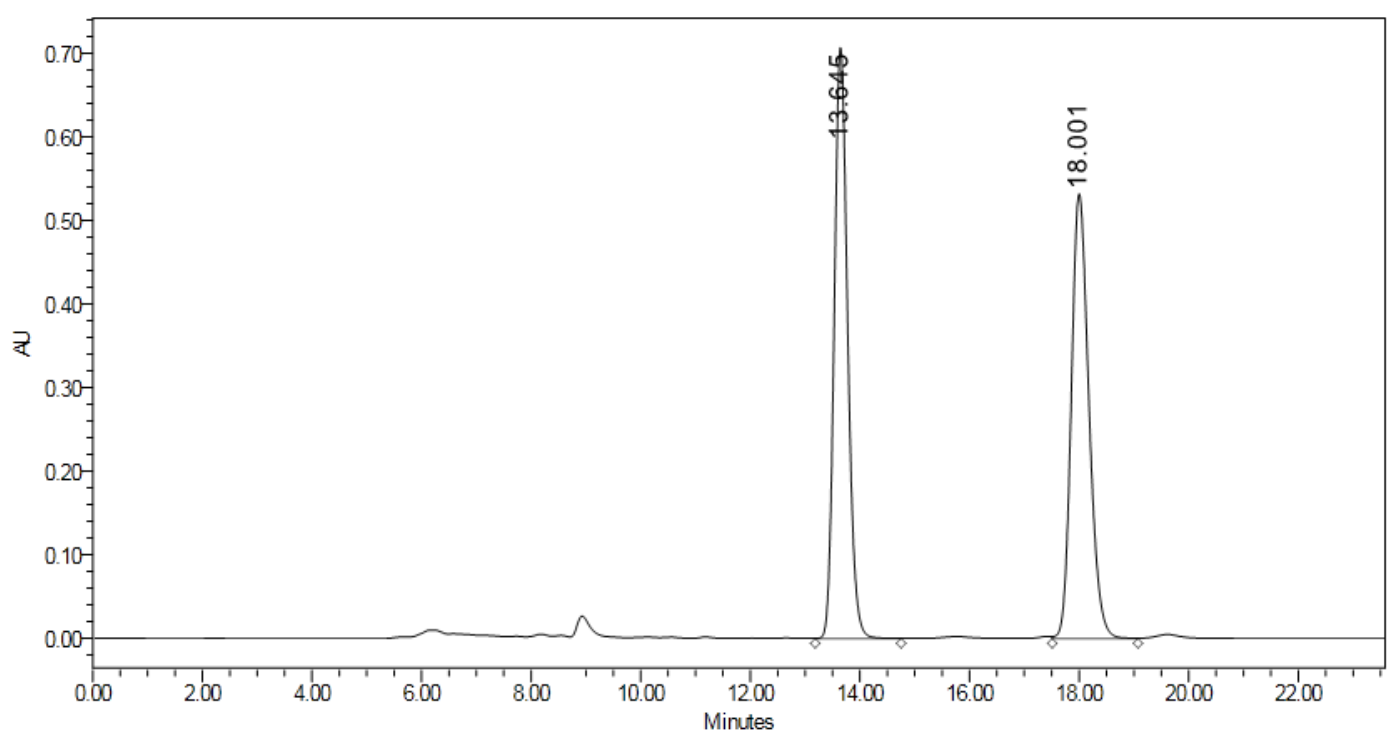

\begin{tabular}{|c|c|c|c|c|c|}
\hline & $\begin{array}{c}\mathrm{RT} \\
(\mathrm{min})\end{array}$ & $\begin{array}{c}\text { Area } \\
\left(\mu \mathrm{V}^{*} \mathrm{sec}\right)\end{array}$ & $\%$ Area & $\begin{array}{c}\text { Height } \\
(\mu \mathrm{V})\end{array}$ & $\begin{array}{c}\% \\
\text { Height }\end{array}$ \\
\hline 1 & 13.645 & 11973915 & 50.25 & 707152 & 57.06 \\
\hline 2 & 18.001 & 11852619 & 49.75 & 532175 & 42.94 \\
\hline
\end{tabular}

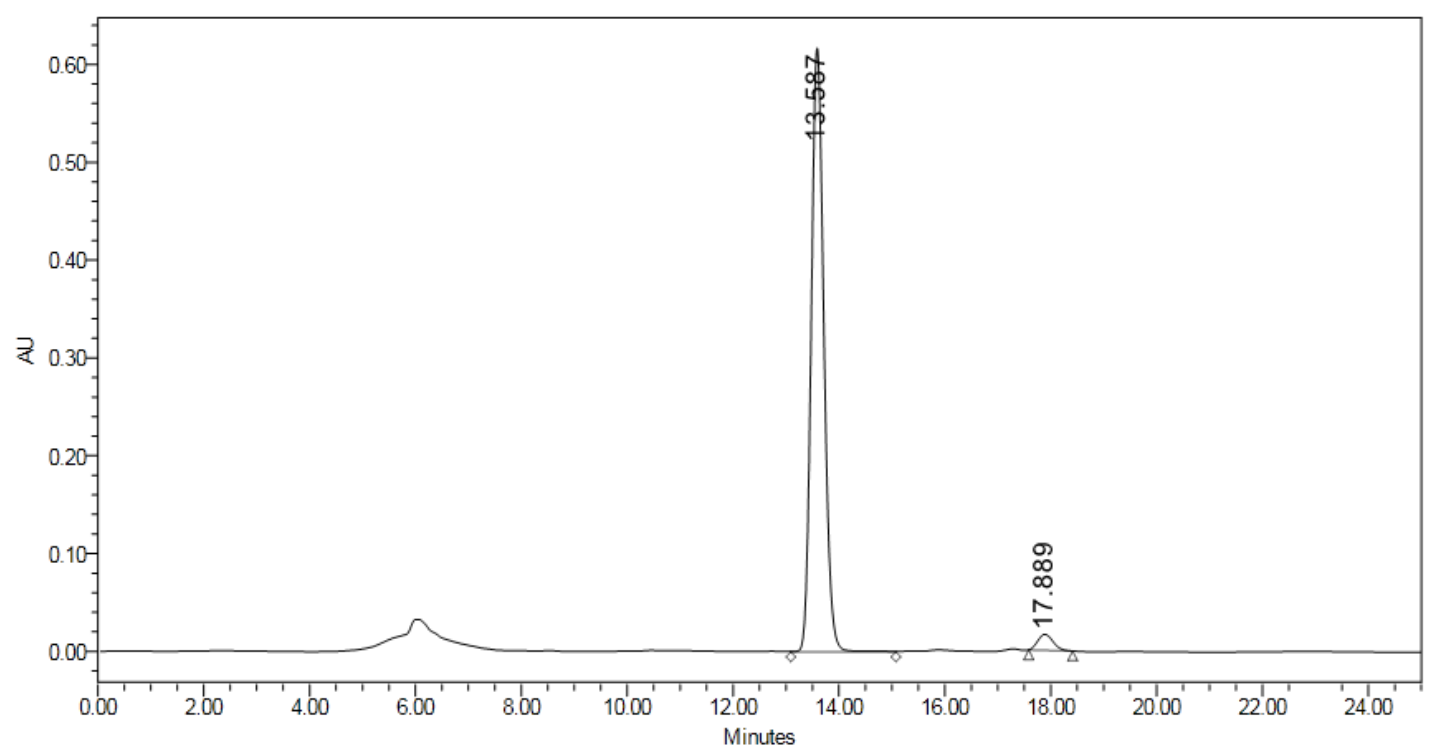

\begin{tabular}{|l|c|r|r|r|r|}
\hline & $\begin{array}{c}\mathrm{RT} \\
(\mathrm{min})\end{array}$ & $\begin{array}{c}\text { Area } \\
\left(\mu \mathrm{V}^{*} \mathrm{sec}\right)\end{array}$ & $\%$ Area & $\begin{array}{c}\text { Height } \\
(\mu \mathrm{V})\end{array}$ & $\begin{array}{c}\% \\
\text { Height }\end{array}$ \\
\hline 1 & 13.587 & 10240704 & 96.88 & 617672 & 97.41 \\
\hline 2 & 17.889 & 330142 & 3.12 & 16401 & 2.59 \\
\hline
\end{tabular}


<smiles>C=CC1CCCc2ccc(C(=O)c3ccccc3)n21</smiles>

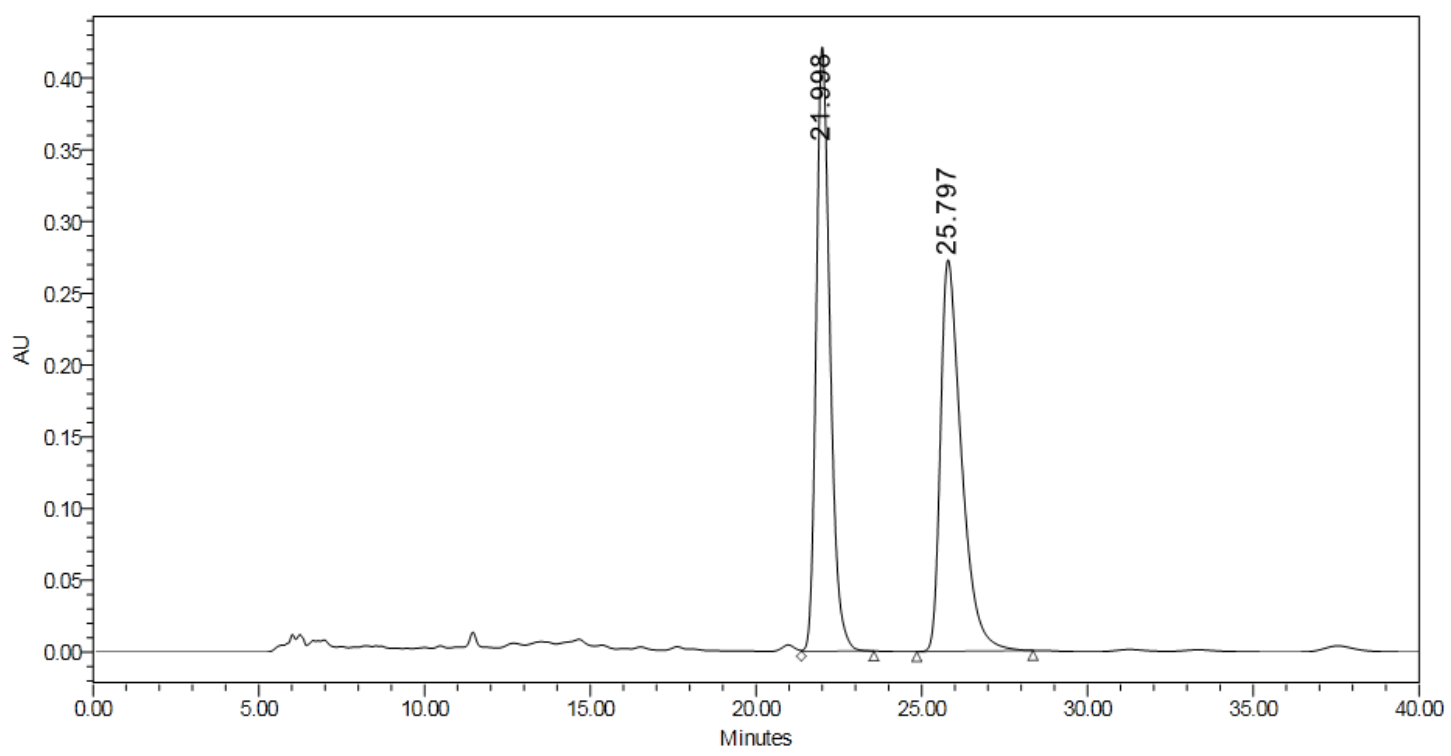

\begin{tabular}{|c|c|c|c|c|c|}
\hline & $\begin{array}{c}\mathrm{RT} \\
(\mathrm{min})\end{array}$ & $\begin{array}{c}\text { Area } \\
\left(\mu \mathrm{V}^{*} \mathrm{sec}\right)\end{array}$ & $\%$ Area & $\begin{array}{c}\text { Height } \\
(\mu \mathrm{V})\end{array}$ & $\begin{array}{c}\% \\
\text { Height }\end{array}$ \\
\hline 1 & 21.998 & 12196877 & 50.44 & $42111 \varepsilon$ & 60.68 \\
\hline 2 & 25.797 & 11984417 & 49.56 & 272826 & 39.32 \\
\hline
\end{tabular}

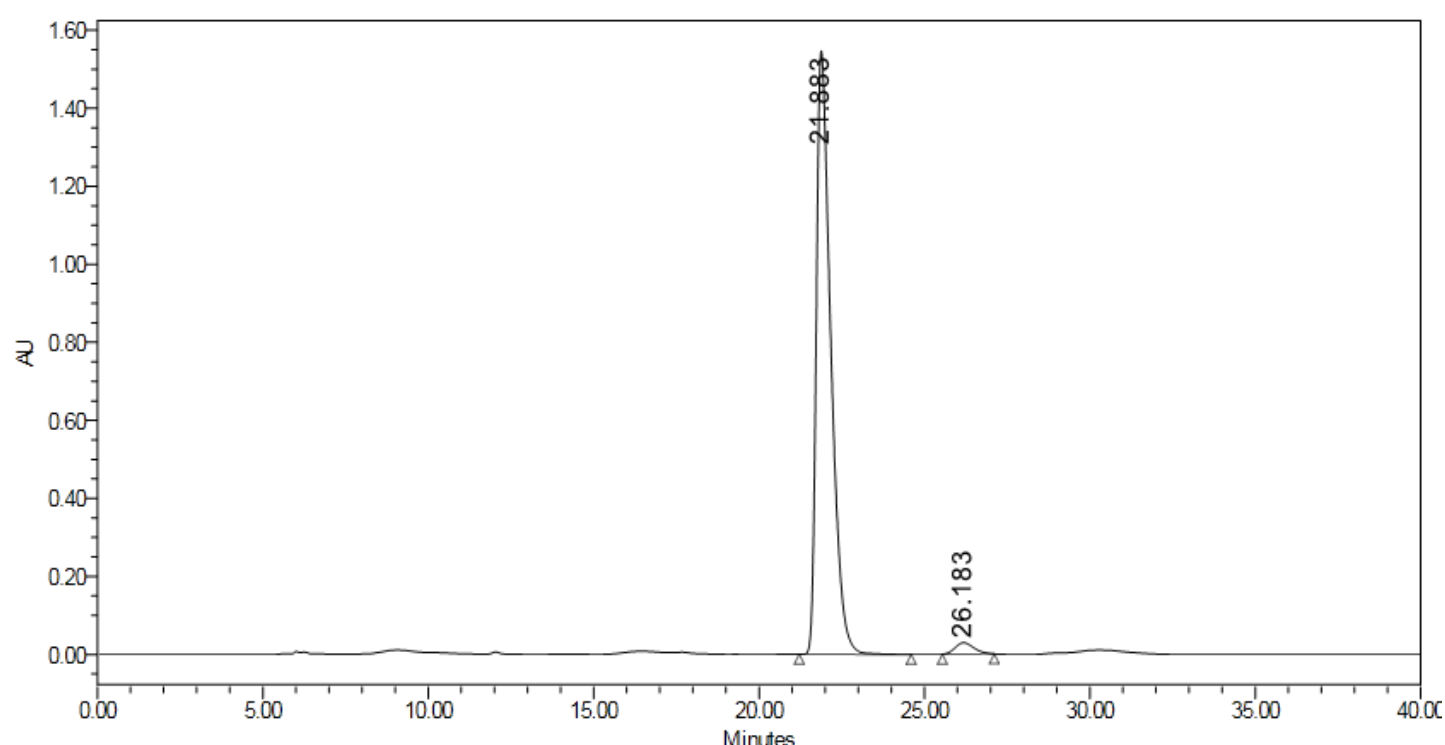

\begin{tabular}{|c|c|r|r|r|r|}
\hline & $\begin{array}{c}\mathrm{RT} \\
(\mathrm{min})\end{array}$ & $\begin{array}{c}\text { Area } \\
\left(\mu \mathrm{V}^{*} \mathrm{sec}\right)\end{array}$ & $\%$ Area & $\begin{array}{c}\text { Height } \\
(\mu \mathrm{V})\end{array}$ & $\begin{array}{c}\% \\
\text { Height }\end{array}$ \\
\hline 1 & 21.883 & 47032779 & 97.61 & 1546552 & 98.14 \\
\hline 2 & 26.183 & 1150523 & 2.39 & 29245 & 1.86 \\
\hline
\end{tabular}


<smiles>C=CC1C[NH2+]Cc2ccc(C(C)=O)n21</smiles>

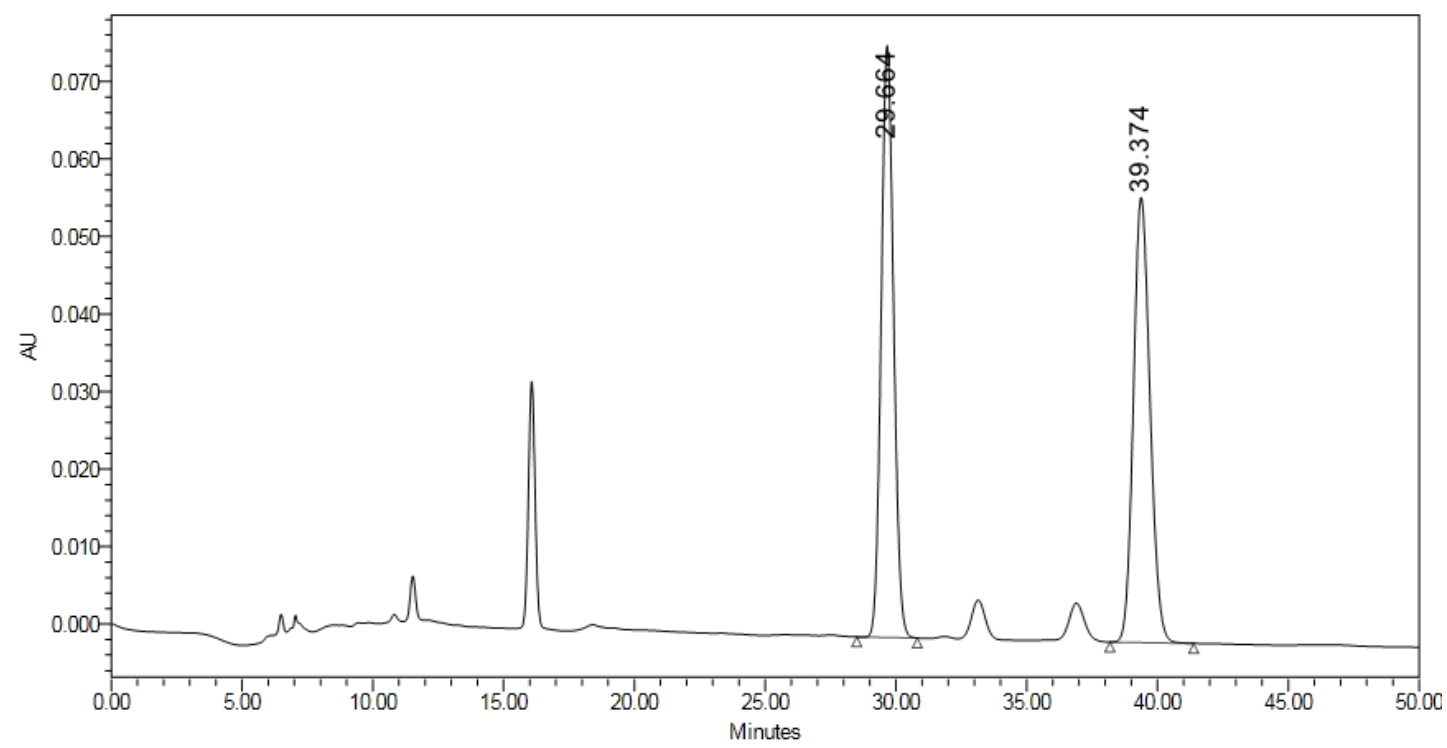

\begin{tabular}{|c|c|c|c|c|c|}
\hline & $\begin{array}{c}\mathrm{RT} \\
(\mathrm{min})\end{array}$ & $\begin{array}{c}\text { Area } \\
\left(\mu \mathrm{V}^{*} \text { sec }\right)\end{array}$ & $\%$ Area & $\begin{array}{c}\text { Height } \\
(\mu \mathrm{V})\end{array}$ & $\begin{array}{c}\% \\
\text { Height }\end{array}$ \\
\hline 1 & 29.664 & 2518240 & 49.85 & 76403 & 57.09 \\
\hline 2 & 39.374 & 2533703 & 50.15 & 57416 & 42.91 \\
\hline
\end{tabular}

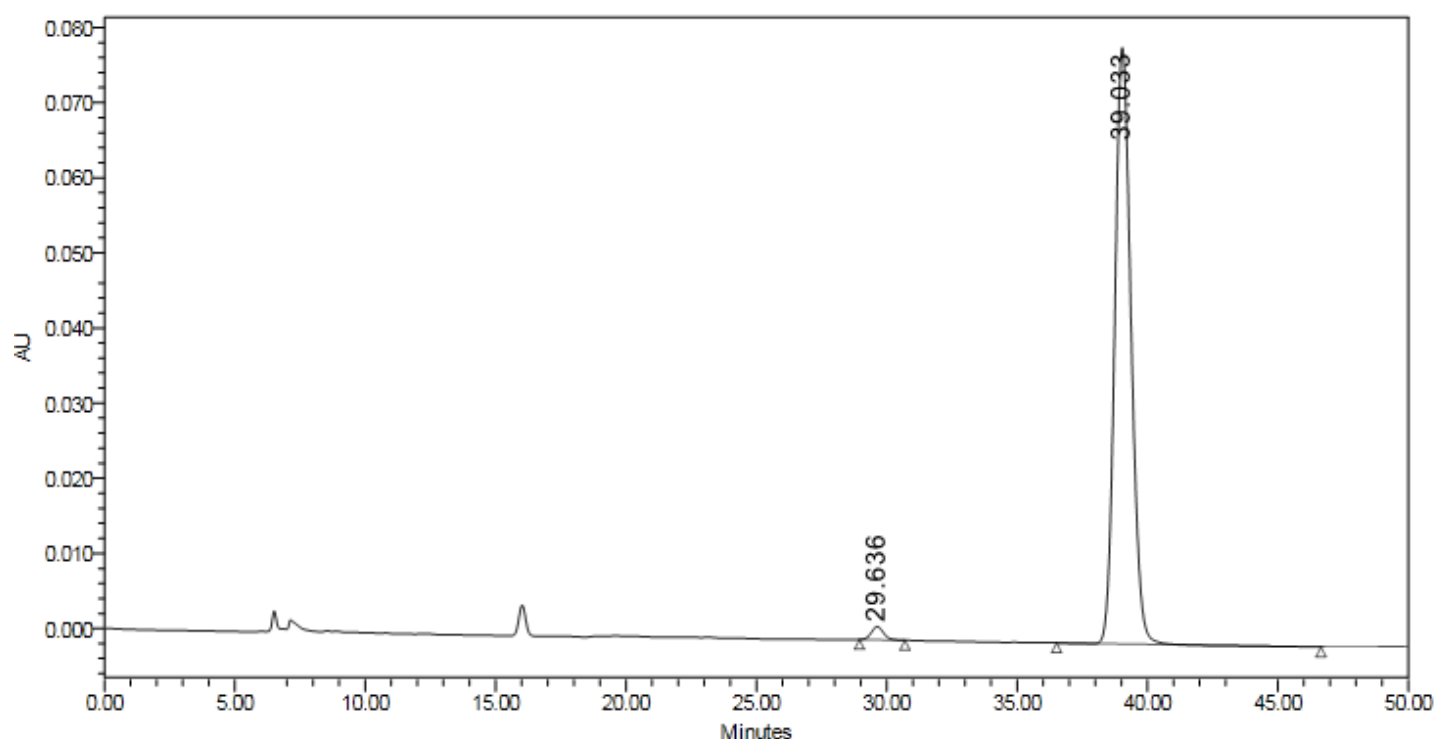

\begin{tabular}{|l|c|r|r|r|r|}
\hline & $\begin{array}{c}\mathrm{RT} \\
(\mathrm{min})\end{array}$ & $\begin{array}{c}\text { Area } \\
\left(\mu \mathrm{V}^{*} \mathrm{sec}\right)\end{array}$ & $\%$ Area & $\begin{array}{c}\text { Height } \\
(\mu \mathrm{V})\end{array}$ & $\begin{array}{c}\% \\
\text { Height }\end{array}$ \\
\hline 1 & 29.636 & 56869 & 1.63 & 1746 & 2.15 \\
\hline 2 & 39.033 & 3441549 & 98.37 & 79398 & 97.85 \\
\hline
\end{tabular}



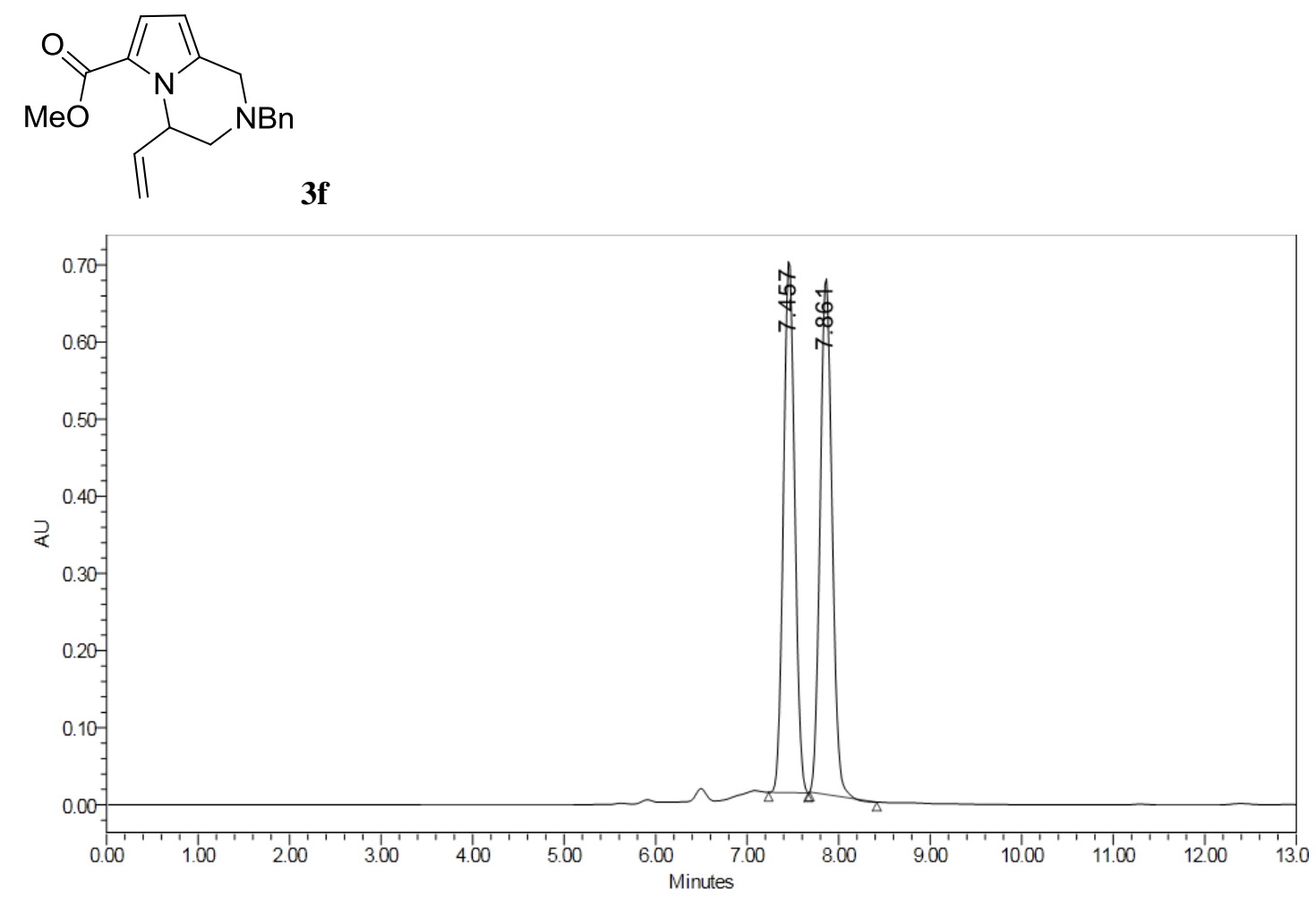

\begin{tabular}{|c|c|c|c|c|c|}
\hline & $\begin{array}{c}\mathrm{RT} \\
(\mathrm{min})\end{array}$ & $\begin{array}{c}\text { Area } \\
\left(\mu \mathrm{V}^{*} \mathrm{sec}\right)\end{array}$ & $\%$ Area & $\begin{array}{c}\text { Height } \\
(\mu \mathrm{V})\end{array}$ & $\begin{array}{c}\% \\
\text { Height }\end{array}$ \\
\hline 1 & 7.457 & 5908040 & 49.28 & 691916 & 50.80 \\
\hline 2 & 7.861 & 6080166 & 50.72 & 670246 & 49.20 \\
\hline
\end{tabular}

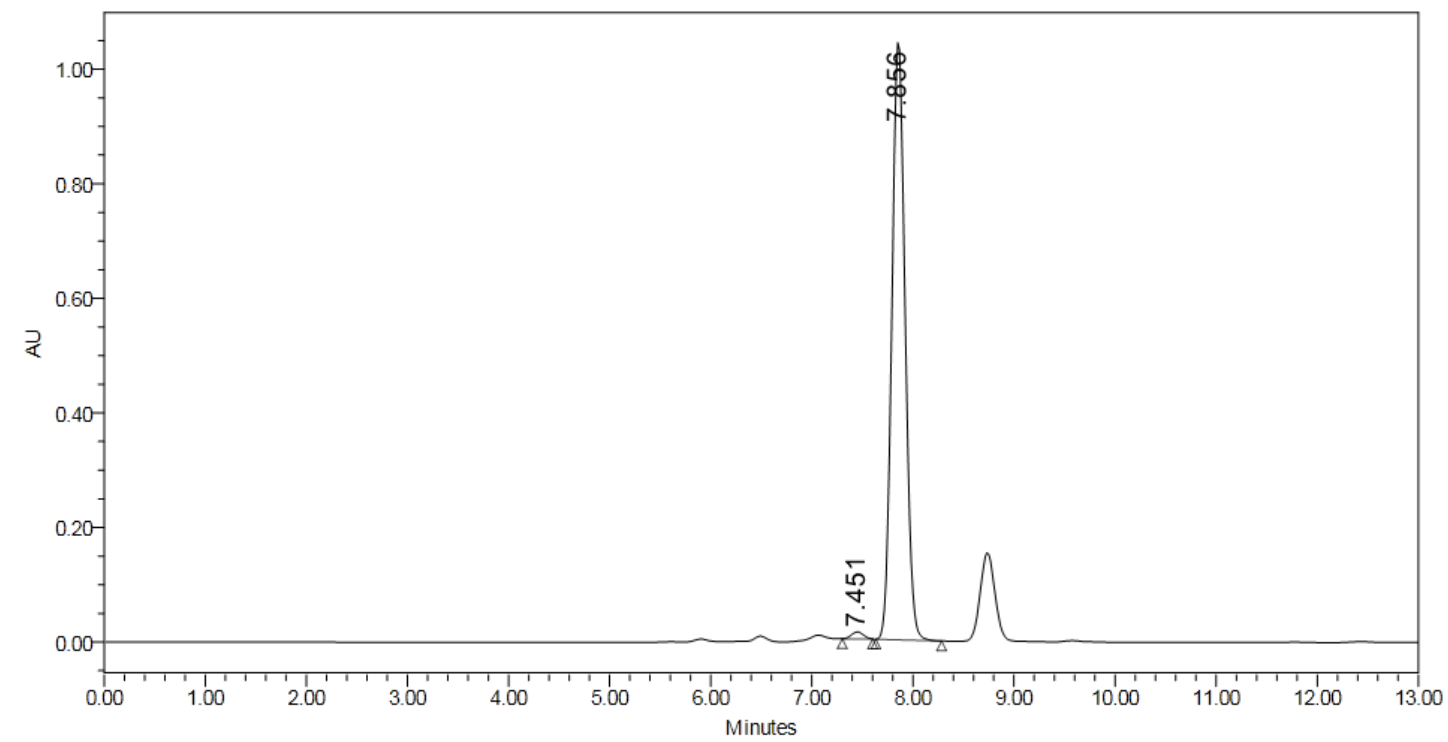

\begin{tabular}{|l|c|r|r|r|r|}
\hline & $\begin{array}{c}\mathrm{RT} \\
(\mathrm{min})\end{array}$ & $\begin{array}{c}\text { Area } \\
\left(\mu \mathrm{V}^{*} \mathrm{sec}\right)\end{array}$ & $\%$ Area & $\begin{array}{c}\text { Height } \\
(\mu \mathrm{V})\end{array}$ & $\begin{array}{c}\% \\
\text { Height }\end{array}$ \\
\hline 1 & 7.451 & 99591 & 1.04 & 12195 & 1.15 \\
\hline 2 & 7.856 & 9472656 & 98.96 & 1045085 & 98.85 \\
\hline
\end{tabular}


<smiles>C=CC1CN(Cc2ccccc2)Cc2ccc(C(=O)OC(C)(C)C)n21</smiles>
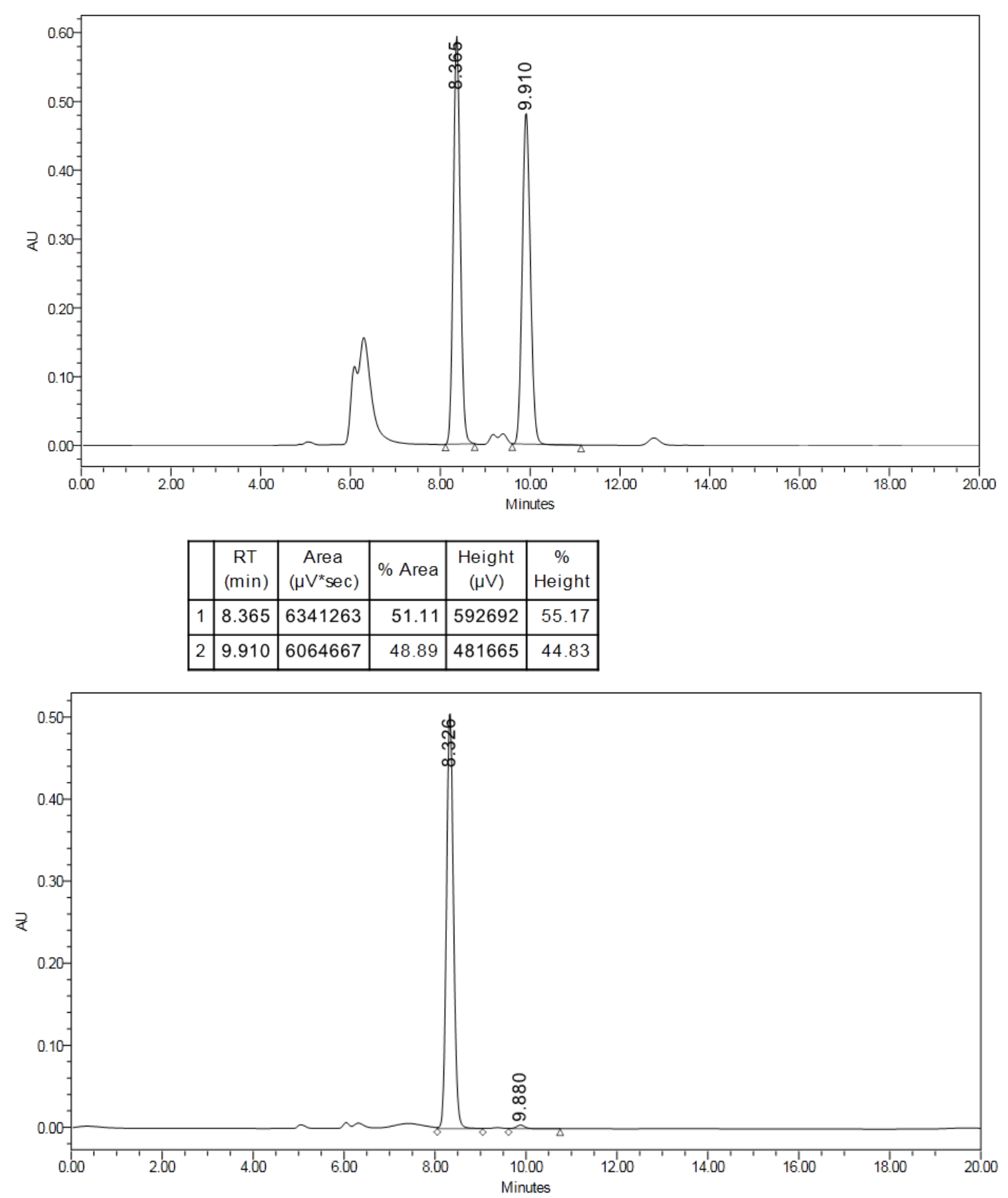

\begin{tabular}{|l|c|r|r|r|r|}
\hline & $\begin{array}{c}\mathrm{RT} \\
(\mathrm{min})\end{array}$ & $\begin{array}{c}\text { Area } \\
\left(\mu \mathrm{V}^{*} \mathrm{sec}\right)\end{array}$ & $\%$ Area & $\begin{array}{c}\text { Height } \\
(\mu \mathrm{V})\end{array}$ & $\begin{array}{c}\% \\
\text { Height }\end{array}$ \\
\hline 1 & 8.326 & 5445583 & 98.88 & 507970 & 99.13 \\
\hline 2 & 9.880 & 61706 & 1.12 & 4465 & 0.87 \\
\hline
\end{tabular}


<smiles>C=CC1C[NH2+]Cc2ccc(C(=O)O)n2C1c1ccccc1</smiles>

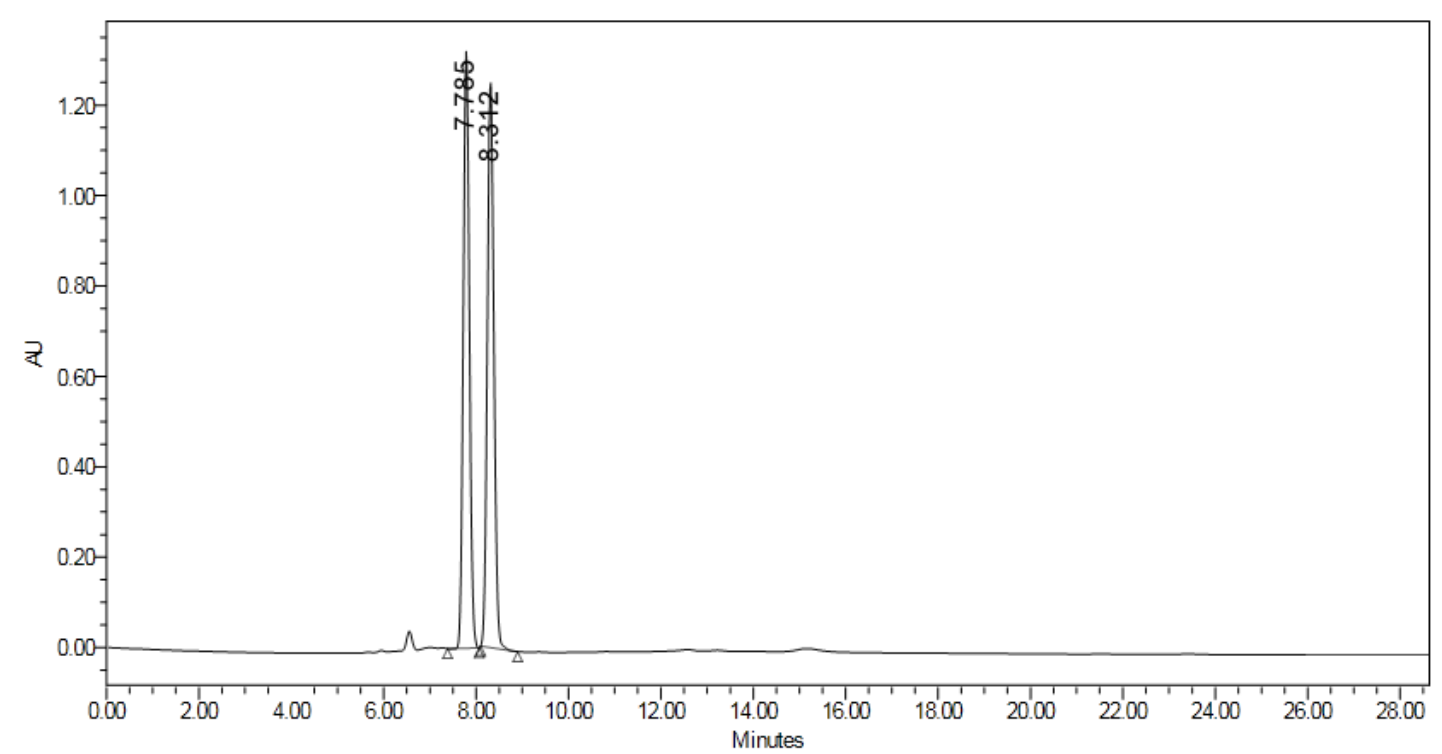

\begin{tabular}{|c|c|c|c|c|c|}
\hline & $\begin{array}{c}\mathrm{RT} \\
(\mathrm{min})\end{array}$ & $\begin{array}{c}\text { Area } \\
\left(\mu \mathrm{V}^{*} \mathrm{sec}\right)\end{array}$ & $\%$ Area & $\begin{array}{c}\text { Height } \\
(\mu \mathrm{V})\end{array}$ & $\begin{array}{c}\% \\
\text { Height }\end{array}$ \\
\hline 1 & 7.785 & 12181895 & 49.72 & 1318372 & 51.31 \\
\hline 2 & 8.312 & 12319252 & 50.28 & 1251197 & 48.69 \\
\hline
\end{tabular}

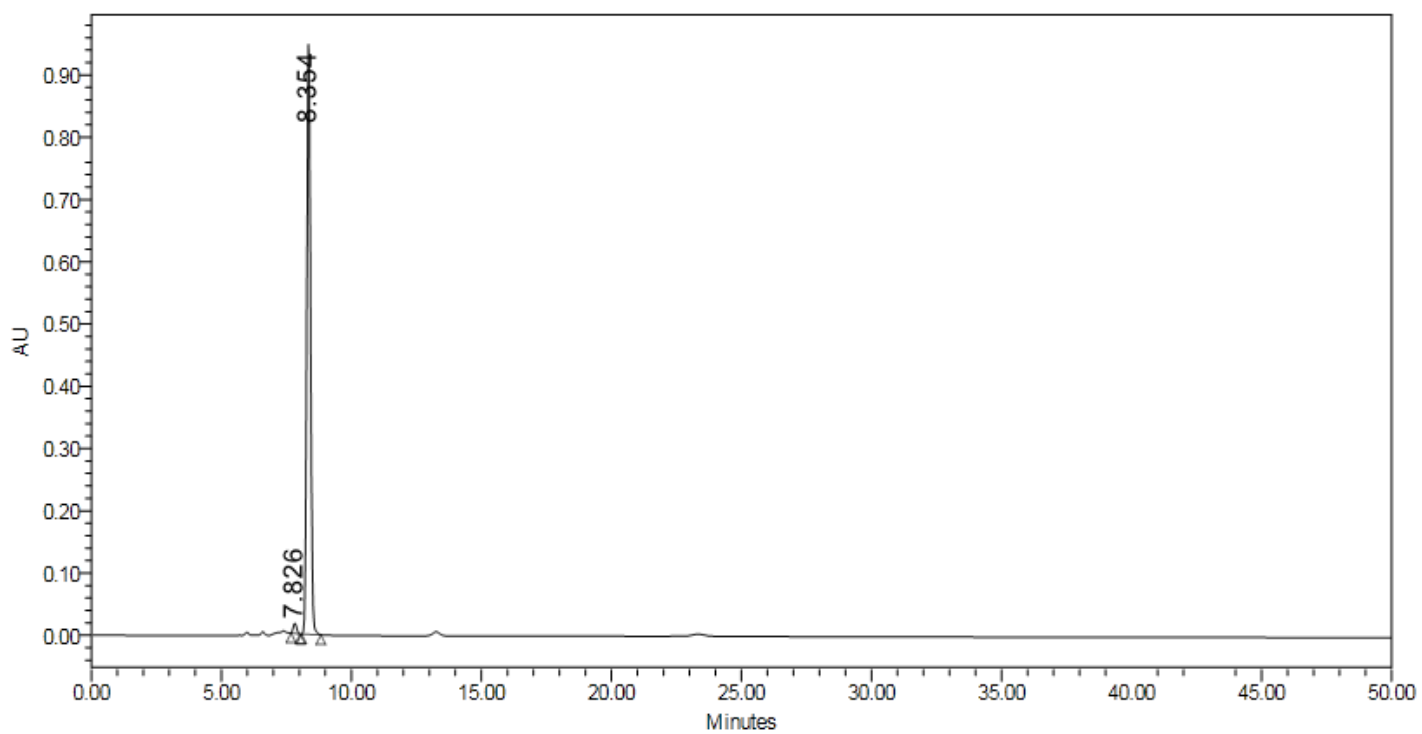

\begin{tabular}{|c|c|r|r|r|r|}
\hline & $\begin{array}{c}\mathrm{RT} \\
(\mathrm{min})\end{array}$ & $\begin{array}{c}\text { Area } \\
\left(\mu \mathrm{V}^{*} \text { sec }\right)\end{array}$ & $\%$ Area & $\begin{array}{c}\text { Height } \\
(\mu \mathrm{V})\end{array}$ & $\begin{array}{c}\% \\
\text { Height }\end{array}$ \\
\hline 1 & 7.826 & 149531 & 1.54 & 16631 & 1.72 \\
\hline 2 & 8.354 & 9563393 & 98.46 & 947989 & 98.28 \\
\hline
\end{tabular}


<smiles>C=CC1C[NH2+]C(Cc2ccccc2)C(=O)c2cccn21</smiles>

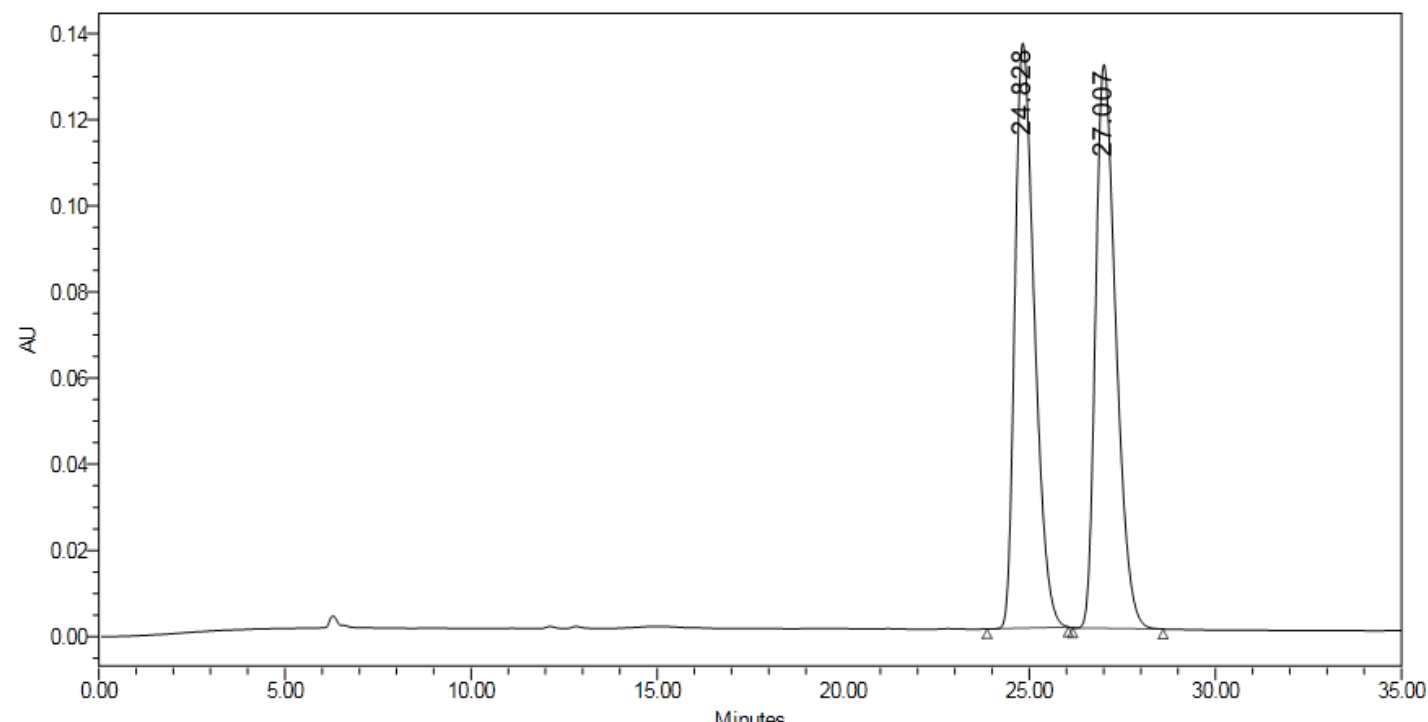

\begin{tabular}{|c|c|c|c|c|c|}
\hline & $\begin{array}{c}\mathrm{RT} \\
(\mathrm{min})\end{array}$ & $\begin{array}{c}\text { Area } \\
\left(\mu \mathrm{V}^{*} \mathrm{sec}\right)\end{array}$ & $\%$ Area & $\begin{array}{c}\text { Height } \\
(\mu \mathrm{V})\end{array}$ & $\begin{array}{c}\% \\
\text { Height }\end{array}$ \\
\hline 1 & 24.828 & 4939941 & 49.85 & 135887 & 50.92 \\
\hline 2 & 27.007 & 4970157 & 50.15 & 130967 & 49.08 \\
\hline
\end{tabular}

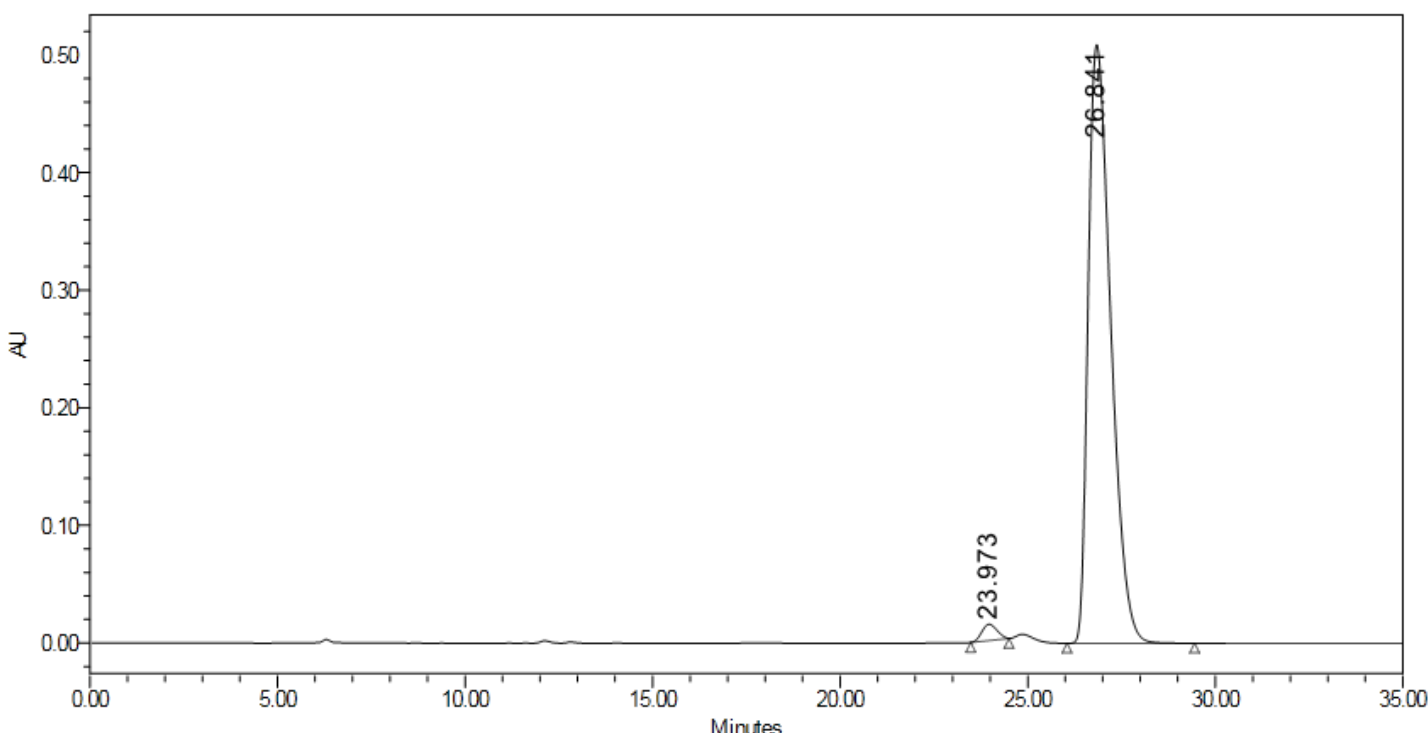

\begin{tabular}{|l|c|r|r|r|r|}
\hline & $\begin{array}{c}\text { RT } \\
(\mathrm{min})\end{array}$ & $\begin{array}{c}\text { Area } \\
\left(\mu \mathrm{V}^{*} \mathrm{sec}\right)\end{array}$ & $\%$ Area & $\begin{array}{c}\text { Height } \\
(\mu \mathrm{V})\end{array}$ & $\begin{array}{c}\% \\
\text { Height }\end{array}$ \\
\hline 1 & 23.973 & 389718 & 1.80 & 13810 & 2.64 \\
\hline 2 & 26.841 & 21258895 & 98.20 & 509081 & 97.36 \\
\hline
\end{tabular}


<smiles>C=CC1C[NH2+]C(=O)c2cc(Br)c(Br)n21</smiles>

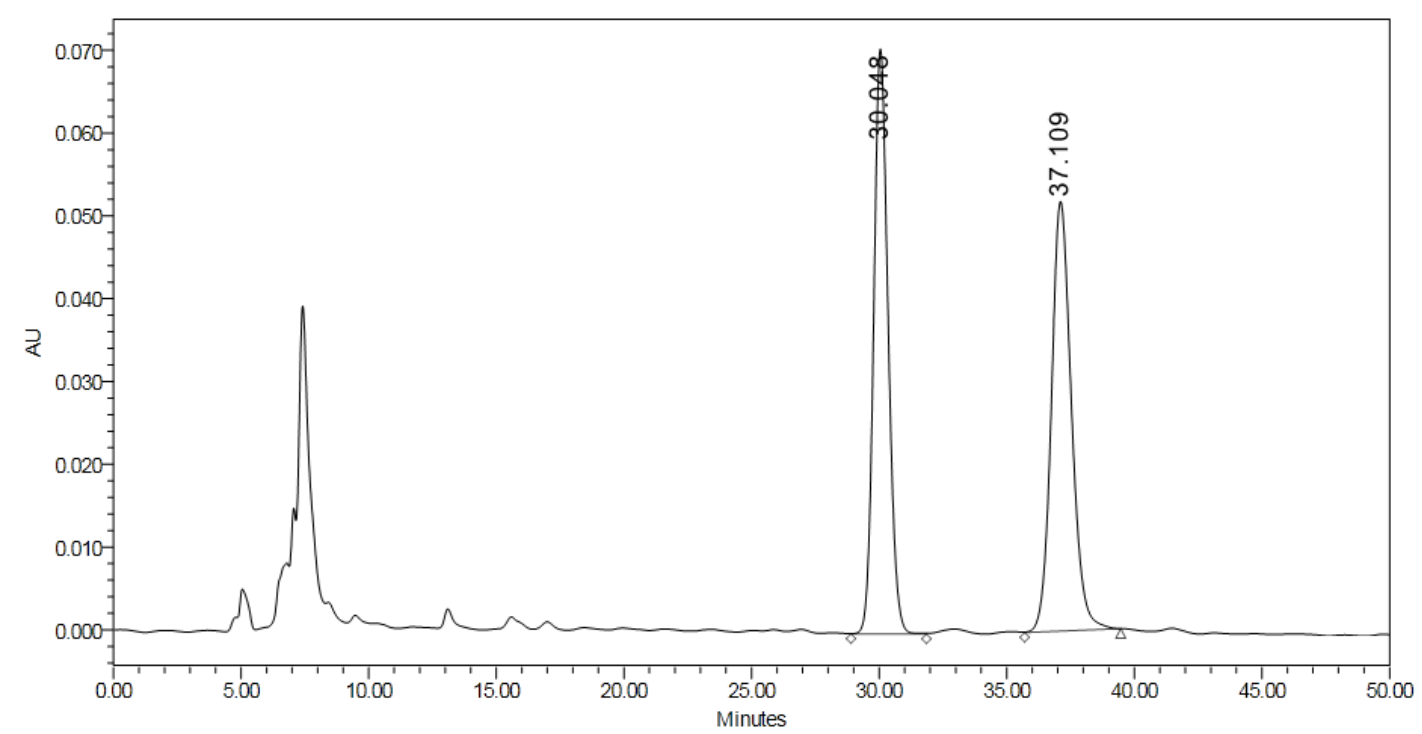

\begin{tabular}{|c|c|c|c|c|c|}
\hline & $\begin{array}{c}\mathrm{RT} \\
(\mathrm{min})\end{array}$ & $\begin{array}{c}\text { Area } \\
\left(\mu \mathrm{V}^{\star} \mathrm{sec}\right)\end{array}$ & $\%$ Area & $\begin{array}{c}\text { Height } \\
(\mu \mathrm{V})\end{array}$ & $\begin{array}{c}\% \\
\text { Height }\end{array}$ \\
\hline 1 & 30.048 & 2776108 & 49.47 & 70670 & 57.65 \\
\hline 2 & 37.109 & 2835091 & 50.53 & 51906 & 42.35 \\
\hline
\end{tabular}

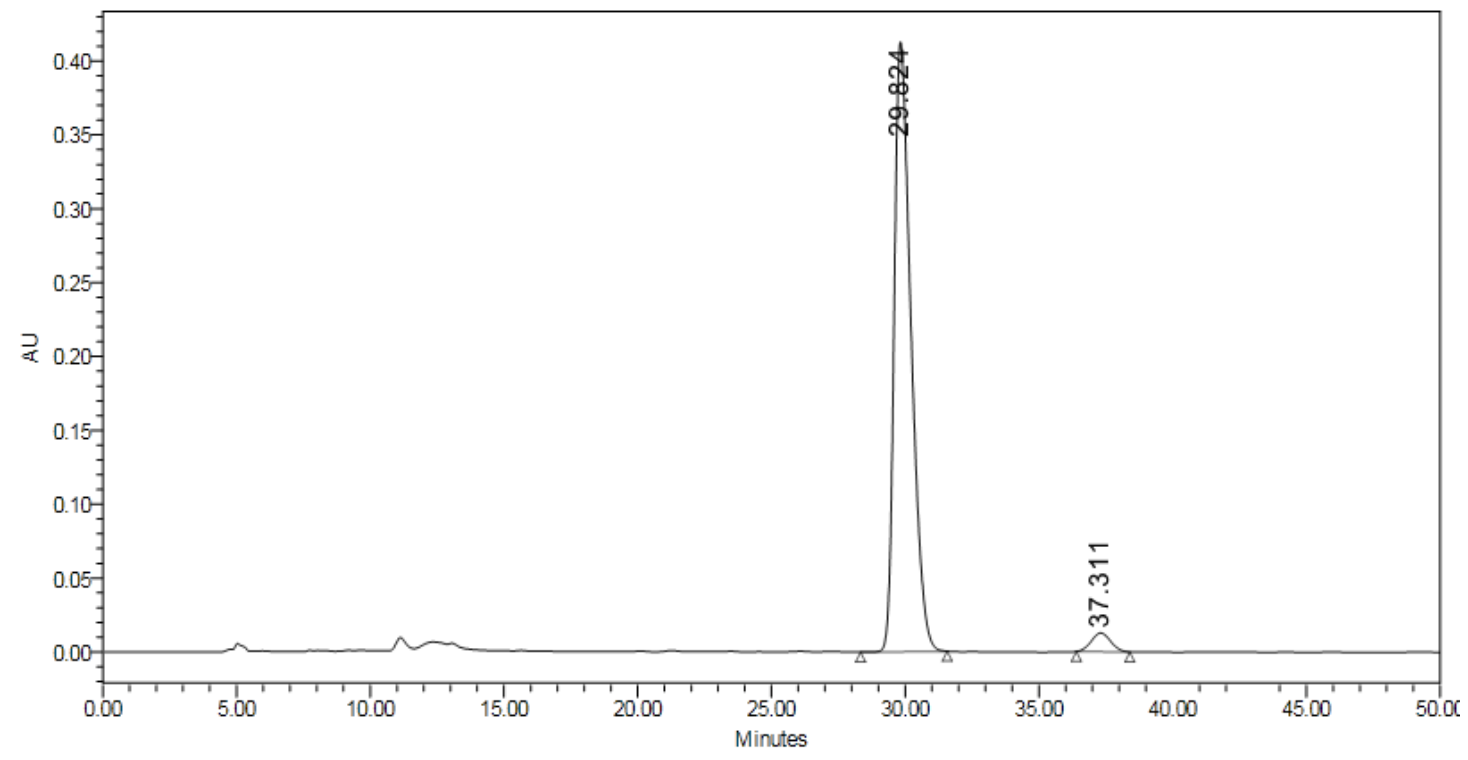

\begin{tabular}{|l|c|c|r|r|r|}
\hline & $\begin{array}{c}\mathrm{RT} \\
(\mathrm{min})\end{array}$ & $\begin{array}{c}\text { Area } \\
\left(\mu \mathrm{V}^{*} \sec \right)\end{array}$ & \% Area & $\begin{array}{c}\text { Height } \\
(\mu \mathrm{V})\end{array}$ & $\begin{array}{c}\% \\
\text { Height }\end{array}$ \\
\hline 1 & 29.824 & 18009084 & 96.59 & 412940 & 97.03 \\
\hline 2 & 37.311 & 636427 & 3.41 & 12652 & 2.97 \\
\hline
\end{tabular}


<smiles></smiles>

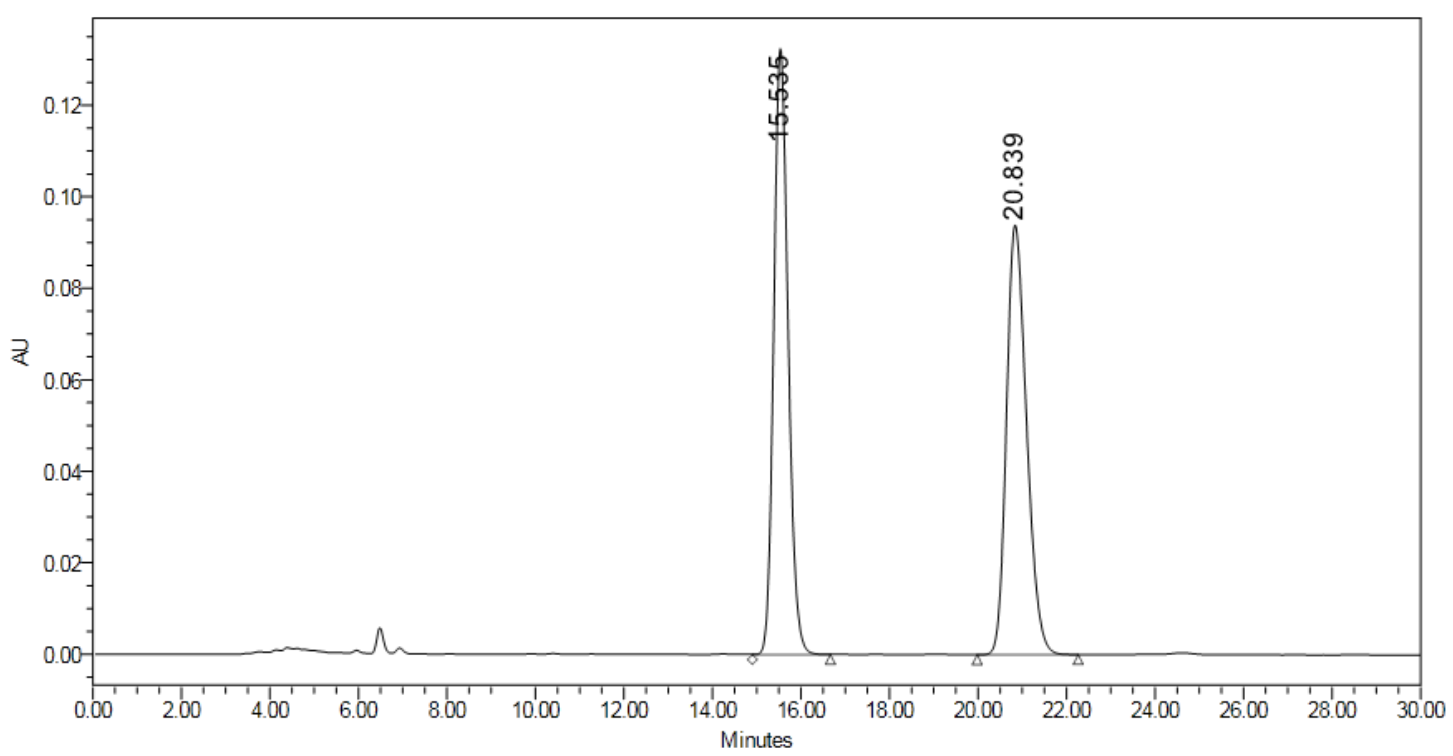

\begin{tabular}{|c|c|c|c|c|c|}
\hline & $\begin{array}{c}\mathrm{RT} \\
(\mathrm{min})\end{array}$ & $\begin{array}{c}\text { Area } \\
\left(\mu \mathrm{V}^{*} \text { sec }\right)\end{array}$ & $\%$ Area & $\begin{array}{c}\text { Height } \\
(\mu \mathrm{V})\end{array}$ & $\begin{array}{c}\% \\
\text { Height }\end{array}$ \\
\hline 1 & 15.535 & 2978783 & 50.15 & 132451 & 58.51 \\
\hline 2 & 20.839 & 2960606 & 49.85 & 93926 & 41.49 \\
\hline
\end{tabular}

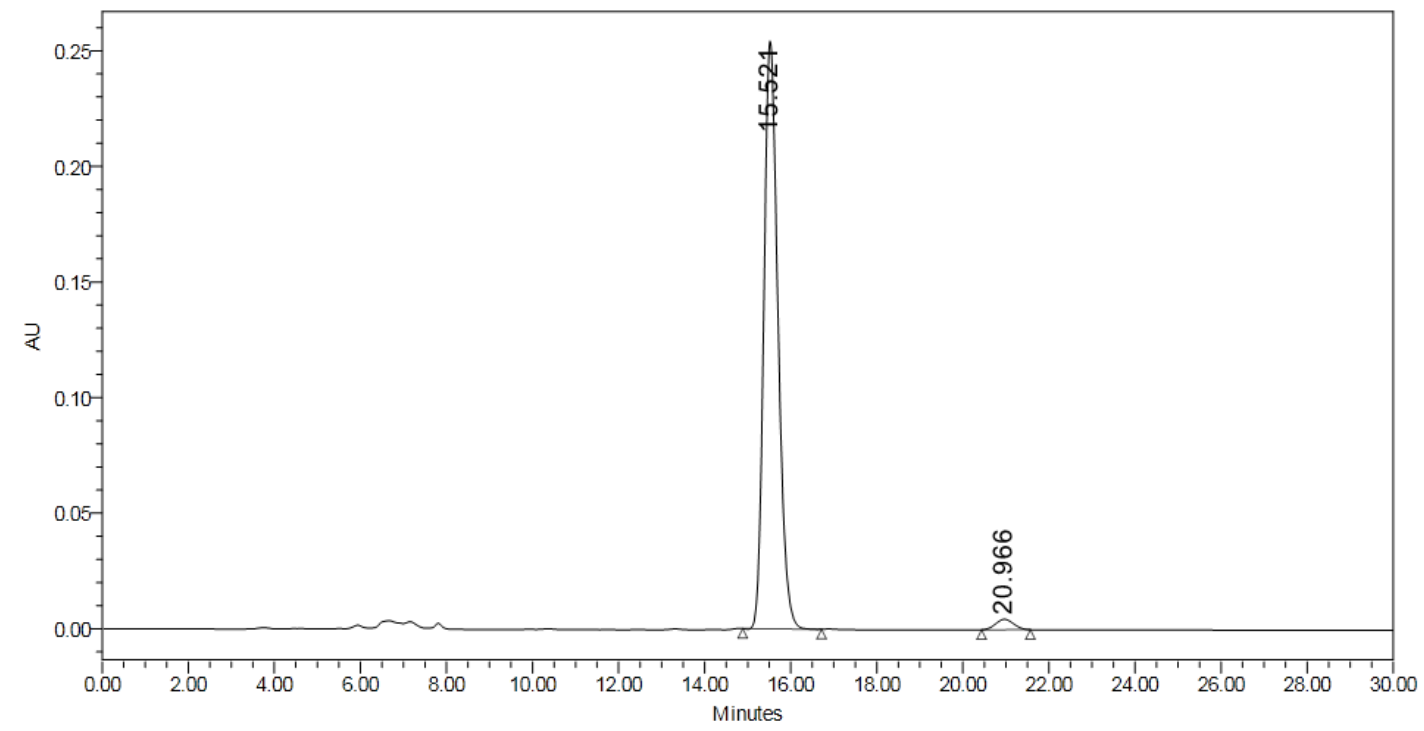

\begin{tabular}{|l|c|r|r|r|r|}
\hline & $\begin{array}{c}\mathrm{RT} \\
(\mathrm{min})\end{array}$ & $\begin{array}{c}\text { Area } \\
\left(\mu \mathrm{V}^{*} \mathrm{sec}\right)\end{array}$ & $\%$ Area & $\begin{array}{c}\text { Height } \\
(\mu \mathrm{V})\end{array}$ & $\begin{array}{c}\% \\
\text { Height }\end{array}$ \\
\hline 1 & 15.521 & 5775593 & 97.76 & 254368 & 98.25 \\
\hline 2 & 20.966 & 132341 & 2.24 & 4534 & 1.75 \\
\hline
\end{tabular}


<smiles>C=CC1CN(Cc2ccc(OC)cc2OC)C(=O)c2ccc(Br)n21</smiles>

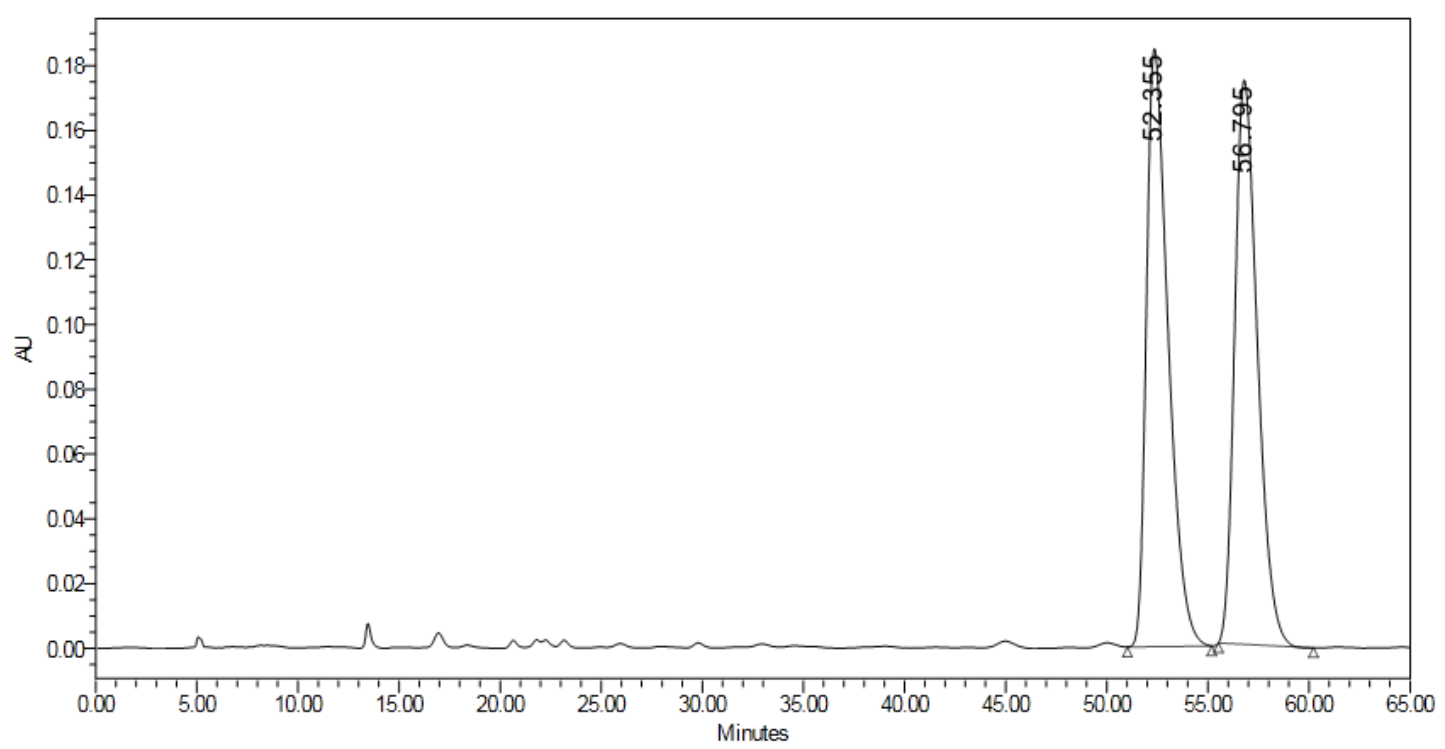

\begin{tabular}{|c|c|c|c|c|c|}
\hline & $\begin{array}{c}\mathrm{RT} \\
(\mathrm{min})\end{array}$ & $\begin{array}{c}\text { Area } \\
\left(\mu \mathrm{V}^{*} \mathrm{sec}\right)\end{array}$ & $\%$ Area & $\begin{array}{c}\text { Height } \\
(\mu \mathrm{V})\end{array}$ & $\begin{array}{c}\% \\
\text { Height }\end{array}$ \\
\hline 1 & 52.355 & 13784989 & 50.31 & 184739 & 51.46 \\
\hline 2 & 56.795 & 13612827 & 49.69 & 174266 & 48.54 \\
\hline
\end{tabular}

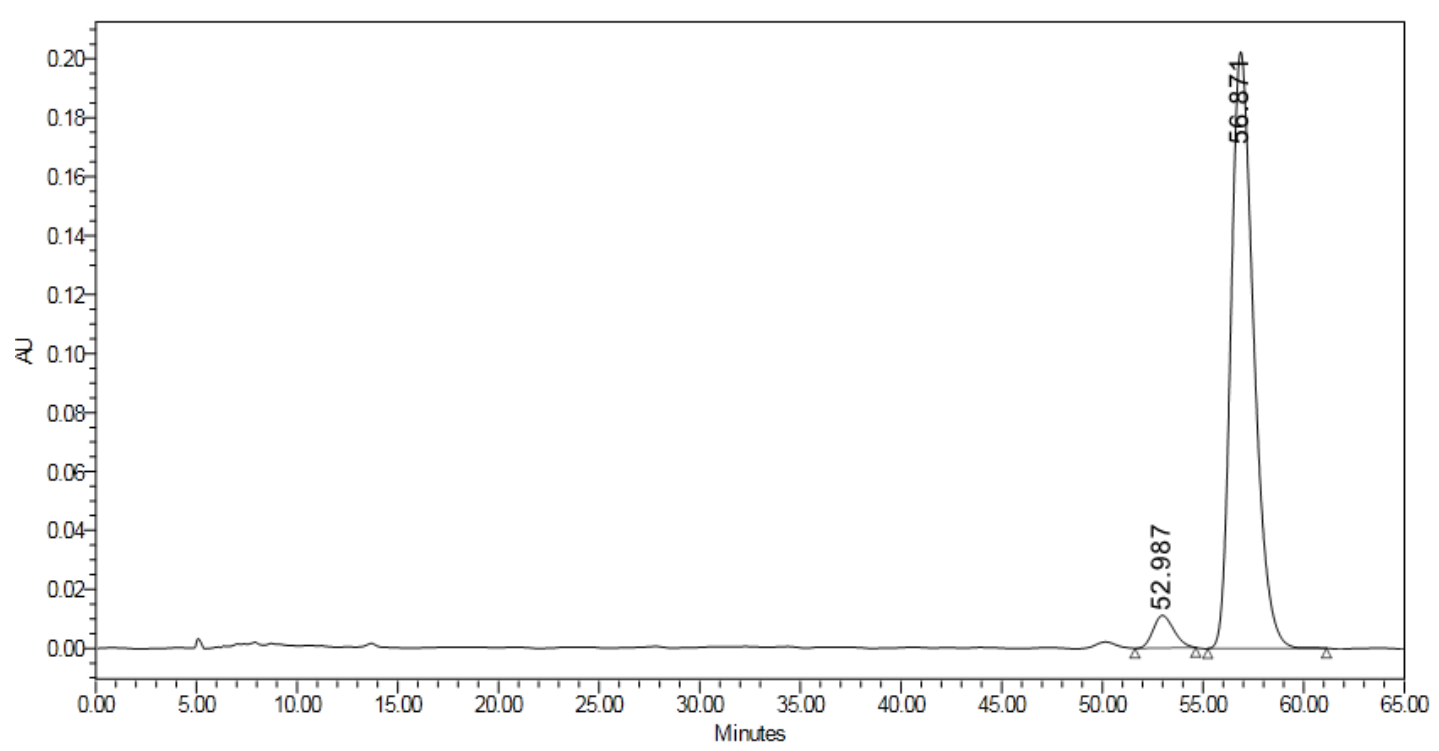

\begin{tabular}{|l|c|r|r|r|r|}
\hline & $\begin{array}{c}\mathrm{RT} \\
(\mathrm{min})\end{array}$ & $\begin{array}{c}\text { Area } \\
\left(\mu \mathrm{V}^{*} \mathrm{sec}\right)\end{array}$ & $\%$ Area & $\begin{array}{c}\text { Height } \\
(\mu \mathrm{V})\end{array}$ & $\begin{array}{c}\% \\
\text { Height }\end{array}$ \\
\hline 1 & 52.987 & 787519 & 4.72 & 11041 & 5.17 \\
\hline 2 & 56.871 & 15909380 & 95.28 & 202417 & 94.83 \\
\hline
\end{tabular}

